\title{
A Mild Photocatalytic Synthesis of Guanidine from Thiourea under Visible Light
}

Trin Saetan, ${ }^{\mathrm{a}}$ Mongkol Sukwattanasinitt, ${ }^{\mathrm{a}}$ and Sumrit Wacharasindhu ${ }^{\mathrm{a}, \mathrm{b} *}$

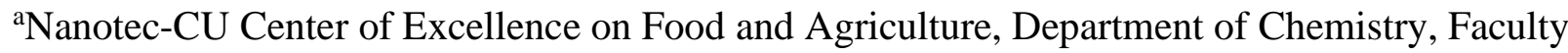
of Science, Chulalongkorn University Bangkok 10330, Thailand

${ }^{\mathrm{b}}$ Green Chemistry for Fine Chemical Productions STAR, Department of Chemistry, Faculty of Science, Chulalongkorn University Bangkok10330

E-mail: Sumrit.w@chula.ac.th 


\section{Table of Contents}

$\begin{array}{ll}\text { Title Page } & \text { S1 }\end{array}$

Material and method $\quad$ S3-S4

General procedure $\quad$ S4-S13

Copy of ${ }^{1} \mathrm{H} /{ }^{13} \mathrm{C}$ NMR of compound 2a-3j $\quad$ S14-S33

$\begin{array}{ll}\text { Copy of mass spectrum of compound } 5 & \text { S34 }\end{array}$

$\begin{array}{ll}\text { Copy of mass spectrum of compound } \mathbf{2 a - 3 j} & \text { S35-S53 }\end{array}$

$\begin{array}{ll}\text { Copy of SEM and EDX images } & \text { S54 }\end{array}$

$\begin{array}{ll}\text { References } & \text { S55 }\end{array}$ 


\section{Materials and methods}

Commercially available chemicals and solvents were used without further purification unless otherwise noted. High-resolution mass spectra (HRMS) were recorded using electron spray ionization (ESI) with a MicroTOF Bruker mass spectrometer. Analytical thin-layer chromatography (TLC) was performed with precoated Merck silica gel 60 F254 plates (thick layer, $0.25 \mathrm{~mm}$ ) and visualized at $254 \mathrm{~nm}$ using an ultraviolet lamp. Gel column chromatography was performed with Merck silica gel 60 (70-230 mesh). ${ }^{1} \mathrm{H}$ NMR and ${ }^{13} \mathrm{C}$ NMR spectra were obtained with a Varian Mercury 400 NMR or Bruker Avance 400 NMR or JEOL JNM-ECZ500R/S1 NMR spectrometers operating at 400 or $500 \mathrm{MHz}$ for ${ }^{1} \mathrm{H}$ and 100 or $125 \mathrm{MHz}$ for ${ }^{13} \mathrm{C}$ nuclei. Chemical shifts $(\delta)$ are reported in parts per million relative to the residual solvent signals and coupling constants $(J)$ are reported in hertz. 19W LED (Philips LED 19W Durable Brightness Daylight E27) and 42W CFL (Philips Tornado 42W E27 CFL) were used as the visible light source. Relative light intensity of 19W LED (blue) and 42W CFL (orange) were recorded by Ocean Optics USB2000 Fiber Optic Spectrometer.

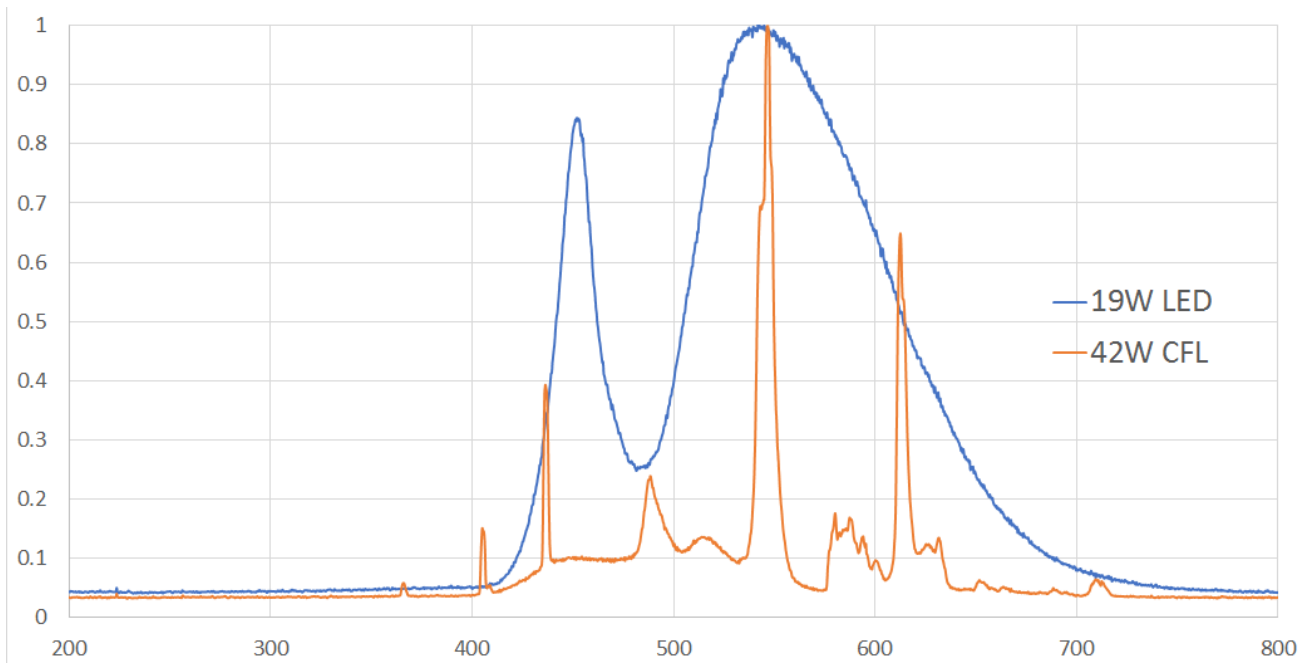

Figure S1. Relative light intensity of 19W LED (blue) and 42W CFL (orange) 


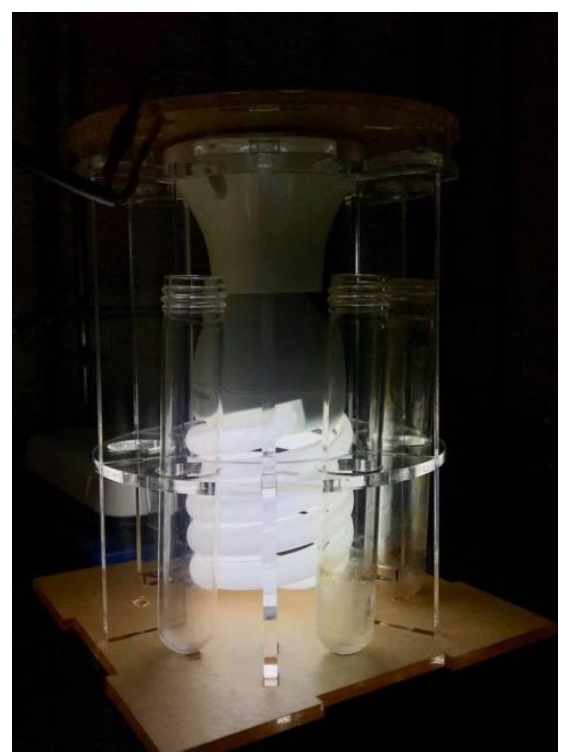

(a)

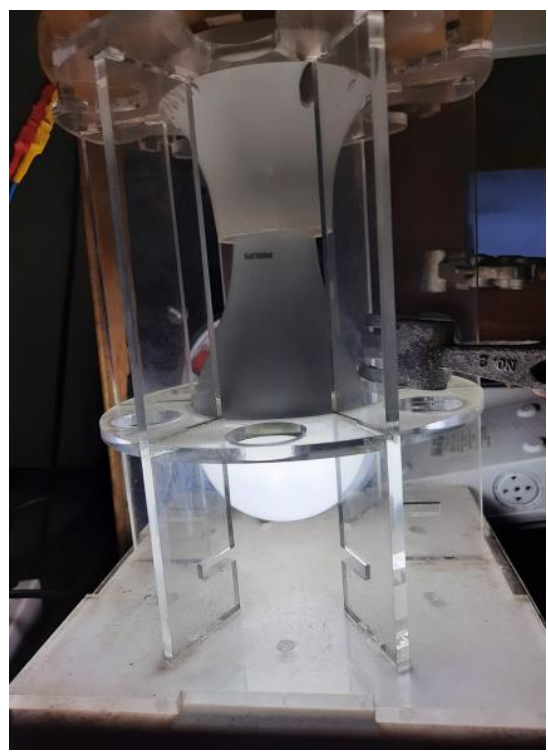

(b)

Figure S2. Photoreactor equipped with (a) 42W CFL light bulb and (b) 19W white LED

General procedure for substrate scope of amine in the reaction with 1,3-diphenylthiourea (Scheme 2)

A Pyrex glass tube equipped with a magnetic stirring bar was charged with $\mathrm{K}_{2} \mathrm{CO}_{3}(1.0 \mathrm{mmol})$ and $\mathrm{Ru}(\mathrm{bpy})_{3} \mathrm{Cl}_{2} \cdot 6 \mathrm{H}_{2} \mathrm{O}$ (1 mol\%). 1,3-diphenylthiourea (1a, $\left.0.5 \mathrm{mmol}\right)$, amine $(2.0 \mathrm{mmol})$, and $5 \mathrm{~mL}$ of ethanol: $\mathrm{H}_{2} \mathrm{O}$ (9:1) were added and reaction vessel was placed away from light source (19W LED) $4 \mathrm{~cm}$. The reaction mixture was stirred at room temperature for $24 \mathrm{~h}$ under $19 \mathrm{~W}$ white LED irradiation (Fig S2) the mixture was filtered, and the residue was purified by chromatography (hexane/ethyl acetate).

$N, N^{\prime}$-diphenylmorpholine-4-carboximidamide $(2 a)^{l}$ : According to the general procedure, the reaction was performed by using 1,3-diphenylthiourea (1a, $114 \mathrm{mg}, 0.5 \mathrm{mmol})$, morpholine (174 mg, $2.0 \mathrm{mmol}), \mathrm{Ru}(\mathrm{bpy})_{3} \mathrm{Cl}_{2} 6 \mathrm{H}_{2} \mathrm{O}(4 \mathrm{mg}, 0.005 \mathrm{mmol})$, and $\mathrm{K}_{2} \mathrm{CO}_{3}(138 \mathrm{mg}, 1.0 \mathrm{mmol})$ in $5 \mathrm{~mL}$ of EtOH: $\mathrm{H}_{2} \mathrm{O}(9: 1)$ for $24 \mathrm{~h}$ to afford $\mathbf{2 a}(126 \mathrm{mg}, 0.45 \mathrm{mmol}, 90 \%)$ as a yellow solid ${ }^{1} \mathrm{H}$ NMR 
$\left(400 \mathrm{MHz}, \mathrm{CDCl}_{3}\right) \delta 7.27(\mathrm{t}, J=7.6 \mathrm{~Hz}, 4 \mathrm{H}), 6.98(\mathrm{t}, J=13.6 \mathrm{~Hz}, 6 \mathrm{H}), 4.84(\mathrm{~s}, 1 \mathrm{H}), 3.70-3.68$ (m, 4H), $3.37-3.35(\mathrm{~m}, 4 \mathrm{H}) . ;{ }^{13} \mathrm{C} \mathrm{NMR}\left(100 \mathrm{MHz}, \mathrm{CDCl}_{3}\right) \delta$ 151.0, 129.4, 122.6, 120.6, 66.4, 47.1.; HRMS (ESI/Q-TOF) m/z:[M+H] ${ }^{+}$calcd for $\mathrm{C}_{17} \mathrm{H}_{20} \mathrm{~N}_{3} \mathrm{O} 282.1606$; found 282.1613

$N, N^{\prime}$-diphenylmorpholine-4-carboximidamide $(2 a)^{l}$ : According to the general procedure, the reaction was performed by using 1,3-diphenylthiourea (1a, $228 \mathrm{mg}, 1.0 \mathrm{mmol})$, morpholine (348 $\mathrm{mg}, 4.0 \mathrm{mmol}), \mathrm{Ru}(\mathrm{bpy}){ }_{3} \mathrm{Cl}_{2} \cdot 6 \mathrm{H}_{2} \mathrm{O}(8 \mathrm{mg}, 0.010 \mathrm{mmol})$, and $\mathrm{K}_{2} \mathrm{CO}_{3}(276 \mathrm{mg}, 2.0 \mathrm{mmol})$ in $10 \mathrm{~mL}$ of EtOH: $\mathrm{H}_{2} \mathrm{O}(9: 1)$ for $24 \mathrm{~h}$ to afford $\mathbf{2 a}(244 \mathrm{mg}, 0.87 \mathrm{mmol}, 87 \%)$ as a yellow solid ${ }^{1} \mathrm{H}$ NMR (400 $\left.\mathrm{MHz}, \mathrm{CDCl}_{3}\right) \delta 7.27(\mathrm{t}, J=7.6 \mathrm{~Hz}, 4 \mathrm{H}), 6.98(\mathrm{t}, J=13.6 \mathrm{~Hz}, 6 \mathrm{H}), 4.84(\mathrm{~s}, 1 \mathrm{H}), 3.70-3.68(\mathrm{~m}$, 4H), $3.37-3.35(\mathrm{~m}, 4 \mathrm{H}) . ;{ }^{13} \mathrm{C} \mathrm{NMR}\left(100 \mathrm{MHz}, \mathrm{CDCl}_{3}\right) \delta 151.0,129.4,122.6,120.6,66.4,47.1$.

1-butyl-2,3-diphenylguanidine $(\mathbf{2 b})^{2}$ : According to the general procedure, the reaction was performed by using 1,3-diphenylthiourea(1a, $114 \mathrm{mg}, 0.5 \mathrm{mmol})$, butylamine (146 mg, $2.0 \mathrm{mmol})$, $\mathrm{Ru}(\text { bpy })_{3} \mathrm{Cl}_{2} 6 \mathrm{H}_{2} \mathrm{O}(4 \mathrm{mg}, 0.005 \mathrm{mmol})$, and $\mathrm{K}_{2} \mathrm{CO}_{3}(138 \mathrm{mg}, 1.0 \mathrm{mmol})$ in $5 \mathrm{~mL}$ of EtOH: $\mathrm{H}_{2} \mathrm{O}(9: 1)$ for $24 \mathrm{~h}$ to afford $\mathbf{2 b}(109 \mathrm{mg}, 0.41 \mathrm{mmol}, 82 \%)$ as a yellow solid ${ }^{1} \mathrm{H}$ NMR (400 $\left.\mathrm{MHz}, \mathrm{CDCl}_{3}\right) \delta 7.32(\mathrm{t}, J=7.7 \mathrm{~Hz}, 4 \mathrm{H}), 7.06(\mathrm{t}, J=6.9 \mathrm{~Hz}, 6 \mathrm{H}), 4.68(\mathrm{~s}, 1 \mathrm{H}), 3.34(\mathrm{t}, J=7.2 \mathrm{~Hz}$, 2H), $1.60-1.53(\mathrm{~m}, 2 \mathrm{H}), 1.38(\mathrm{dq}, J=14.6,7.4 \mathrm{~Hz}, 2 \mathrm{H}), 0.95(\mathrm{t}, J=7.3 \mathrm{~Hz}, 3 \mathrm{H}) \cdot{ }^{13} \mathrm{C}$ NMR $(100$ $\left.\mathrm{MHz}, \mathrm{CDCl}_{3}\right) \delta 148.8,129.5,123.3,123.1,41.8,31.9,29.8,20.3,13.9 . ;$ HRMS (ESI/Q-TOF) $\mathrm{m} / \mathrm{z}:[\mathrm{M}+\mathrm{H}]^{+}$calcd for $\mathrm{C}_{17} \mathrm{H}_{22} \mathrm{~N}_{3} 268.6814$; found 268.1821

1-benzyl-2,3-diphenylguanidine $(2 c)^{3}$ : According to the general procedure, the reaction was performed by using 1,3-diphenylthiourea (1a, $114 \mathrm{mg}, 0.5 \mathrm{mmol})$, phenylmethanamine (214 $\mathrm{mg}$, $2.0 \mathrm{mmol}), \mathrm{Ru}(\mathrm{bpy}){ }_{3} \mathrm{Cl}_{2} 6 \mathrm{H}_{2} \mathrm{O}$ (4 mg, $\left.0.005 \mathrm{mmol}\right)$, and $\mathrm{K}_{2} \mathrm{CO}_{3}(138 \mathrm{mg}, 1.0 \mathrm{mmol})$ in $5 \mathrm{~mL}$ of EtOH: $\mathrm{H}_{2} \mathrm{O}(9: 1)$ for $24 \mathrm{~h}$ to afford $\mathbf{2 c}(114 \mathrm{mg}, 0.38 \mathrm{mmol}, 76 \%)$ as a yellow solid ${ }^{1} \mathrm{H}$ NMR (400 $\left.\mathrm{MHz}, \mathrm{CDCl}_{3}\right) \delta 7.36-7.28(\mathrm{~m}, 9 \mathrm{H}), 7.06(\mathrm{t}, J=8.8 \mathrm{~Hz}, 6 \mathrm{H}), 4.79(\mathrm{~s}, 1 \mathrm{H}), 4.54(\mathrm{~s}, 2 \mathrm{H}) .13^{\mathrm{C}} \mathrm{NMR}$ 
$\left(100 \mathrm{MHz}, \mathrm{CDCl}_{3}\right) \delta 148.5,139.0,129.5,128.7,127.7,127.4,123.4,123.1,46.0 . ;$ HRMS (ESI/QTOF) $\mathrm{m} / \mathrm{z}:[\mathrm{M}+\mathrm{H}]^{+}$calcd for $\mathrm{C}_{20} \mathrm{H}_{20} \mathrm{~N}_{3} 302.1657$; found 302.1665

1-cyclohexyl-2,3-diphenylguanidine $(\mathbf{2 d})^{3}$ : According to the general procedure, the reaction was performed by using 1,3-diphenylthiourea (1a, $114 \mathrm{mg}, 0.5 \mathrm{mmol})$, cyclohexylamine (198 $\mathrm{mg}, 2.0$ mmol), $\mathrm{Ru}(\text { bpy })_{3} \mathrm{Cl}_{2} \cdot 6 \mathrm{H}_{2} \mathrm{O}(20 \mathrm{mg}, 0.025 \mathrm{mmol})$, and $\mathrm{K}_{2} \mathrm{CO}_{3}(138 \mathrm{mg}, 1.0 \mathrm{mmol})$ in $5 \mathrm{~mL}$ of EtOH: $\mathrm{H}_{2} \mathrm{O}(9: 1)$ for $24 \mathrm{~h}$ to afford $\mathbf{2 d}(117 \mathrm{mg}, 0.40 \mathrm{mmol}, 80 \%)$ as a yellow solid ${ }^{1} \mathrm{H}$ NMR (400 $\left.\mathrm{MHz}, \mathrm{CDCl}_{3}\right) \delta 7.30(\mathrm{t}, \mathrm{J}=7.7 \mathrm{~Hz}, 4 \mathrm{H}), 7.05(\mathrm{t}, \mathrm{J}=7.2 \mathrm{~Hz}, 6 \mathrm{H}), 3.74(\mathrm{t}, \mathrm{J}=10.2 \mathrm{~Hz}, 1 \mathrm{H}), 2.08$ $(\mathrm{d}, \mathrm{J}=12.0 \mathrm{~Hz}, 2 \mathrm{H}), 1.70(\mathrm{dd}, \mathrm{J}=9.6,3.7 \mathrm{~Hz}, 2 \mathrm{H}), 1.61(\mathrm{dd}, \mathrm{J}=9.0,3.7 \mathrm{~Hz}, 2 \mathrm{H}), 1.44-1.31(\mathrm{~m}$, 2H), $1.23-1.08(\mathrm{~m}, 2 \mathrm{H}) . ; 13^{\mathrm{C}} \mathrm{NMR}\left(100 \mathrm{MHz}, \mathrm{CDCl}_{3}\right) \delta$ 148.1, 129.5, 123.3, 123.0, 50.2, 33.5,29.8, 25.8, 25.0.; HRMS (ESI/Q-TOF) m/z:[M+H] $]^{+}$calcd for $\mathrm{C}_{19} \mathrm{H}_{24} \mathrm{~N}_{3}$ 294.1970; found 294.1976

ethyl 3-(2,3-diphenylguanidino) propanoate (2e): According to the general procedure, the reaction was performed by using 1,3-diphenylthiourea (1a, $114 \mathrm{mg}, 0.5 \mathrm{mmol})$, ethyl 3-aminopropanoate (234 mg, $2.0 \mathrm{mmol}), \mathrm{Ru}(\mathrm{bpy})_{3} \mathrm{Cl}_{2} \cdot 6 \mathrm{H}_{2} \mathrm{O}$ (4 mg, $\left.0.005 \mathrm{mmol}\right)$, and $\mathrm{K}_{2} \mathrm{CO}_{3}(138 \mathrm{mg}, 1.0 \mathrm{mmol})$ in $5 \mathrm{~mL}$ of EtOH: $\mathrm{H}_{2} \mathrm{O}(9: 1)$ for $24 \mathrm{~h}$ to afford $2 \mathbf{e}(116 \mathrm{mg}, 0.37 \mathrm{mmol}, 75 \%)$ as a yellow solid ${ }^{1} \mathrm{H}$ NMR $\left(400 \mathrm{MHz}, \mathrm{CDCl}_{3}\right) \delta 7.25(\mathrm{dd}, \mathrm{J}=11.0,4.3 \mathrm{~Hz}, 4 \mathrm{H}), 7.05-6.85(\mathrm{~m}, 6 \mathrm{H}), 3.47-3.36(\mathrm{~m}, 4 \mathrm{H})$, $3.36-3.24(\mathrm{~m}, 4 \mathrm{H}), 1.45(\mathrm{~s}, 9 \mathrm{H}) .{ }^{13} \mathrm{C}$ NMR $\left(100 \mathrm{MHz}, \mathrm{CDCl}_{3}\right) \delta 154.8,151.0,129.4,122.7,120.6$, 80.0, 46.5, 43.2, 28.4. HRMS (ESI/Q-TOF) m/z:[M+H] calcd for $\mathrm{C}_{18} \mathrm{H}_{22} \mathrm{~N}_{3} \mathrm{O}_{2} 312.1712$; found 312.1715

1-(2-hydroxyethyl)-2,3-diphenylguanidine $(2 f)^{4}$ : According to the general procedure, the reaction was performed by using 1,3-diphenylthiourea(1a, $114 \mathrm{mg}, 0.5 \mathrm{mmol})$, 2-aminoethanol (122 $\mathrm{mg}$, $2.0 \mathrm{mmol}$ ), $\mathrm{Ru}(\mathrm{bpy})_{3} \mathrm{Cl}_{2} 6 \mathrm{H}_{2} \mathrm{O}(4 \mathrm{mg}, 0.005 \mathrm{mmol})$, and $\mathrm{K}_{2} \mathrm{CO}_{3}(138 \mathrm{mg}, 1.0 \mathrm{mmol})$ in $5 \mathrm{~mL}$ of EtOH: $\mathrm{H}_{2} \mathrm{O}(9: 1)$ for $24 \mathrm{~h}$ to afford $\mathbf{2 f}(102 \mathrm{mg}, 0.40 \mathrm{mmol}, 80 \%)$ as a yellow solid ${ }^{1} \mathrm{H}$ NMR (400 
$\left.\mathrm{MHz} \mathrm{CDCl}_{3}\right) \delta 7.29(\mathrm{~d}, J=5.0 \mathrm{~Hz}, 4 \mathrm{H}), 7.08(\mathrm{~d}, J=7.7 \mathrm{~Hz}, 4 \mathrm{H}), 7.03(\mathrm{t}, J=7.4 \mathrm{~Hz}, 2 \mathrm{H}), 3.60(\mathrm{t}$, $J=4.6 \mathrm{~Hz}, 2 \mathrm{H}), 3.53(\mathrm{~d}, J=4.8 \mathrm{~Hz}, 2 \mathrm{H}) \cdot{ }^{13} \mathrm{C} \mathrm{NMR}\left(100 \mathrm{MHz}, \mathrm{CDCl}_{3}\right) \delta 129.4,123.4,122.6$, $72.6,59.0,42.3,32.1,29.8,29.5,22.8,14.2$.

1,1-diethyl-2,3-diphenylguanidine $(\mathbf{2 g})^{5}$ : According to the general procedure, the reaction was performed by using 1,3-diphenylthiourea (1a, $114 \mathrm{mg}, 0.5 \mathrm{mmol})$, diethylamine (146 mg, 2.0 mmol), $\mathrm{Ru}(\mathrm{bpy}){ }_{3} \mathrm{Cl}_{2} \cdot 6 \mathrm{H}_{2} \mathrm{O}(4 \mathrm{mg}, 0.005 \mathrm{mmol})$, and $\mathrm{K}_{2} \mathrm{CO}_{3}(138 \mathrm{mg}, 1.0 \mathrm{mmol})$ in $5 \mathrm{~mL}$ of EtOH: $\mathrm{H}_{2} \mathrm{O}(9: 1)$ for 3 days to afford $\mathbf{2 g}(109 \mathrm{mg}, 0.41 \mathrm{mmol}, 82 \%)$ as a yellow solid ${ }^{1} \mathrm{H}$ NMR (400 $\mathrm{MHz}, \mathrm{CDCl} 3) \delta 7.22(\mathrm{t}, \mathrm{J}=7.8 \mathrm{~Hz}, 4 \mathrm{H}), 6.94(\mathrm{t}, \mathrm{J}=7.4 \mathrm{~Hz}, 2 \mathrm{H}), 6.89(\mathrm{~d}, \mathrm{~J}=7.7 \mathrm{~Hz}, 4 \mathrm{H}), 3.34(\mathrm{q}$, $\mathrm{J}=7.1 \mathrm{~Hz}, 4 \mathrm{H}), 1.17(\mathrm{t}, \mathrm{J}=7.1 \mathrm{~Hz}, 6 \mathrm{H}) .{ }^{13} \mathrm{C} \mathrm{NMR}\left(100 \mathrm{MHz}, \mathrm{CDCl}_{3}\right) \delta 129.3,122.2,42.3,13.0$; HRMS (ESI/Q-TOF) m/z:[M+H] $]^{+}$calcd for $\mathrm{C}_{17} \mathrm{H}_{22} \mathrm{~N}_{3} 268.1808$; found 268.1797

2-methyl-N,N'-diphenylpiperidine-1-carboximidamide (2h): According to the general procedure, the reaction was performed by using 1,3-diphenylthiourea (1a, $114 \mathrm{mg}, 0.5 \mathrm{mmol})$, 2methylpiperidine (198 mg, $2.0 \mathrm{mmol}), \mathrm{Ru}(\mathrm{bpy}){ }_{3} \mathrm{Cl}_{2} \cdot 6 \mathrm{H}_{2} \mathrm{O}(4 \mathrm{mg}, 0.005 \mathrm{mmol})$, and $\mathrm{K}_{2} \mathrm{CO}_{3}(138$ $\mathrm{mg}, 1.0 \mathrm{mmol})$ in $5 \mathrm{~mL}$ of $\mathrm{EtOH}: \mathrm{H}_{2} \mathrm{O}(9: 1)$ for $24 \mathrm{~h}$ to afford $\mathbf{2 h}(131 \mathrm{mg}, 0.45 \mathrm{mmol}, 90 \%)$ as a yellow solid ${ }^{1} \mathrm{H}$ NMR $\left(400 \mathrm{MHz}, \mathrm{CDCl}_{3}\right) \delta 7.25(\mathrm{t}, J=7.5 \mathrm{~Hz}, 4 \mathrm{H}), 6.96(\mathrm{dd}, J=16.5,7.7 \mathrm{~Hz}$, $6 \mathrm{H}), 4.17(\mathrm{~d}, J=5.4 \mathrm{~Hz}, 1 \mathrm{H}), 3.70(\mathrm{~d}, J=12.9 \mathrm{~Hz}, 1 \mathrm{H}), 3.04(\mathrm{t}, J=12.0 \mathrm{~Hz}, 1 \mathrm{H}), 1.86-1.49(\mathrm{~m}$, $6 \mathrm{H}), 1.23(\mathrm{~d}, J=6.8 \mathrm{~Hz}, 3 \mathrm{H}) ;{ }^{13} \mathrm{C} \mathrm{NMR}\left(100 \mathrm{MHz}, \mathrm{CDCl}_{3}\right) \delta 151.0,129.3,122.2,120.4,49.0$, 41.6, 29.9, 29.8, 25.6, 19.2, 14.7. ; HRMS (ESI/Q-TOF) m/z: $[\mathrm{M}+\mathrm{H}]^{+}$calcd for $\mathrm{C}_{19} \mathrm{H}_{24} \mathrm{~N}_{3} 294.1970$; found 294.1966

$N, N^{\prime}$-diphenylpyrrolidine-1-carboximidamide $(2 \boldsymbol{i})^{6}$ : According to the general procedure, the reaction was performed by using 1,3-diphenylthiourea (1a, $114 \mathrm{mg}, 0.5 \mathrm{mmol})$, pyrrolidine (142 mg, $2.0 \mathrm{mmol}), \mathrm{Ru}(\mathrm{bpy})_{3} \mathrm{Cl}_{2} 6 \mathrm{H}_{2} \mathrm{O}$ (4 mg, $\left.0.005 \mathrm{mmol}\right)$, and $\mathrm{K}_{2} \mathrm{CO}_{3}(138 \mathrm{mg}, 1.0 \mathrm{mmol})$ in $5 \mathrm{~mL}$ of $\mathrm{EtOH}: \mathrm{H}_{2} \mathrm{O}(9: 1)$ for $24 \mathrm{~h}$ to afford $2 \mathbf{i}(87 \mathrm{mg}, 0.33 \mathrm{mmol}, 66 \%)$ as a yellow solid ${ }^{1} \mathrm{H}$ NMR (500 
$\left.\mathrm{MHz}, \mathrm{CDCl}_{3}\right) \delta 7.24-7.20(\mathrm{~m}, 4 \mathrm{H}), 6.93(\mathrm{ddd}, J=9.3,7.8,0.9 \mathrm{~Hz}, 6 \mathrm{H}), 3.36(\mathrm{t}, J=6.6 \mathrm{~Hz}, 4 \mathrm{H})$, $1.92-1.79(\mathrm{~m}, 4 \mathrm{H}) . ; 13^{\mathrm{C}} \mathrm{NMR}\left(125 \mathrm{MHz}, \mathrm{CDCl}_{3}\right) \delta 149.5,129.3,122.1,121.0,47.8,25.4$; HRMS (ESI/Q-TOF) m/z:[M+H] $]^{+}$calcd for $\mathrm{C}_{17} \mathrm{H}_{20} \mathrm{~N}_{3} 266.1657$; found 266.1661

$N, N^{\prime}$-diphenylazepane-1-carboximidamide (2j): According to the general procedure, the reaction was performed by using 1,3-diphenylthiourea (1a, $114 \mathrm{mg}, 0.5 \mathrm{mmol})$, azepane (198 $\mathrm{mg}, 2.0$ mmol), $\mathrm{Ru}(\mathrm{bpy})_{3} \mathrm{Cl}_{2} \cdot 6 \mathrm{H}_{2} \mathrm{O}(4 \mathrm{mg}, 0.005 \mathrm{mmol})$, and $\mathrm{K}_{2} \mathrm{CO}_{3}(138 \mathrm{mg}, 1.0 \mathrm{mmol})$ in $5 \mathrm{~mL}$ of EtOH: $\mathrm{H}_{2} \mathrm{O}(9: 1)$ for $24 \mathrm{~h}$ to afford $\mathbf{2 j}$ (54 $\mathrm{mg}, 0.18 \mathrm{mmol}, 37 \%$ ) as a yellow solid ${ }^{1} \mathrm{H}$ NMR (500 $\left.\mathrm{MHz}, \mathrm{CDCl}_{3}\right) \delta 7.23-7.19(\mathrm{~m}, 4 \mathrm{H}), 6.95-6.93(\mathrm{~m}, 2 \mathrm{H}), 6.86(\mathrm{dd}, J=8.5,1.1 \mathrm{~Hz}, 4 \mathrm{H}), 3.46-$ $3.44(\mathrm{~m}, 4 \mathrm{H}), 1.74-1.73(\mathrm{~m}, 4 \mathrm{H}), 1.61(\mathrm{dt}, J=7.1,2.7 \mathrm{~Hz}, 4 \mathrm{H}) . ;{ }^{13} \mathrm{C} \mathrm{NMR}\left(125 \mathrm{MHz}, \mathrm{CDCl}_{3}\right) \delta$ 151.0, 129.3, 122.0, 120.7, 48.9, 28.2, 27.9.; HRMS (ESI/Q-TOF) m/z:[M+H] $]^{+}$calcd for $\mathrm{C}_{19} \mathrm{H}_{24} \mathrm{~N}_{3}$ 294.1970; found 294.1974

tert-butyl-4-(N,N'-diphenylcarbamimidoyl)piperazine-1-carboxylate $(\mathbf{2 k})$ : According to the general procedure, the reaction was performed by using 1,3-diphenylthiourea (1a, $114 \mathrm{mg}, 0.5$ mmol), tert-butyl piperazine-1-carboxylate (372 mg, $2.0 \mathrm{mmol}$ ), $\mathrm{Ru}(\mathrm{bpy})_{3} \mathrm{Cl}_{2} \cdot 6 \mathrm{H}_{2} \mathrm{O}$ (20 mg, 0.025 $\mathrm{mmol}$ ), and $\mathrm{K}_{2} \mathrm{CO}_{3}(138 \mathrm{mg}, 1.0 \mathrm{mmol})$ in $5 \mathrm{~mL}$ of $\mathrm{EtOH}: \mathrm{H}_{2} \mathrm{O}(9: 1)$ for $24 \mathrm{~h}$ to afford $2 \mathbf{k}$ (54 mg, $0.18 \mathrm{mmol}, 37 \%)$ as a yellow solid ${ }^{1} \mathrm{H} \mathrm{NMR}\left(400 \mathrm{MHz}, \mathrm{CDCl}_{3}\right) \delta 7.25(\mathrm{dd}, J=11.0,4.3 \mathrm{~Hz}, 4 \mathrm{H})$, $7.00-6.92(\mathrm{~m}, 6 \mathrm{H}), 3.42-3.40(\mathrm{~m}, 4 \mathrm{H}), 3.31-3.29(\mathrm{~m}, 4 \mathrm{H}), 1.45(\mathrm{~s}, 9 \mathrm{H}) ;{ }^{13} \mathrm{C}$ NMR $(100 \mathrm{MHz}$, $\left.\mathrm{CDCl}_{3}\right) \delta 154.8,151.0,129.4,122.7,120.6,80.0,46.5,28.4 . ;$ HRMS (ESI/Q-TOF) m/z:[M+H] ${ }^{+}$ calcd for $\mathrm{C}_{22} \mathrm{H}_{29} \mathrm{~N}_{4} \mathrm{O}_{2} 381.2291$; found 381.2291

General procedure for Substrate scope of thiourea in the reaction with morpholine (Scheme 3) 
A pyrex glass tube equipped with a magnetic stirring bar was charged with $\mathrm{K}_{2} \mathrm{CO}_{3}(1.0 \mathrm{mmol})$ and $\mathrm{Ru}(\mathrm{bpy})_{3} \mathrm{Cl}_{2} .6 \mathrm{H}_{2} \mathrm{O}(1 \mathrm{~mol} \%)$. Thiourea derivative $(0.5 \mathrm{mmol})$, morpholine $(2.0 \mathrm{mmol})$, and $5 \mathrm{~mL}$ of ethanol: $\mathrm{H}_{2} \mathrm{O}(9: 1)$ were added and placed in the $19 \mathrm{~W}$ white LED light reactor. The reaction mixture was stirred at room temperature for $24 \mathrm{~h}$ under 19W white LED irradiation. the mixture was filtered, and the residue was purified by chromatography (hexane/ethyl acetate).

$N^{\prime}$-phenyl-N-(o-tolyl)morpholine-4-carboximidamide $(\mathbf{3} \boldsymbol{b})$ : According to the general procedure, the reaction was performed by using 1-phenyl-3-(o-tolyl)thiourea $(\mathbf{1 b}, 121 \mathrm{mg}, 0.5 \mathrm{mmol})$, morpholine (174 mg, $2.0 \mathrm{mmol}$ ), $\mathrm{Ru}(\mathrm{bpy}){ }_{3} \mathrm{Cl}_{2} \cdot 6 \mathrm{H}_{2} \mathrm{O}$ (4 mg, $0.005 \mathrm{mmol}$ ), and $\mathrm{K}_{2} \mathrm{CO}_{3}(138 \mathrm{mg}, 1.0$ mmol) in $5 \mathrm{~mL}$ of EtOH: $\mathrm{H}_{2} \mathrm{O}(9: 1)$ for $24 \mathrm{~h}$ to afford $\mathbf{3 b}(121 \mathrm{mg}, 0.41 \mathrm{mmol}, 82 \%)$ as a yellow solid ${ }^{1} \mathrm{H}$ NMR (400 MHz, $\left.\mathrm{CDCl}_{3}\right) \delta 7.29(\mathrm{~s}, 2 \mathrm{H}), 7.17(\mathrm{~d}, J=6.4 \mathrm{~Hz}, 2 \mathrm{H}), 7.03(\mathrm{~s}, 5 \mathrm{H}), 3.66(\mathrm{~s}$, 4H), $3.33(\mathrm{~s}, 4 \mathrm{H}), 2.17(\mathrm{~s}, 3 \mathrm{H}) ;{ }^{13} \mathrm{C} \mathrm{NMR}\left(100 \mathrm{MHz}, \mathrm{CDCl}_{3}\right) \delta 130.7,129.3,126.9,122.9,122.6$ 120.9, 66.4, 47.2, 29.7, 29.4.; HRMS (ESI/Q-TOF) m/z:[M+H] $]^{+}$calcd for $\mathrm{C}_{18} \mathrm{H}_{22} \mathrm{~N}_{3} \mathrm{O}$ 296.1763; found 296.1768

$N^{\prime}$-phenyl-N-(p-tolyl)morpholine-4-carboximidamide (3c): According to the general procedure, the reaction was performed by using 1-phenyl-3-(p-tolyl)thiourea $(\mathbf{1 c}, 121 \mathrm{mg}, 0.5 \mathrm{mmol})$, morpholine (174 mg, $2.0 \mathrm{mmol}$ ), $\mathrm{Ru}(\mathrm{bpy}){ }_{3} \mathrm{Cl}_{2} \cdot 6 \mathrm{H}_{2} \mathrm{O}$ (4 mg, $0.005 \mathrm{mmol}$ ), and $\mathrm{K}_{2} \mathrm{CO}_{3}(138 \mathrm{mg}, 1.0$ mmol) in $5 \mathrm{~mL}$ of EtOH: $\mathrm{H}_{2} \mathrm{O}(9: 1)$ for $24 \mathrm{~h}$ to afford $3 \mathrm{c}(126 \mathrm{mg}, 0.43 \mathrm{mmol}, 86 \%)$ as a yellow solid ${ }^{1} \mathrm{H}$ NMR $\left(500 \mathrm{MHz}, \mathrm{CDCl}_{3}\right) \delta 7.50(\mathrm{~d}, J=8.6 \mathrm{~Hz}, 2 \mathrm{H}), 7.24(\mathrm{t}, J=7.9 \mathrm{~Hz}, 2 \mathrm{H}), 6.98(\mathrm{t}, J=$ $7.4 \mathrm{~Hz}, 1 \mathrm{H}), 6.92(\mathrm{~d}, J=6.6 \mathrm{~Hz}, 2 \mathrm{H}), 6.66(\mathrm{~d}, J=7.5 \mathrm{~Hz}, 2 \mathrm{H}), 3.66-3.64(\mathrm{~m}, 4 \mathrm{H}), 3.31-3.30$ (m, 4H), 1.24 (s, 3H).; ${ }^{13} \mathrm{C}$ NMR (125 MHz, $\left.\mathrm{CDCl}_{3}\right) \delta$ 150.9, 138.3, 129.6, 122.8, 66.4, 47.1, 29.8, 29.5.; HRMS (ESI/Q-TOF) m/z:[M+H] ${ }^{+}$calcd for $\mathrm{C}_{18} \mathrm{H}_{22} \mathrm{~N}_{3} \mathrm{O} 296.1763$; found 268.2018

$N^{\prime}-(4-m e t h o x y p h e n y l)-N$-phenylmorpholine-4-carboximidamide $(3 \boldsymbol{d})^{7}$ : According to the general procedure, the reaction was performed by using 1-(4-methoxyphenyl)-3-phenylthiourea (1d, 129 
mg, $0.5 \mathrm{mmol}$ ), morpholine (174 mg, $2.0 \mathrm{mmol}), \mathrm{Ru}(\mathrm{bpy}){ }_{3} \mathrm{Cl}_{2} \cdot 6 \mathrm{H}_{2} \mathrm{O}(20 \mathrm{mg}, 0.025 \mathrm{mmol})$, and $\mathrm{K}_{2} \mathrm{CO}_{3}(138 \mathrm{mg}, 1.0 \mathrm{mmol})$ in $5 \mathrm{~mL}$ of $\mathrm{EtOH}: \mathrm{H}_{2} \mathrm{O}(9: 1)$ for $24 \mathrm{~h}$ to afford 3d $(135 \mathrm{mg}, 0.43 \mathrm{mmol}$, $87 \%$ ) as a yellow solid ${ }^{1} \mathrm{H} \mathrm{NMR}\left(400 \mathrm{MHz}, \mathrm{CDCl}_{3}\right) \delta 7.28(\mathrm{dd}, J=10.4,5.2 \mathrm{~Hz}, 2 \mathrm{H}), 6.99(\mathrm{dd}, J$ $=15.3,7.6 \mathrm{~Hz}, 3 \mathrm{H}), 6.91(\mathrm{~d}, J=8.6 \mathrm{~Hz}, 2 \mathrm{H}), 6.82(\mathrm{~d}, J=8.8 \mathrm{~Hz}, 2 \mathrm{H}), 3.78(\mathrm{~s}, 3 \mathrm{H}), 3.68-3.66$ (m, 4H), 3.35 - $3.32(\mathrm{~m}, 4 \mathrm{H}) . ;{ }^{13} \mathrm{C} \mathrm{NMR}\left(100 \mathrm{MHz}, \mathrm{CDCl}_{3}\right) \delta 130.8,129.4,126.9,123.0,122.6$ 121.0, 66.4, 47.3. ; HRMS (ESI/Q-TOF) m/z:[M+H] ${ }^{+}$calcd for $\mathrm{C}_{18} \mathrm{H}_{22} \mathrm{~N}_{3} \mathrm{O}_{2}$ 312.1712; found 312.1765

$N^{\prime}$-(4-bromophenyl)-N-phenylmorpholine-4-carboximidamide (3e): According to the general procedure, the reaction was performed by using 1-(4-bromophenyl)-3-phenylthiourea (1e, $154 \mathrm{mg}$, $0.5 \mathrm{mmol}$ ), morpholine (174 mg, $2.0 \mathrm{mmol}), \mathrm{Ru}(\mathrm{bpy}){ }_{3} \mathrm{Cl}_{2} \cdot 6 \mathrm{H}_{2} \mathrm{O}(20 \mathrm{mg}, 0.025 \mathrm{mmol})$, and $\mathrm{K}_{2} \mathrm{CO}_{3}$ (138 mg, $1.0 \mathrm{mmol})$ in $5 \mathrm{~mL}$ of EtOH: $\mathrm{H}_{2} \mathrm{O}(9: 1)$ for $24 \mathrm{~h}$ to afford $\mathbf{3 e}(137 \mathrm{mg}, 0.38 \mathrm{mmol}, 76 \%)$ as a yellow solid ${ }^{1} \mathrm{H} \mathrm{NMR}\left(400 \mathrm{MHz}, \mathrm{CDCl}_{3}\right) \delta 7.35(\mathrm{~d}, J=7.4 \mathrm{~Hz}, 2 \mathrm{H}), 7.28(\mathrm{t}, J=7.2 \mathrm{~Hz}, 2 \mathrm{H})$, $7.03-6.95(\mathrm{~m}, 3 \mathrm{H}), 6.81(\mathrm{~d}, J=7.7 \mathrm{~Hz}, 2 \mathrm{H}), 3.68(\mathrm{~s}, 4 \mathrm{H}), 3.34(\mathrm{~s}, 4 \mathrm{H}) ;{ }^{13} \mathrm{C} \mathrm{NMR}(100 \mathrm{MHz}$, $\left.\mathrm{CDCl}_{3}\right) \delta 150.9,132.3,129.5,122.8,66.3,47.0$. ; HRMS (ESI/Q-TOF) m/z:[M+H] $]^{+}$calcd for $\mathrm{C}_{17} \mathrm{H}_{19} \mathrm{BrN}_{3} \mathrm{O} 360.0711$; found 360.0715

$N^{\prime}$-(4-iodophenyl)-N-phenylmorpholine-4-carboximidamide (3f): According to the general procedure, the reaction was performed by using 1-(4-iodophenyl)-3-phenylthiourea (1f, $177 \mathrm{mg}$, $0.5 \mathrm{mmol})$, morpholine (174 mg, $2.0 \mathrm{mmol}), \mathrm{Ru}(\mathrm{bpy})_{3} \mathrm{Cl}_{2} \cdot 6 \mathrm{H}_{2} \mathrm{O}(4 \mathrm{mg}, 0.005 \mathrm{mmol})$, and $\mathrm{K}_{2} \mathrm{CO}_{3}$ (138 mg, $1.0 \mathrm{mmol})$ in $5 \mathrm{~mL}$ of $\mathrm{EtOH}: \mathrm{H}_{2} \mathrm{O}(9: 1)$ for $24 \mathrm{~h}$ to afford $\mathbf{3 f}(154 \mathrm{mg}, 0.38 \mathrm{mmol}, 76 \%)$ as a yellow solid ${ }^{1} \mathrm{H}$ NMR $\left(400 \mathrm{MHz}, \mathrm{CDCl}_{3}\right) \delta 8.21-8.20(\mathrm{~m}, 1 \mathrm{H}), 7.54(\mathrm{ddd}, J=8.3,7.3,1.1$ $\mathrm{Hz}, 1 \mathrm{H}), 7.27(\mathrm{t}, J=7.6 \mathrm{~Hz}, 2 \mathrm{H}), 7.14(\mathrm{~d}, J=7.8 \mathrm{~Hz}, 2 \mathrm{H}), 6.99(\mathrm{dd}, J=12.3,4.9 \mathrm{~Hz}, 2 \mathrm{H}), 6.82-$ $6.79(\mathrm{~m}, 1 \mathrm{H}), 3.69-3.67(\mathrm{~m}, 4 \mathrm{H}), 3.45-3.43(\mathrm{~m}, 4 \mathrm{H}) . ;{ }^{13} \mathrm{C} \mathrm{NMR}\left(100 \mathrm{MHz}, \mathrm{CDCl}_{3}\right) \delta 150.8$ 
138.2, 129.5, 122.8, 66.3, 47.0.; HRMS (ESI/Q-TOF) m/z:[M+H] $]^{+}$calcd for $\mathrm{C}_{17} \mathrm{H}_{19} \mathrm{IN}_{3} \mathrm{O}$ 408.0573; found 408.0579

$N$-phenyl-N'-(4-(trifluoromethyl)phenyl)morpholine-4-carboximidamide (3g): According to the general procedure, the reaction was performed by using 1-phenyl-3-(4(trifluoromethyl)phenyl)thiourea $(\mathbf{1 g}, 148 \mathrm{mg}, 0.5 \mathrm{mmol})$, morpholine (174 mg, $2.0 \mathrm{mmol})$, $\mathrm{Ru}(\mathrm{bpy})_{3} \mathrm{Cl}_{2} \cdot 6 \mathrm{H}_{2} \mathrm{O}(4 \mathrm{mg}, 0.005 \mathrm{mmol})$, and $\mathrm{K}_{2} \mathrm{CO}_{3}(138 \mathrm{mg}, 1.0 \mathrm{mmol})$ in $5 \mathrm{~mL}$ of EtOH: $\mathrm{H}_{2} \mathrm{O}(9: 1)$ for $24 \mathrm{~h}$ to afford $\mathbf{3 g}(89 \mathrm{mg}, 0.26 \mathrm{mmol}, 51 \%)$ as a yellow solid ${ }^{1} \mathrm{H}$ NMR (500 $\left.\mathrm{MHz}, \mathrm{CDCl}_{3}\right) \delta=7.46(\mathrm{~d}, \mathrm{~J}=8.4,2 \mathrm{H}), 7.25-7.24(\mathrm{~m}, 2 \mathrm{H}), 7.01-6.93(\mathrm{~m}, 5 \mathrm{H}), 3.69-3.67(\mathrm{~m}$, 4H), $3.36-3.34(\mathrm{~m}, 4 \mathrm{H}) . ;{ }^{13} \mathrm{C} \mathrm{NMR}\left(125 \mathrm{MHz}, \mathrm{CDCl}_{3}\right) \delta 150.8,129.6,126.7,126.6,123.2,66.3$, 47.2. ; HRMS (ESI/Q-TOF) m/z:[M+H] $]^{+}$calcd for $\mathrm{C}_{18} \mathrm{H}_{19} \mathrm{~F}_{3} \mathrm{~N}_{3} \mathrm{O} 350.1480$; found 350.1486

$N^{\prime}$-(3-chloro-4-(trifluoromethyl)phenyl)-N-phenylmorpholine-4-carboximidamide

$(3 h):$ According to the general procedure, the reaction was performed by using 1-(3-chloro-4(trifluoromethyl)phenyl)-3-phenylthiourea (1h, $165 \mathrm{mg}, 0.5 \mathrm{mmol}$ ), morpholine (174 mg, 2.0 mmol), $\mathrm{Ru}(\mathrm{bpy})_{3} \mathrm{Cl}_{2} 6 \mathrm{H}_{2} \mathrm{O}(4 \mathrm{mg}, 0.005 \mathrm{mmol})$, and $\mathrm{K}_{2} \mathrm{CO}_{3}(138 \mathrm{mg}, 1.0 \mathrm{mmol})$ in $5 \mathrm{~mL}$ of EtOH: $\mathrm{H}_{2} \mathrm{O}(9: 1)$ for $24 \mathrm{~h}$ to afford $\mathbf{3 h}(46 \mathrm{mg}, 0.12 \mathrm{mmol}, 24 \%)$ as a yellow solid ${ }^{1} \mathrm{H}$ NMR (500 $\left.\mathrm{MHz}, \mathrm{CDCl}_{3}\right) \delta 7.26-7.23(\mathrm{~m}, 4 \mathrm{H}), 6.97(\mathrm{dd}, J=15.3,7.8 \mathrm{~Hz}, 3 \mathrm{H}), 3.67-3.65(\mathrm{~m}, 4 \mathrm{H}), 3.34-$ $3.32(\mathrm{~m}, 4 \mathrm{H}) . ;{ }^{13} \mathrm{C}$ NMR $\left(125 \mathrm{MHz}, \mathrm{CDCl}_{3}\right) \delta 151.3,129.5,122.9,66.4,47.2 . ;$ HRMS (ESI/QTOF) m/z:[M+H] $]^{+}$calcd for $\mathrm{C}_{18} \mathrm{H}_{18} \mathrm{ClF}_{3} \mathrm{~N}_{3} \mathrm{O} 384.0190$; found 384.0197

$N$-phenyl-N'-(pyridin-2-yl)morpholine-4-carboximidamide (3i) : According to the general procedure, the reaction was performed by using 1-phenyl-3-(pyridin-2-yl)thiourea (1i, $115 \mathrm{mg}, 0.5$ mmol), morpholine (174 mg, $2.0 \mathrm{mmol}$ ), $\mathrm{Ru}(\mathrm{bpy})_{3} \mathrm{Cl}_{2} \cdot 6 \mathrm{H}_{2} \mathrm{O}$ (4 mg, $\left.0.005 \mathrm{mmol}\right)$, and $\mathrm{K}_{2} \mathrm{CO}_{3}(138$ $\mathrm{mg}, 1.0 \mathrm{mmol})$ in $5 \mathrm{~mL}$ of $\mathrm{EtOH}: \mathrm{H}_{2} \mathrm{O}(9: 1)$ for $24 \mathrm{~h}$ to afford $\mathbf{3 i}(86 \mathrm{mg}, 0.31 \mathrm{mmol}, 61 \%)$ as a yellow solid ${ }^{1} \mathrm{H}$ NMR (400 MHz, $\left.\mathrm{CDCl}_{3}\right) \delta 7.53(\mathrm{~s}, J=8.4 \mathrm{~Hz}, 2 \mathrm{H}), 7.29(\mathrm{~d}, J=7.1 \mathrm{~Hz}, 2 \mathrm{H}), 7.02$ 
$-6.97(\mathrm{~m}, 3 \mathrm{H}), 6.71(\mathrm{~d}, J=7.9 \mathrm{~Hz}, 2 \mathrm{H}), 3.69(\mathrm{~m}, 4 \mathrm{H}), 3.34(\mathrm{~m}, 4 \mathrm{H}) . ;{ }^{13} \mathrm{C} \mathrm{NMR}\left(100 \mathrm{MHz}, \mathrm{CDCl}_{3}\right)$

$\delta$ 150.9, 138.3, 129.5, 122.9, 119.7, 66.3, 47.1.; HRMS (ESI/Q-TOF) m/z:[M+H] ${ }^{+}$calcd for $\mathrm{C}_{16} \mathrm{H}_{19} \mathrm{~N}_{4} \mathrm{O} 283.1599$; found 283.1625

$N^{\prime}$-benzyl-N-phenylmorpholine-4-carboximidamide $(3 j)^{8}$ : According to the general procedure, the reaction was performed by using 1-benzyl-3-phenylthiourea $(\mathbf{1 j}, 121 \mathrm{mg}, 0.5 \mathrm{mmol})$, morpholine (174 mg, $2.0 \mathrm{mmol}), \mathrm{Ru}(\mathrm{bpy})_{3} \mathrm{Cl}_{2} \cdot 6 \mathrm{H}_{2} \mathrm{O}(20 \mathrm{mg}, 0.025 \mathrm{mmol})$, and $\mathrm{K}_{2} \mathrm{CO}_{3}(138 \mathrm{mg}, 1.0 \mathrm{mmol})$ in $5 \mathrm{~mL}$ of EtOH: $\mathrm{H}_{2} \mathrm{O}(9: 1)$ for $24 \mathrm{~h}$ to afford $\mathbf{3 j}$ ( $32 \mathrm{mg}, 0.11 \mathrm{mmol}, 22 \%$ ) as a yellow solid ${ }^{1} \mathrm{H}$ NMR (400 MHz, $\mathrm{CDCl}_{3} \delta 7.35-7.25(\mathrm{~m}, 7 \mathrm{H}), 6.97(\mathrm{t}, J=7.4 \mathrm{~Hz}, 1 \mathrm{H}), 6.76(\mathrm{~d}, J=7.4 \mathrm{~Hz}, 2 \mathrm{H}), 4.31$ (s, 2H), $3.76-3.74(\mathrm{~m}, 4 \mathrm{H}), 3.32-3.29(\mathrm{~m}, 4 \mathrm{H}) . ;{ }^{13} \mathrm{C} \mathrm{NMR}\left(100 \mathrm{MHz}, \mathrm{CDCl}_{3}\right) \delta$ 155.9, 138.6, 129.3, 128.7, 127.6, 127.5, 122.5, 122.1, 66.6, 49.1, 48.3.; HRMS (ESI/Q-TOF) m/z:[M+H] ${ }^{+}$calcd for $\mathrm{C}_{18} \mathrm{H}_{22} \mathrm{~N}_{3} \mathrm{O} 296.1763$; found 296.1766

\section{General procedure for one-pot synthesis of guanidine from the isothiocyanate}

A Pyrex glass tube equipped with a magnetic stirring bar was charged with $\mathrm{K}_{2} \mathrm{CO}_{3}(1.0 \mathrm{mmol})$ and $\mathrm{Ru}(\mathrm{bpy})_{3} \mathrm{Cl}_{2} 6 \mathrm{H}_{2} \mathrm{O}(1 \mathrm{~mol} \%)$. Isothiocyanatobenzene $(4,0.5 \mathrm{mmol}), p$-toluidine $(0.75 \mathrm{mmol})$ amine (2.0 mmol), and $5 \mathrm{~mL}$ ethanol: $\mathrm{H}_{2} \mathrm{O}$ (9:1) were added and placed in the 19W LED light reactor. The reaction mixture was stirred at room temperature for $24 \mathrm{~h}$ under $19 \mathrm{~W}$ white LED irradiation. The mixture was filtered, and the residue was purified by chromatography (hexane/ethyl acetate).

$N^{\prime}$-phenyl- $N$-(p-tolyl)morpholine-4-carboximidamide (3c): According to the general procedure, the reaction was performed by using isothiocyanatobenzene $(4,68 \mathrm{mg}, 0.5 \mathrm{mmol}), p$-toluidine ( 80 $\mathrm{mg}, 0.75 \mathrm{mmol})$, morpholine (174 mg, $2.0 \mathrm{mmol}), \mathrm{Ru}(\mathrm{bpy}){ }_{3} \mathrm{Cl}_{2} \cdot 6 \mathrm{H}_{2} \mathrm{O}(4 \mathrm{mg}, 0.005 \mathrm{mmol})$, and 
$\mathrm{K}_{2} \mathrm{CO}_{3}(138 \mathrm{mg}, 1.0 \mathrm{mmol})$ in $5 \mathrm{~mL}$ of $\mathrm{EtOH}: \mathrm{H}_{2} \mathrm{O}(9: 1)$ for $24 \mathrm{~h}$ to afford $\mathbf{3 c}(91 \mathrm{mg}, 0.31 \mathrm{mmol}$, $62 \%)$ as a yellow solid ${ }^{1} \mathrm{H} \mathrm{NMR}\left(500 \mathrm{MHz}, \mathrm{CDCl}_{3}\right) \delta 7.50(\mathrm{~d}, J=8.6 \mathrm{~Hz}, 2 \mathrm{H}), 7.24(\mathrm{t}, J=7.9 \mathrm{~Hz}$, 2H), $6.98(\mathrm{t}, J=7.4 \mathrm{~Hz}, 1 \mathrm{H}), 6.92(\mathrm{~d}, J=6.6 \mathrm{~Hz}, 2 \mathrm{H}), 6.66(\mathrm{~d}, J=7.5 \mathrm{~Hz}, 2 \mathrm{H}), 3.66-3.64(\mathrm{~m}$, 4H), $3.31-3.30(\mathrm{~m}, 4 \mathrm{H}), 1.24(\mathrm{~s}, 3 \mathrm{H}) . ; 13^{\mathrm{C}} \mathrm{NMR}\left(125 \mathrm{MHz}, \mathrm{CDCl}_{3}\right) \delta 150.9,138.3,129.6,122.8$, $66.4,47.1,29.8,29.5$

\section{Intermediate investigation experiment (Equation 1)}

A Pyrex glass tube equipped with a magnetic stirring bar and charged with 1,3-diphenylthiourea (1a, $114 \mathrm{mg}, 0.5 \mathrm{mmol}), \mathrm{Ru}(\mathrm{bpy})_{3} \mathrm{Cl}_{2} \cdot 6 \mathrm{H}_{2} \mathrm{O}(4 \mathrm{mg}, 0.005 \mathrm{mmol}), \mathrm{K}_{2} \mathrm{CO}_{3}(138 \mathrm{mg}, 1.0 \mathrm{mmol})$ in 5 $\mathrm{mL}$ of EtOH: $\mathrm{H}_{2} \mathrm{O}(9: 1)$. The reaction was then placed in the 19W white LED light reactor and stirred at room temperature for $24 \mathrm{~h}$. Small amount of reaction mixture was sampled and characterized by mass spectrometry. MS: m/z: 213.1 (Figure S34)

\section{By-product investigation experiment (Equation 2)}

A Pyrex glass tube equipped with a magnetic stirring bar was charged with DBU (1.0 mmol) and $\mathrm{Ru}(\mathrm{bpy})_{3} \mathrm{Cl}_{2} \cdot 6 \mathrm{H}_{2} \mathrm{O}$ (1 mol\%). 1,3-diphenylthiourea (1a, $\left.0.5 \mathrm{mmol}\right)$, morpholine (2.0 mmol), and 5 $\mathrm{mL}$ of $\mathrm{MeCN}$ were added and placed in the $19 \mathrm{~W}$ white LED light reactor. The reaction mixture was stirred at room temperature for $24 \mathrm{~h}$ under $19 \mathrm{~W}$ white LED irradiation. After 1a were completely consumed, a lead acetate $(0.5 \mathrm{mmol})$ was added. The mixture was filtered, washed, and dried under vacuum. The precipitate was collected for SEM/EDX characterization. The morphological structure of particles was observed using a scanning electron microscope (SEM, JEOL JSM-6510A, Figure S62). The elemental composition analysis of the material was investigated using a built-in energy dispersive X-ray spectrometer (EDS, Table S1). 


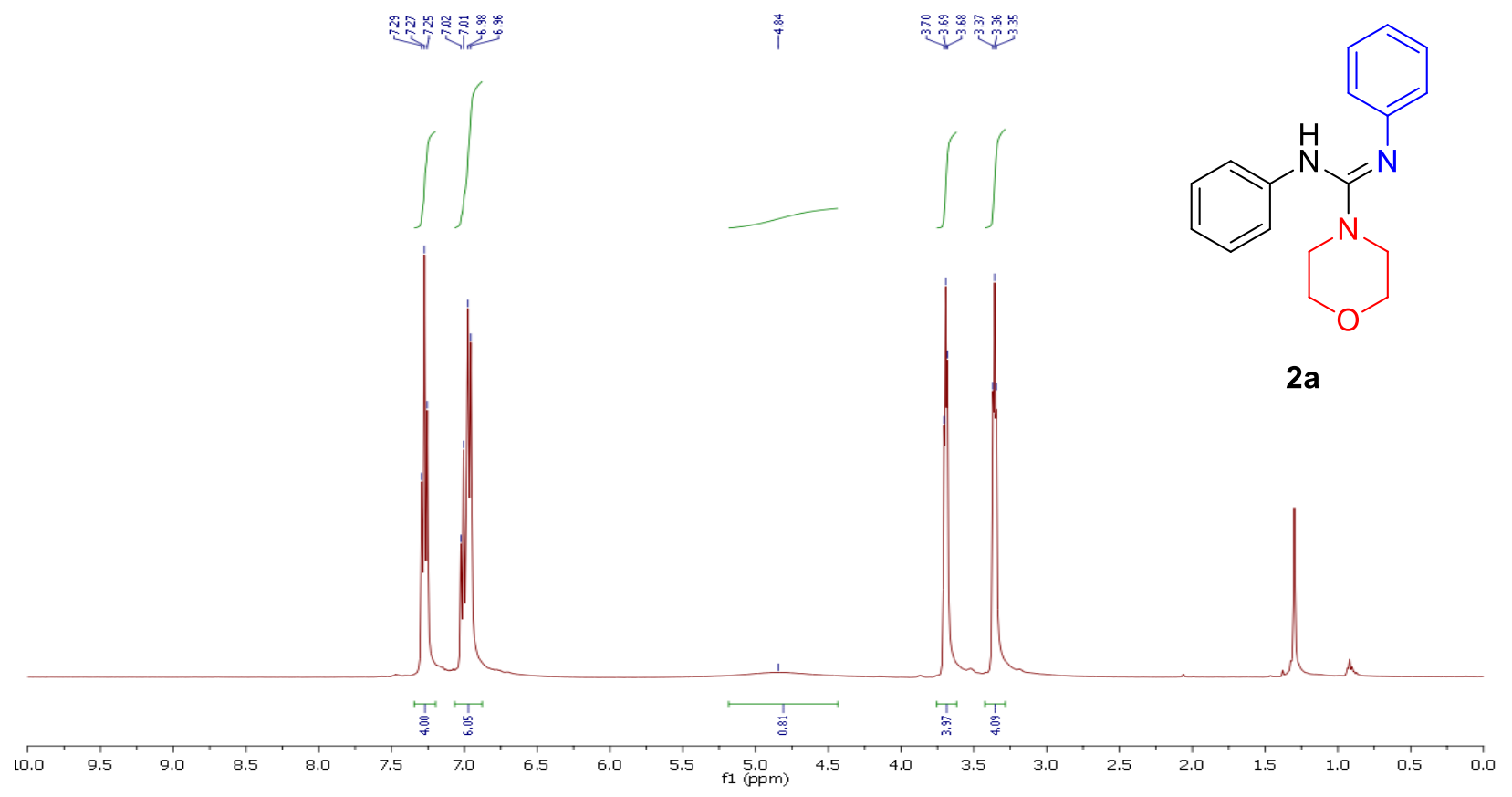

Figure S3. ${ }^{1} \mathrm{H}$ NMR spectrum of 2 a (400 MHz, CDCl 3$)$
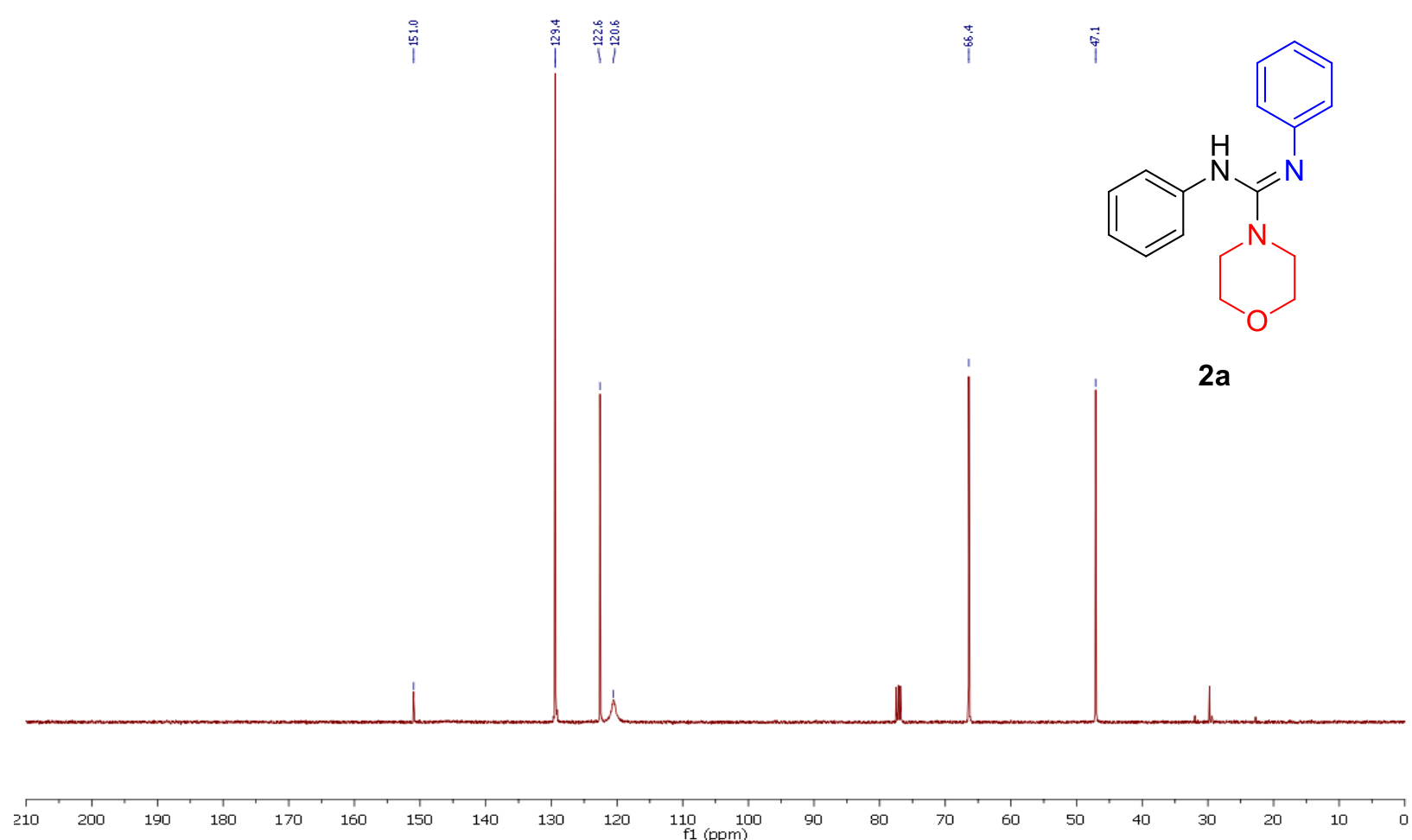

Figure S4. ${ }^{13} \mathrm{C}$ NMR spectrum of $2 \mathbf{a}\left(\mathbf{1 0 0} \mathbf{~ M H z}, \mathbf{C D C l}_{3}\right)$ 


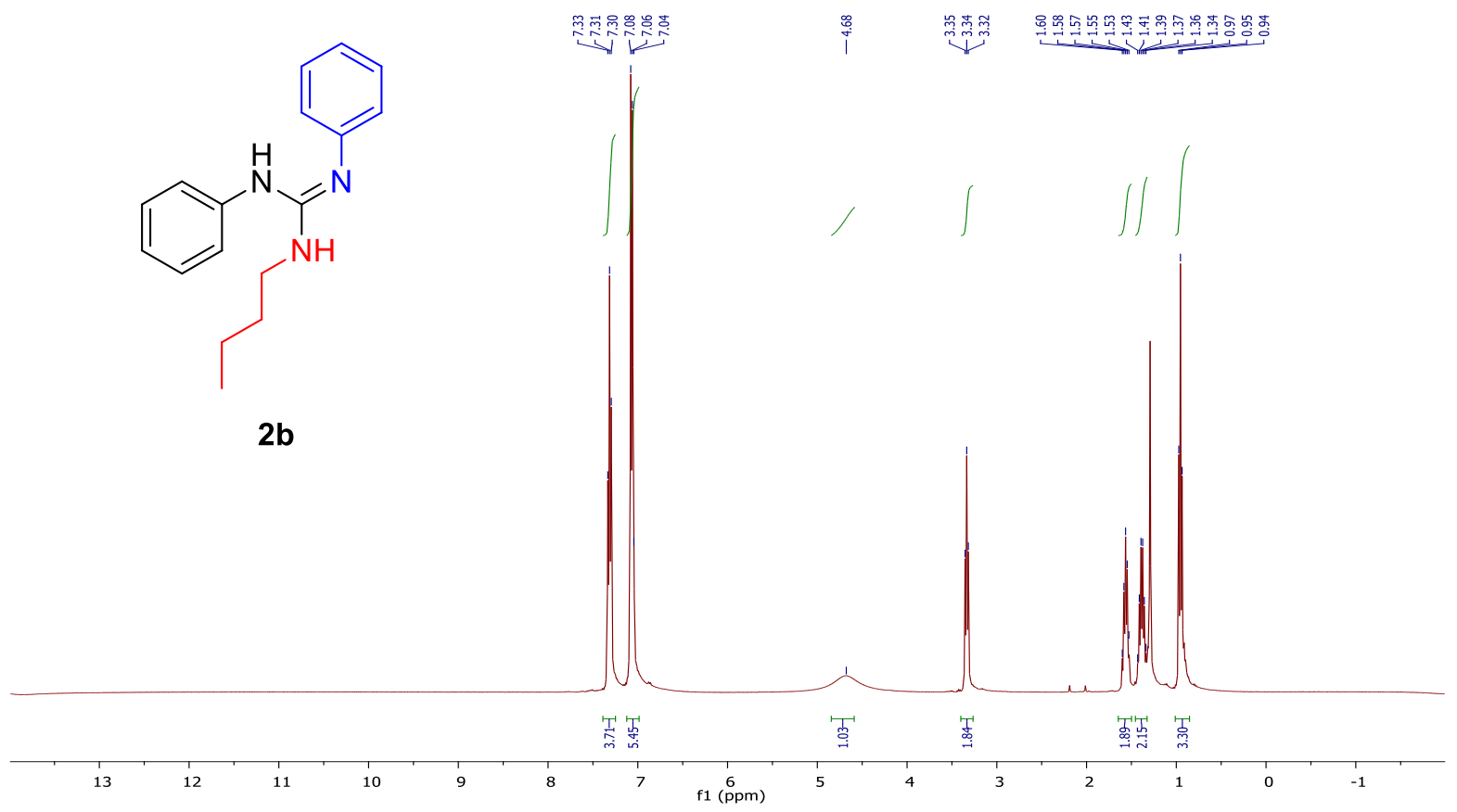

Figure S5. ${ }^{1} \mathrm{H}$ NMR spectrum of $2 \mathrm{~b}\left(400 \mathrm{MHz}, \mathrm{CDCl}_{3}\right)$

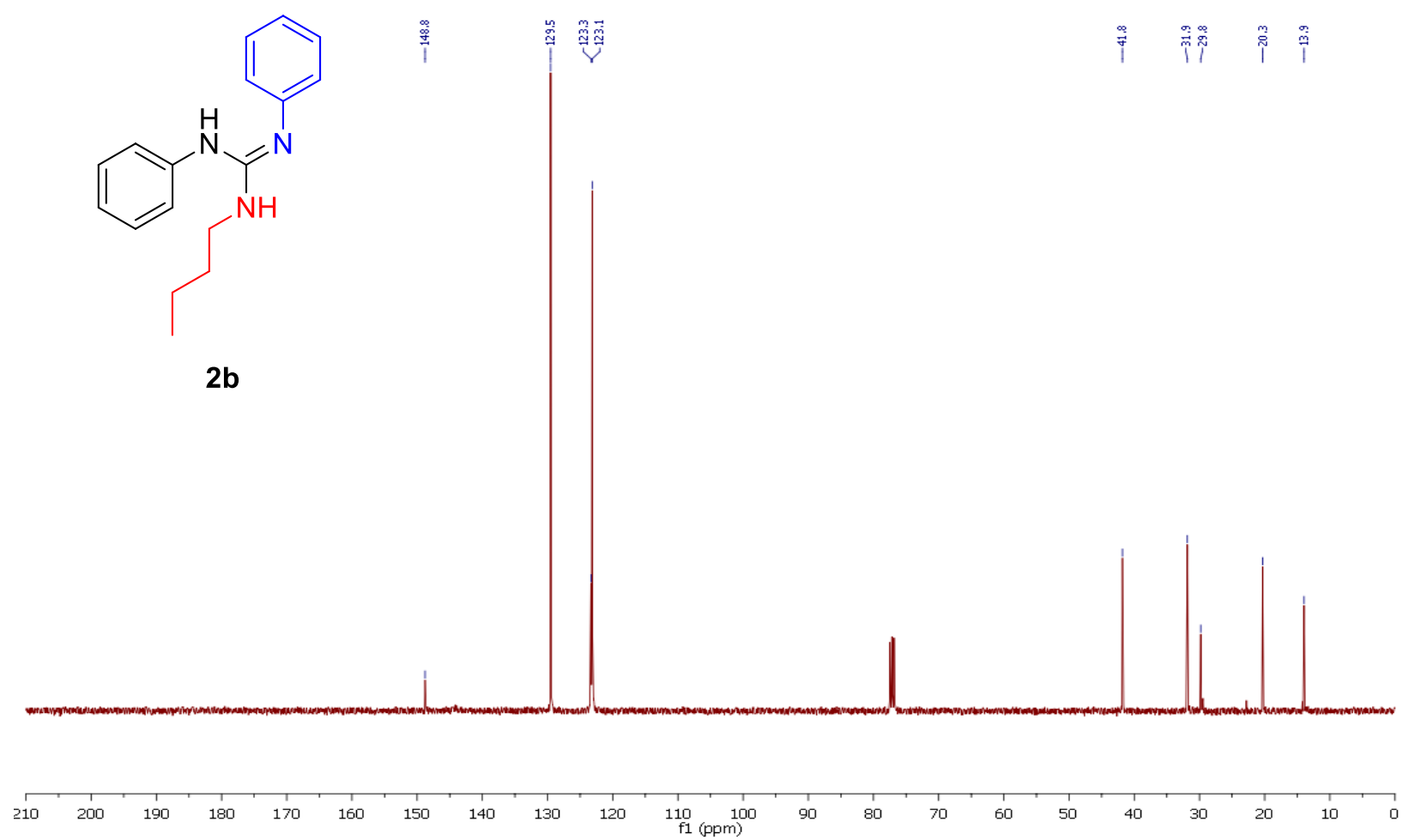

Figure S6. ${ }^{13} \mathrm{C}$ NMR spectrum of $2 \mathrm{~b}\left(100 \mathrm{MHz}, \mathrm{CDCl}_{3}\right)$ 


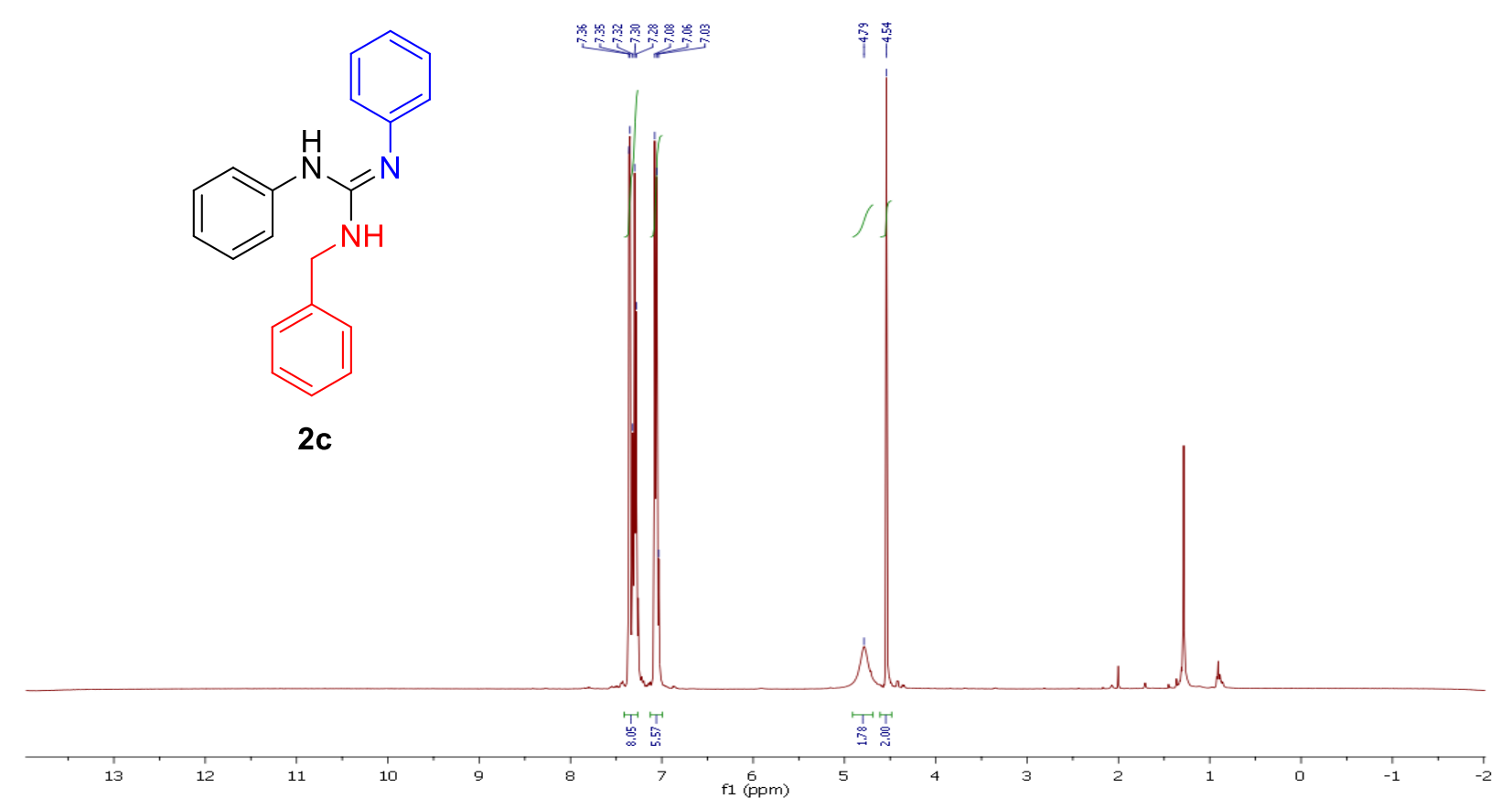

Figure S7. ${ }^{1} \mathrm{H}$ NMR spectrum of $2 \mathrm{c}\left(400 \mathrm{MHz}, \mathrm{CDCl}_{3}\right)$

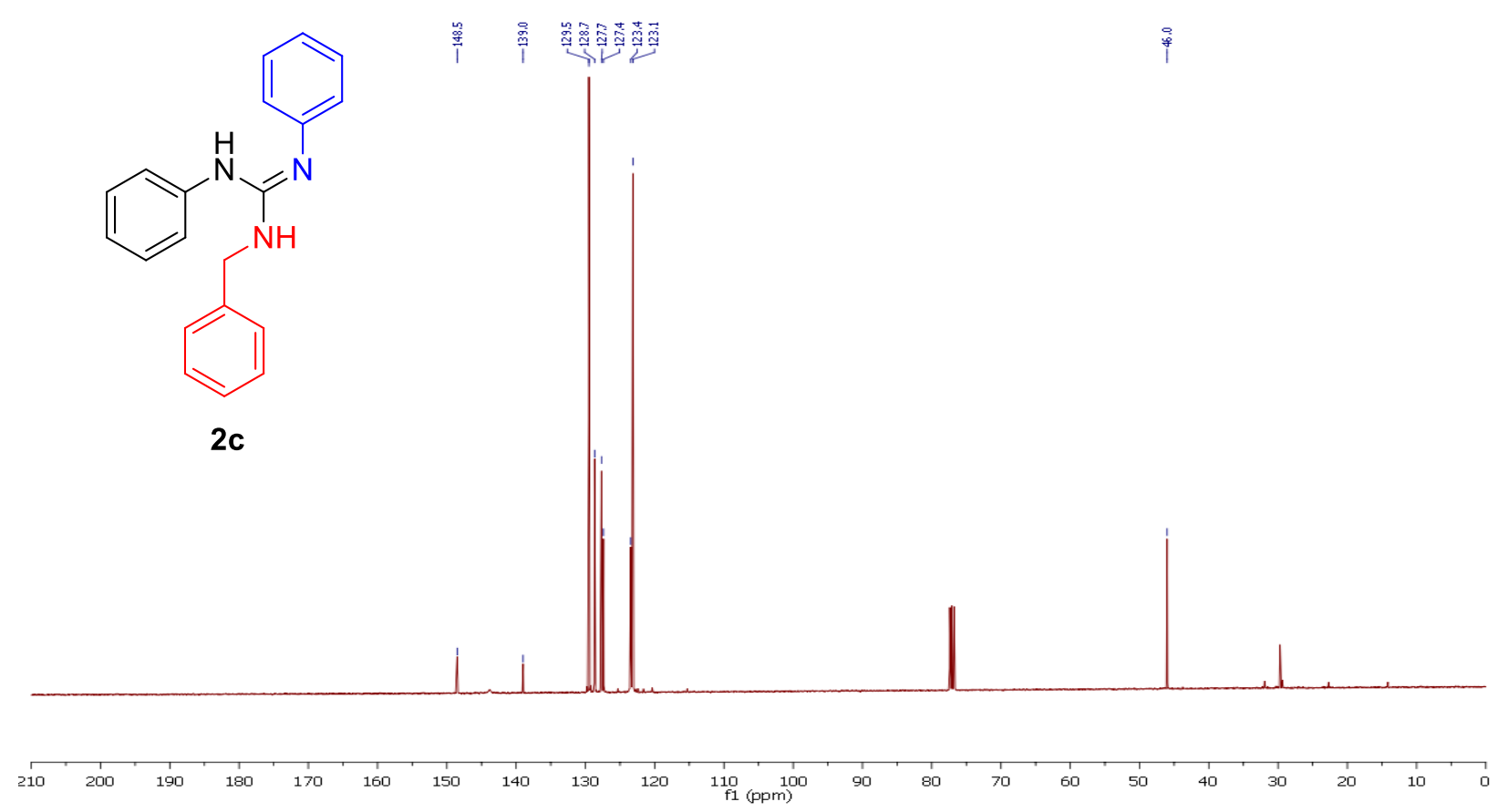

Figure S8. ${ }^{13} \mathrm{C}$ NMR spectrum of 2 c (100 $\left.\mathbf{~ M H z , ~} \mathbf{C D C l}_{3}\right)$ 


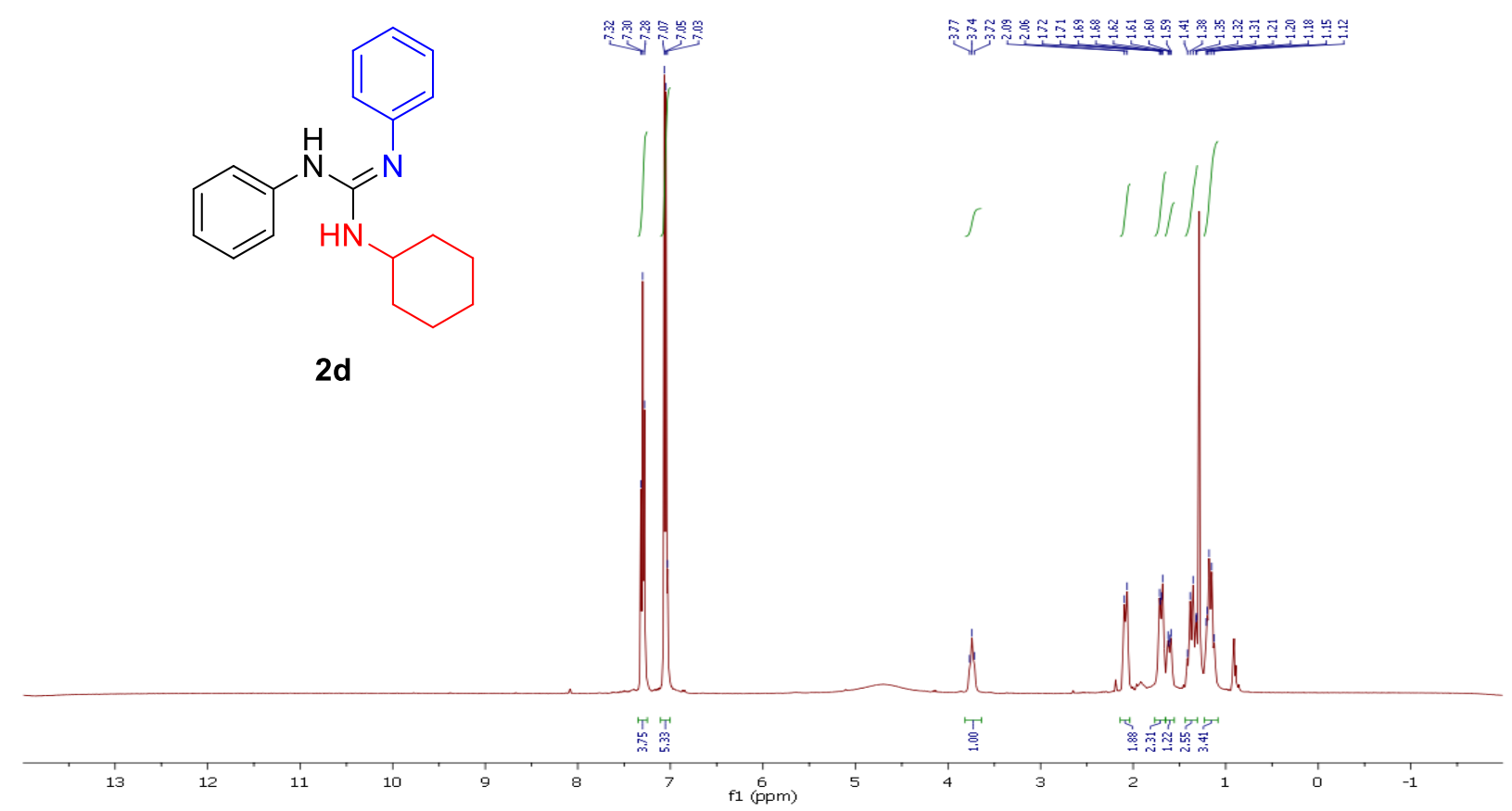

Figure S9. ${ }^{1} \mathrm{H}$ NMR spectrum of $2 \mathrm{~d}\left(400 \mathrm{MHz}, \mathrm{CDCl}_{3}\right)$

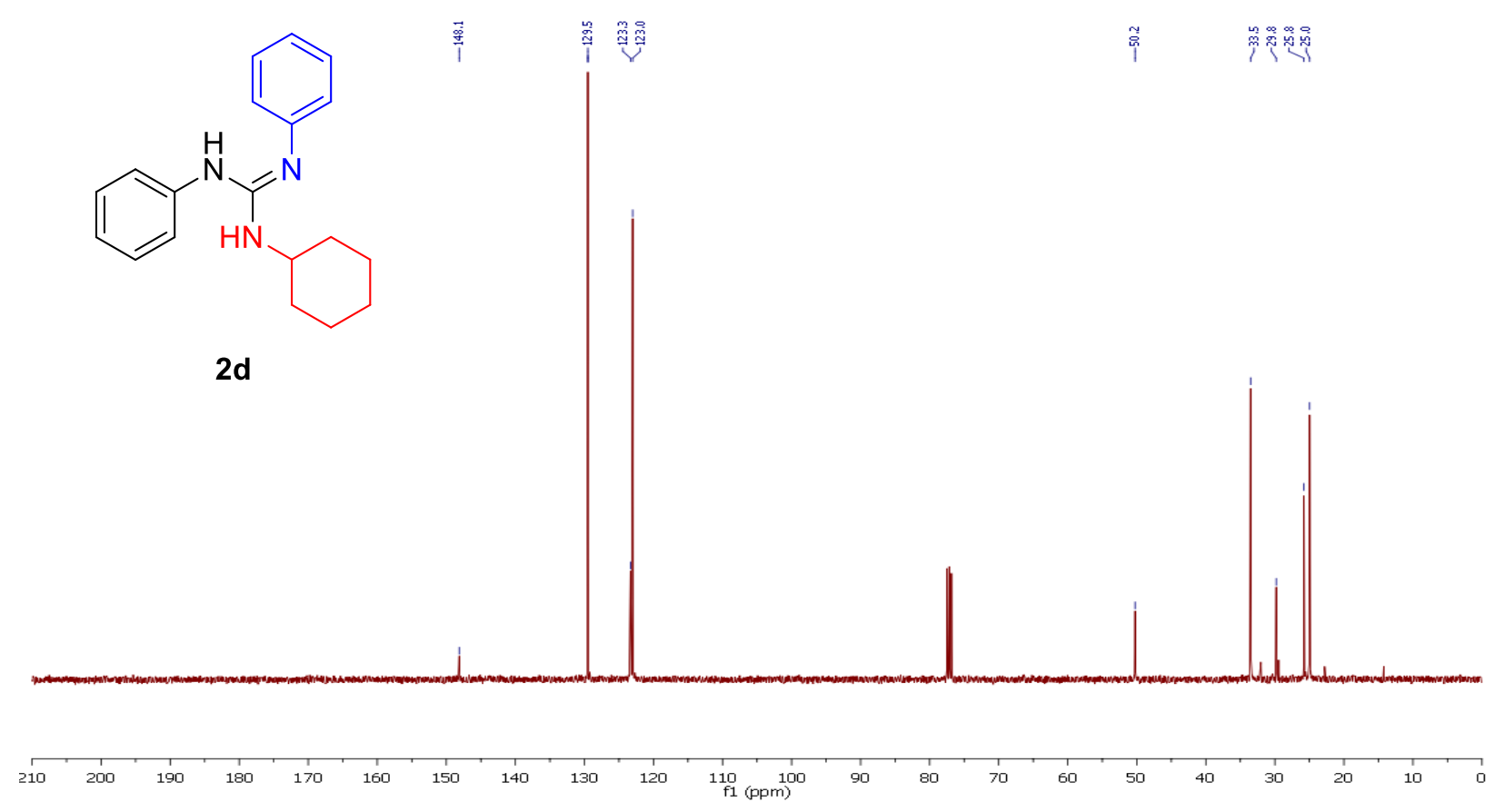

Figure S10. ${ }^{13} \mathrm{C}$ NMR spectrum of $2 \mathrm{~d}\left(\mathbf{1 0 0} \mathrm{MHz}, \mathrm{CDCl}_{3}\right)$ 

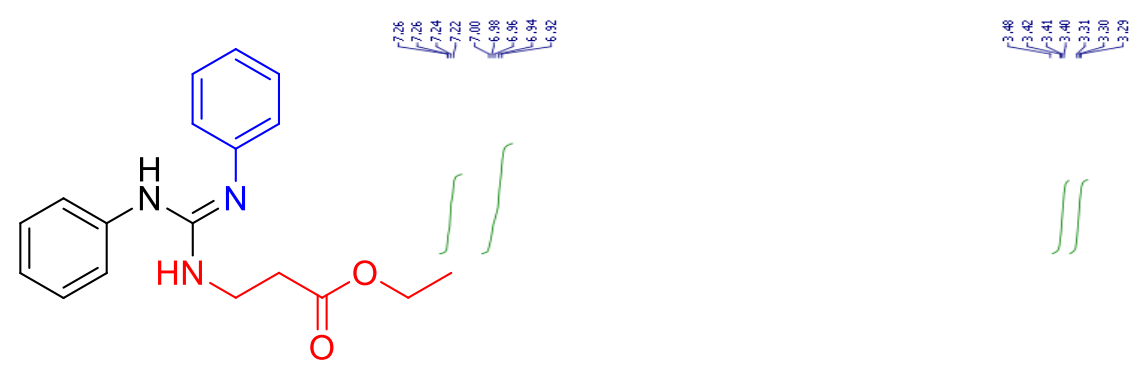

$2 e$

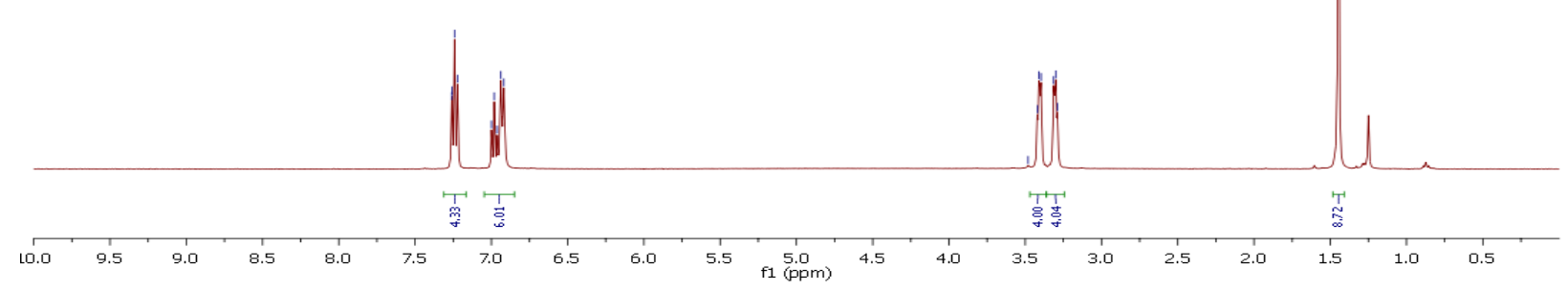

Figure S11. ${ }^{1} \mathrm{H}$ NMR spectrum of $2 \mathrm{e}\left(400 \mathrm{MHz}, \mathrm{CDCl}_{3}\right)$
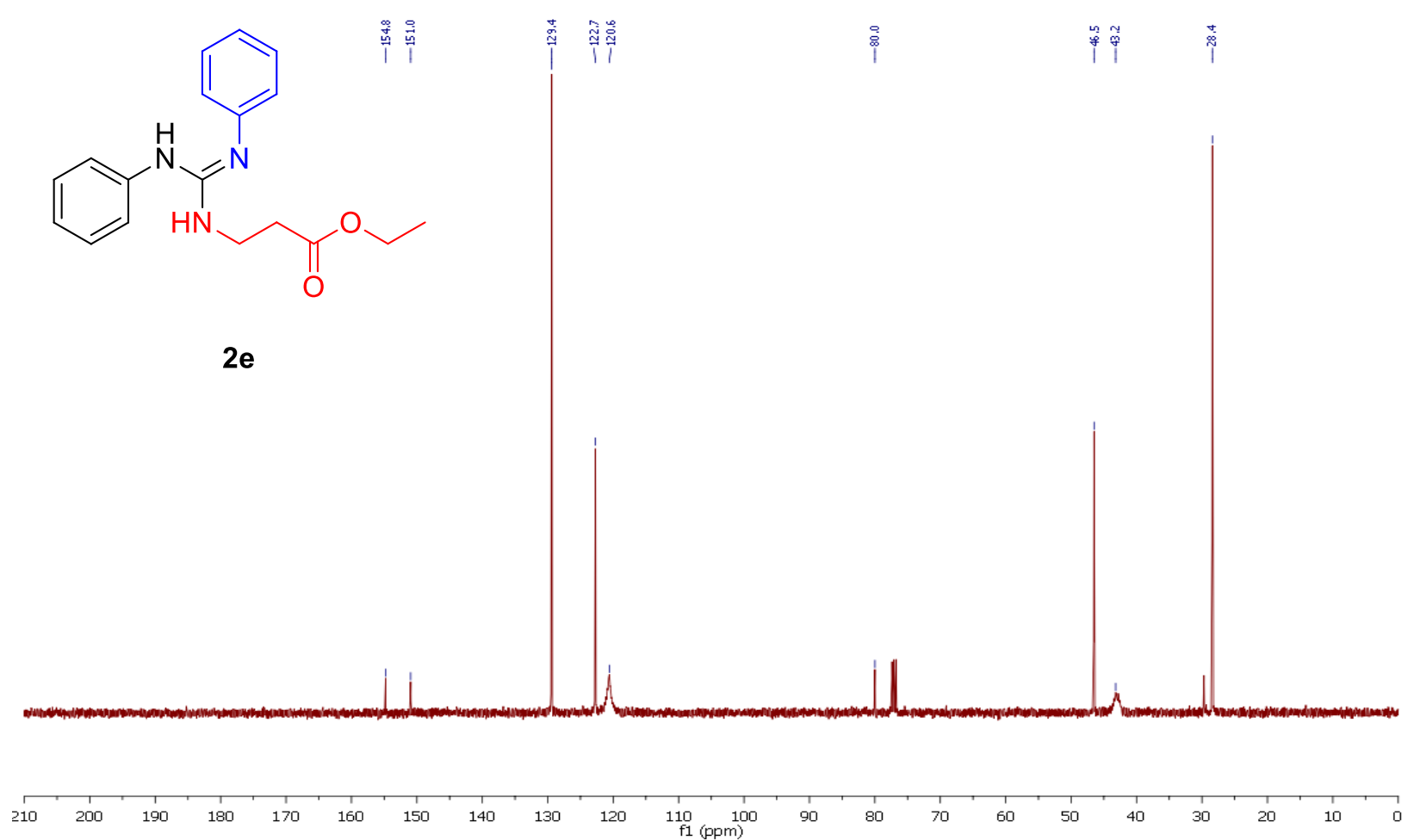

Figure S12. ${ }^{13} \mathrm{C}$ NMR spectrum of $2 \mathrm{e}\left(100 \mathrm{MHz}, \mathrm{CDCl}_{3}\right)$ 


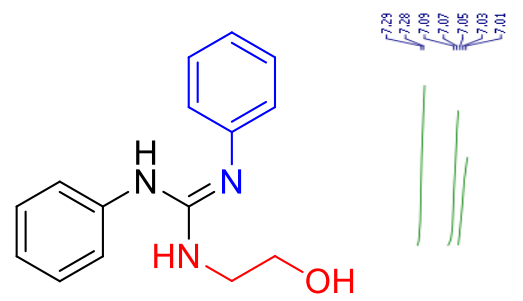

2f

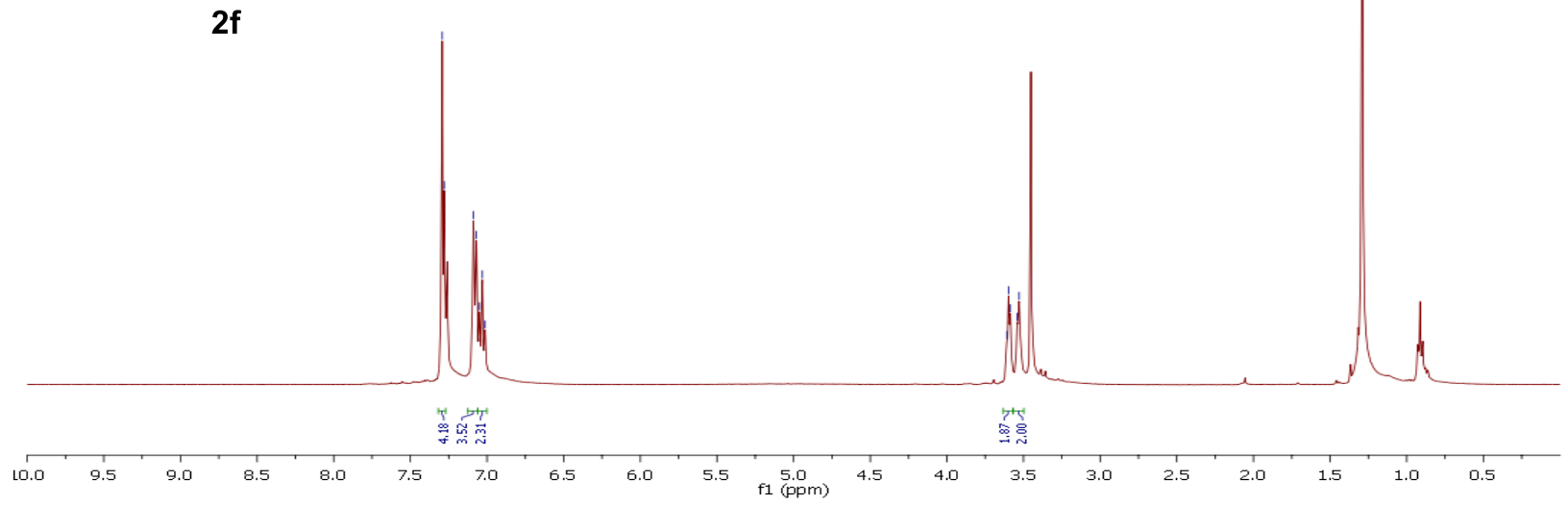

Figure S13. ${ }^{1} \mathrm{H}$ NMR spectrum of $2 \mathrm{f}\left(400 \mathrm{MHz}, \mathrm{CDCl}_{3}\right)$

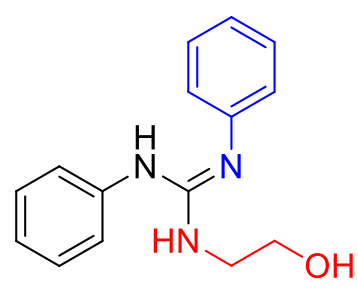

$2 f$

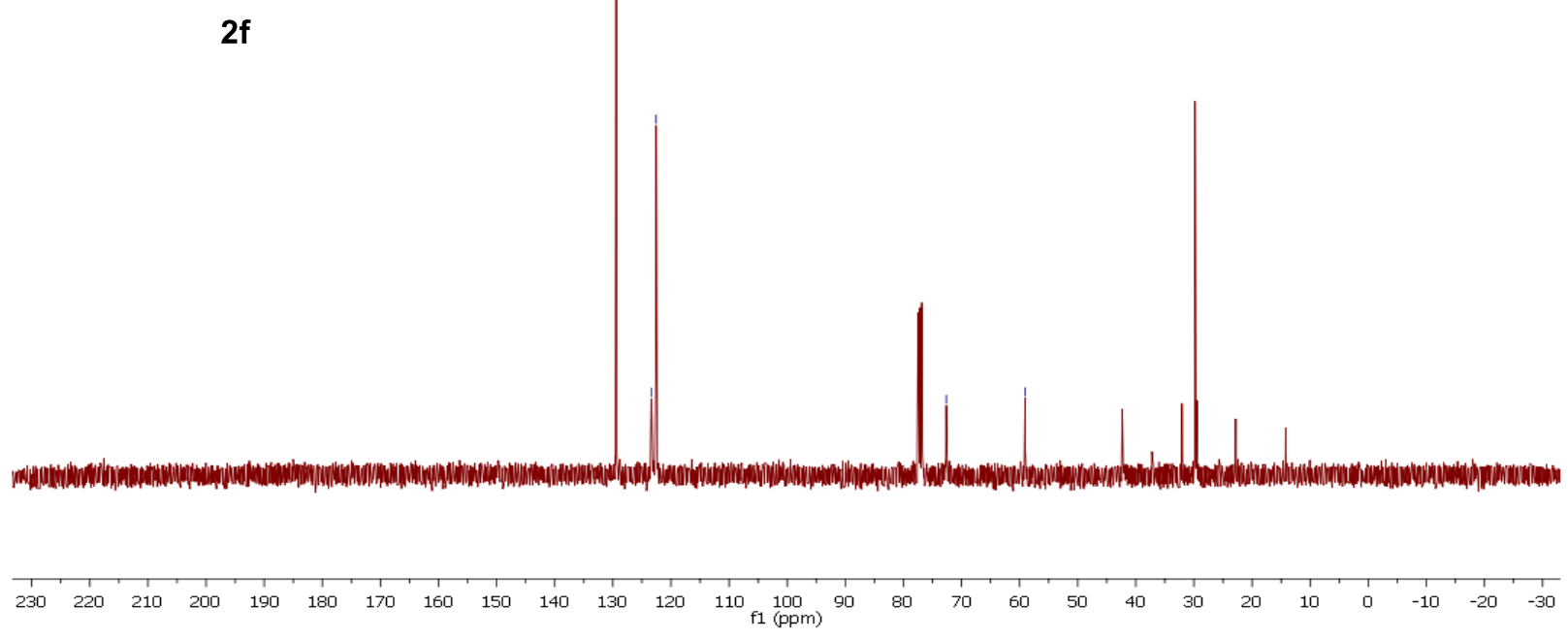

Figure S14. ${ }^{13} \mathrm{C}$ NMR spectrum of $2 \mathrm{f}(100 \mathrm{MHz}, \mathrm{CDCl})$ 


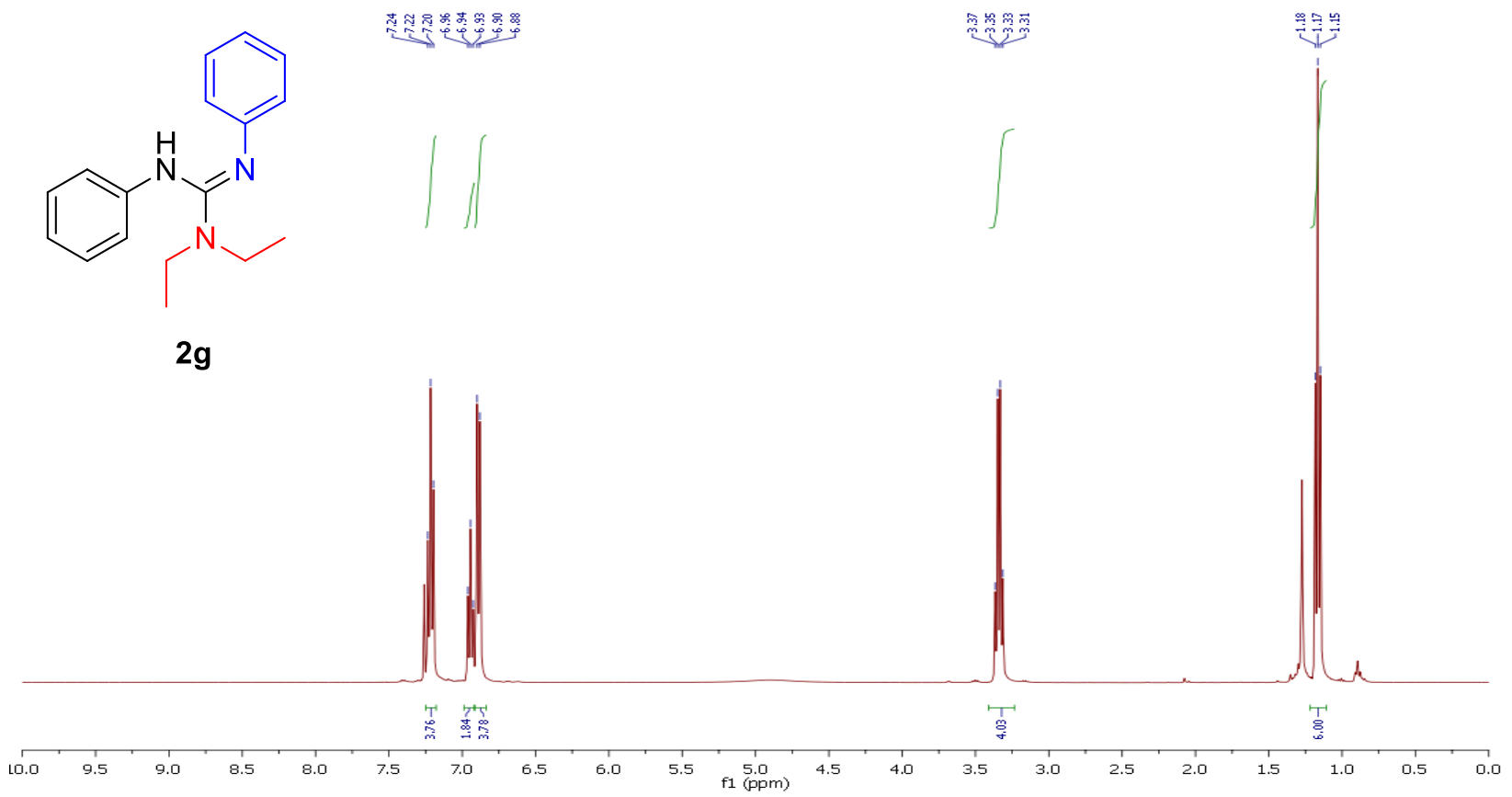

Figure S15. ${ }^{1} \mathrm{H}$ NMR spectrum of $2 \mathrm{~g}\left(400 \mathrm{MHz}, \mathrm{CDCl}_{3}\right)$

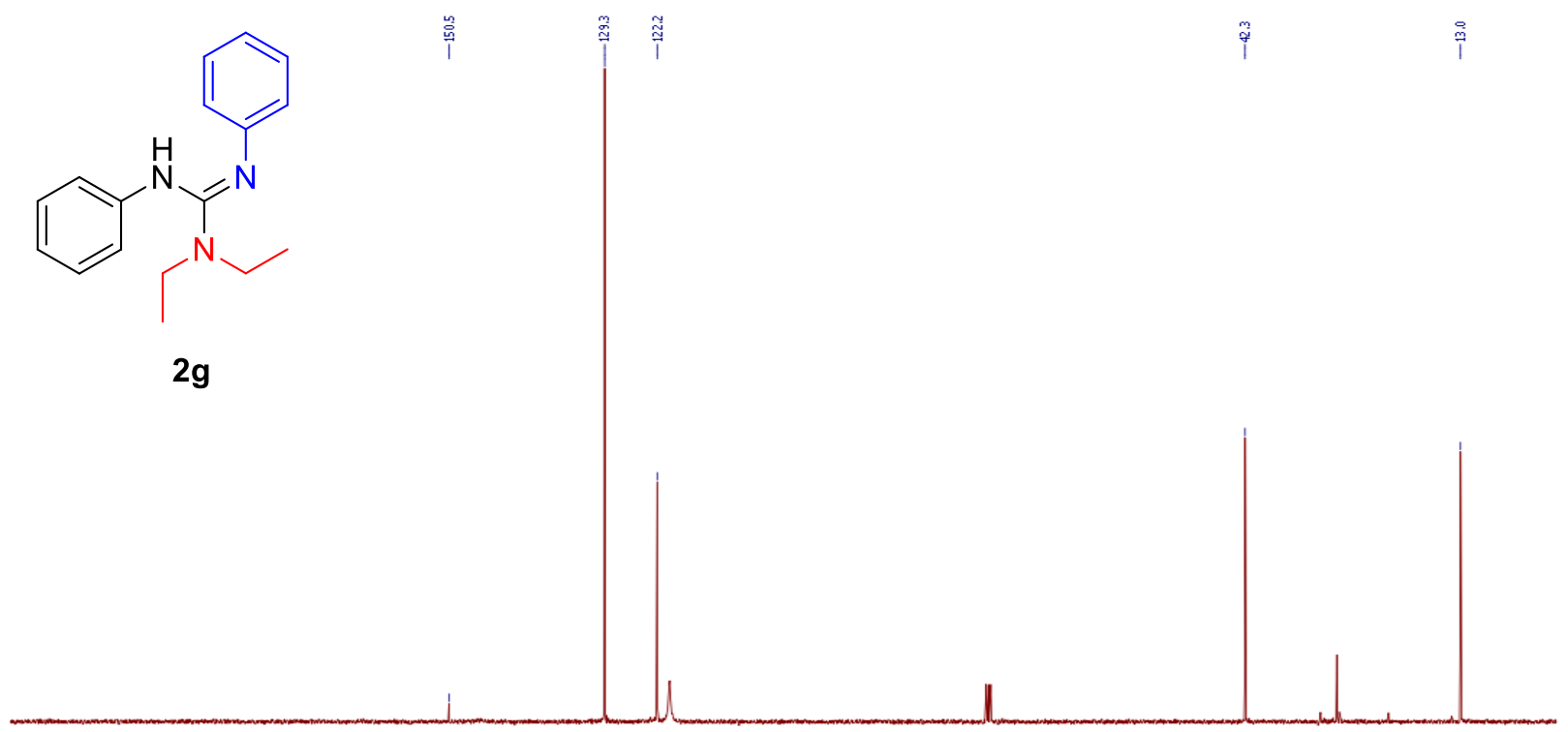

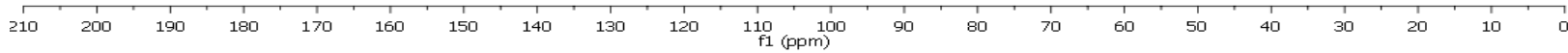

Figure S16. ${ }^{13} \mathrm{C}$ NMR spectrum of $2 \mathrm{~g}\left(100 \mathrm{MHz}, \mathrm{CDCl}_{3}\right)$ 


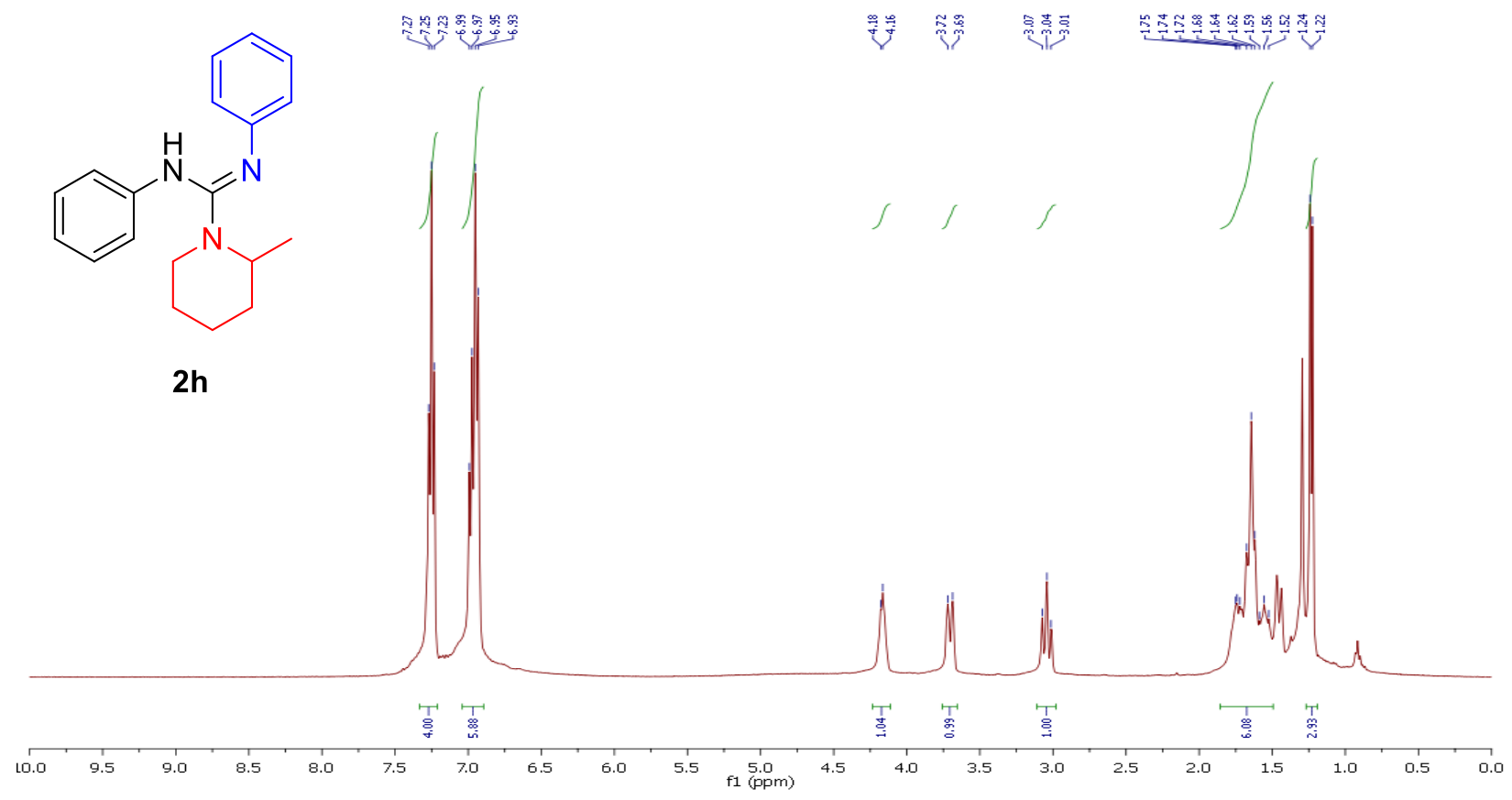

Figure S17. ${ }^{1} \mathrm{H}$ NMR spectrum of $2 \mathrm{~h}\left(400 \mathrm{MHz}, \mathrm{CDCl}_{3}\right)$

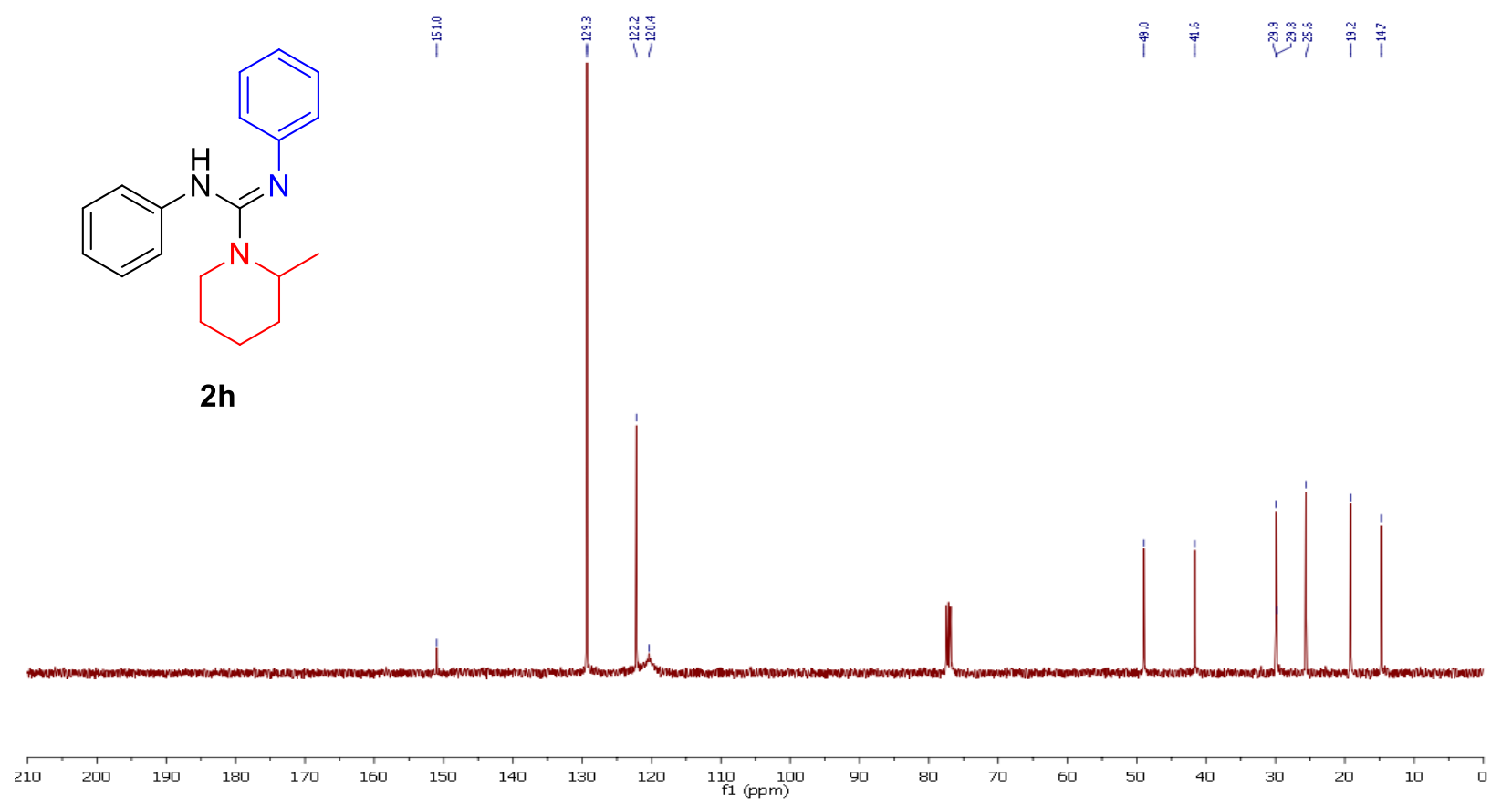

Figure S18. ${ }^{13} \mathrm{C}$ NMR spectrum of $2 \mathrm{~h}\left(100 \mathrm{MHz} \mathrm{CDCl}_{3}\right)$ 


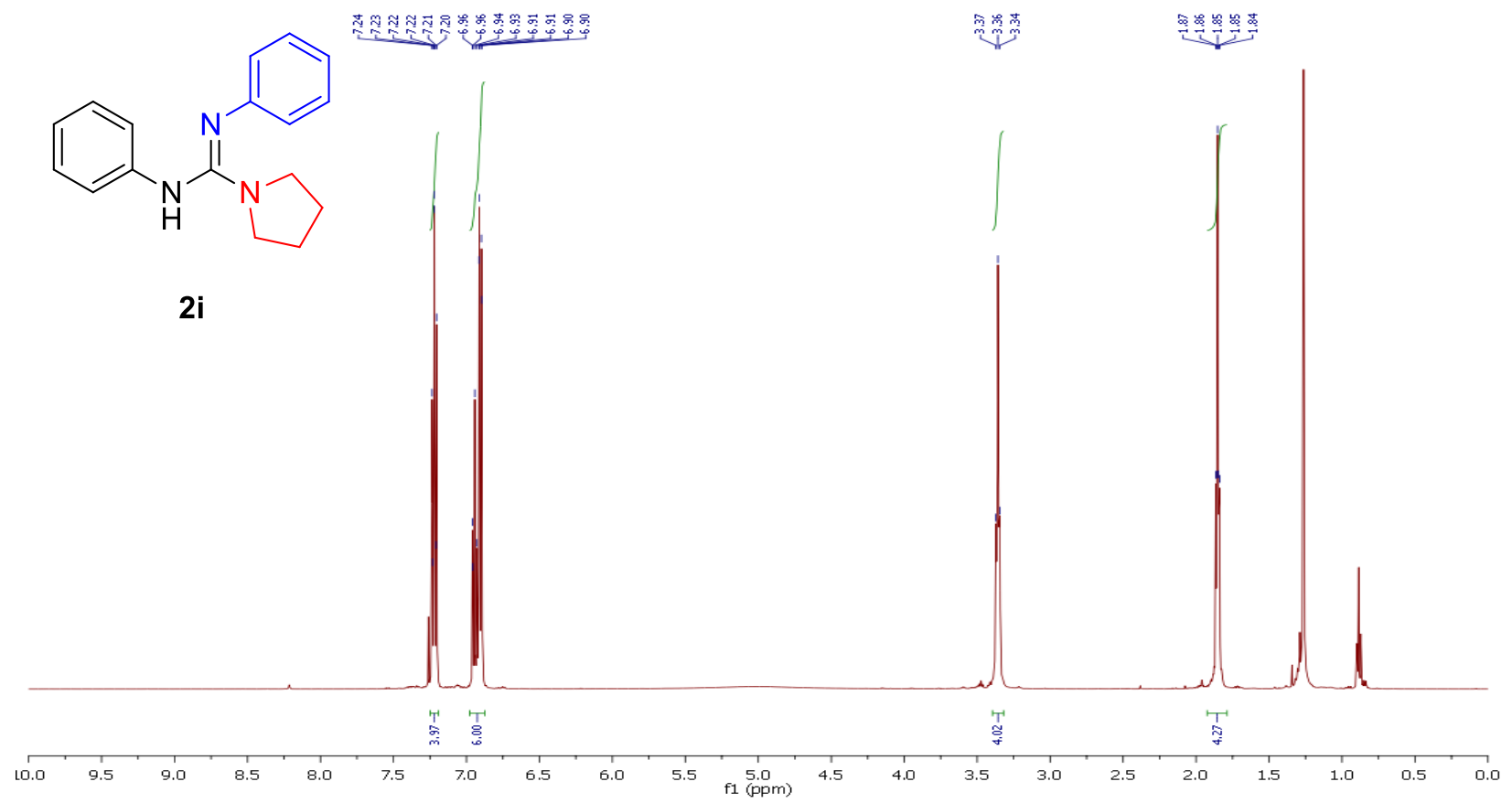

Figure S19. ${ }^{1} \mathrm{H}$ NMR spectrum of $2 \mathbf{i}\left(500 \mathrm{MHz}, \mathbf{C D C l}_{3}\right)$
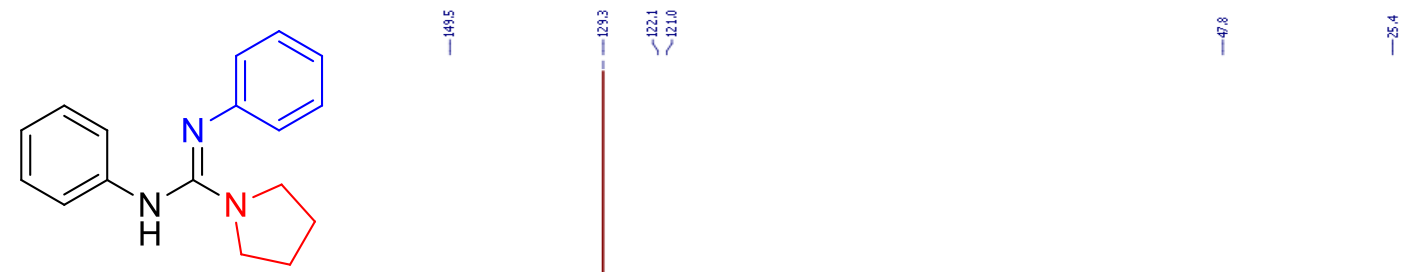

2i

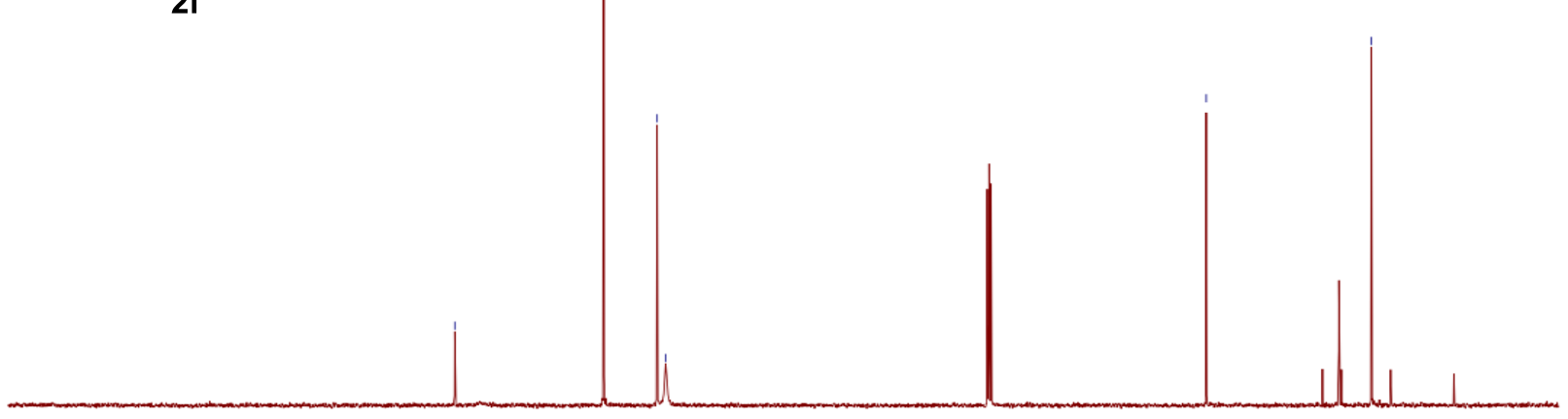

Figure S20. ${ }^{13} \mathrm{C}$ NMR spectrum of $2 \mathbf{i}\left(\mathbf{1 2 5} \mathbf{M H z}, \mathbf{C D C l}_{3}\right)$ 


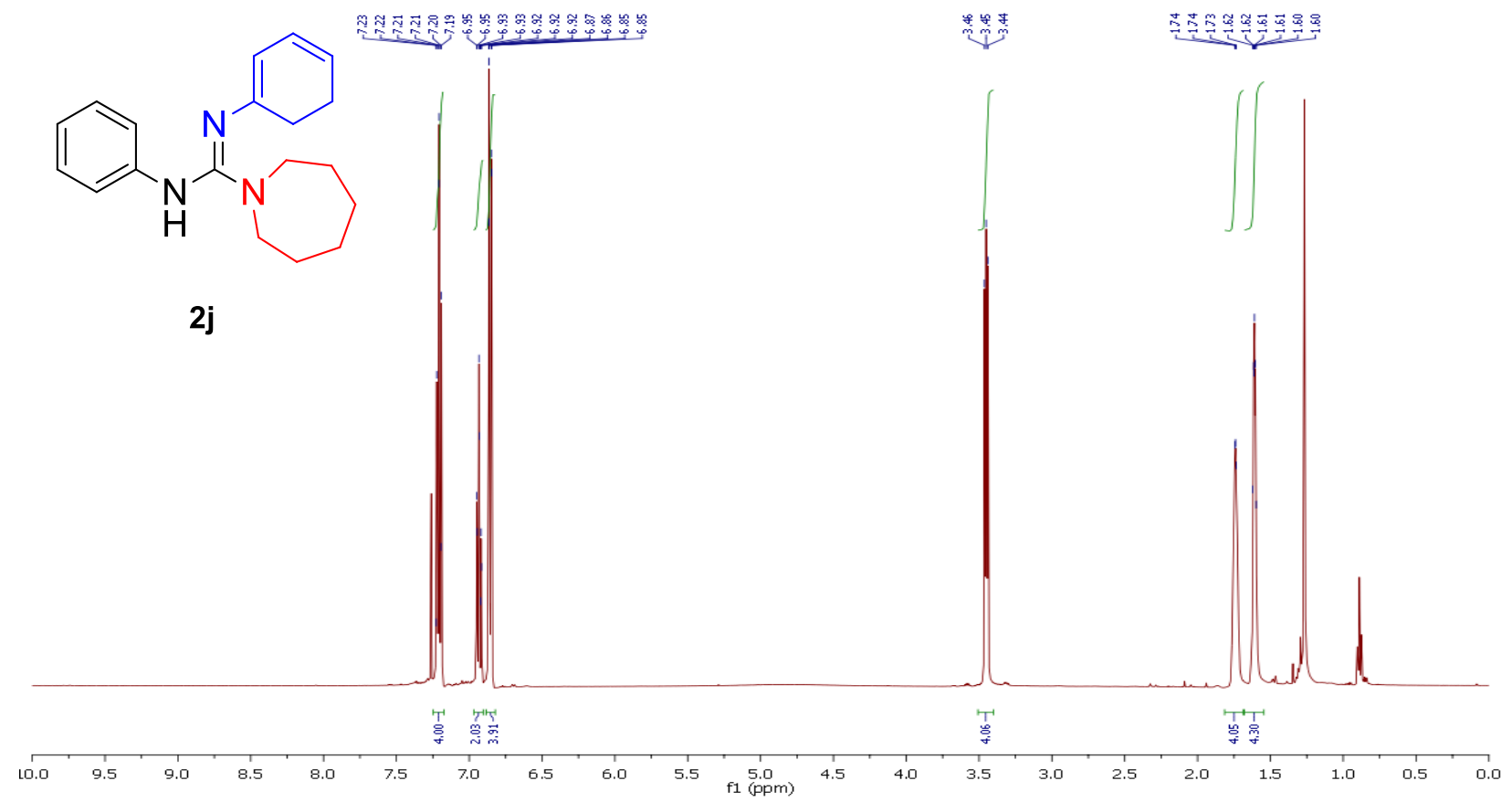

Figure S21. ${ }^{1} \mathrm{H}$ NMR spectrum of $2 \mathbf{j}\left(500 \mathrm{MHz}, \mathrm{CDCl}_{3}\right)$
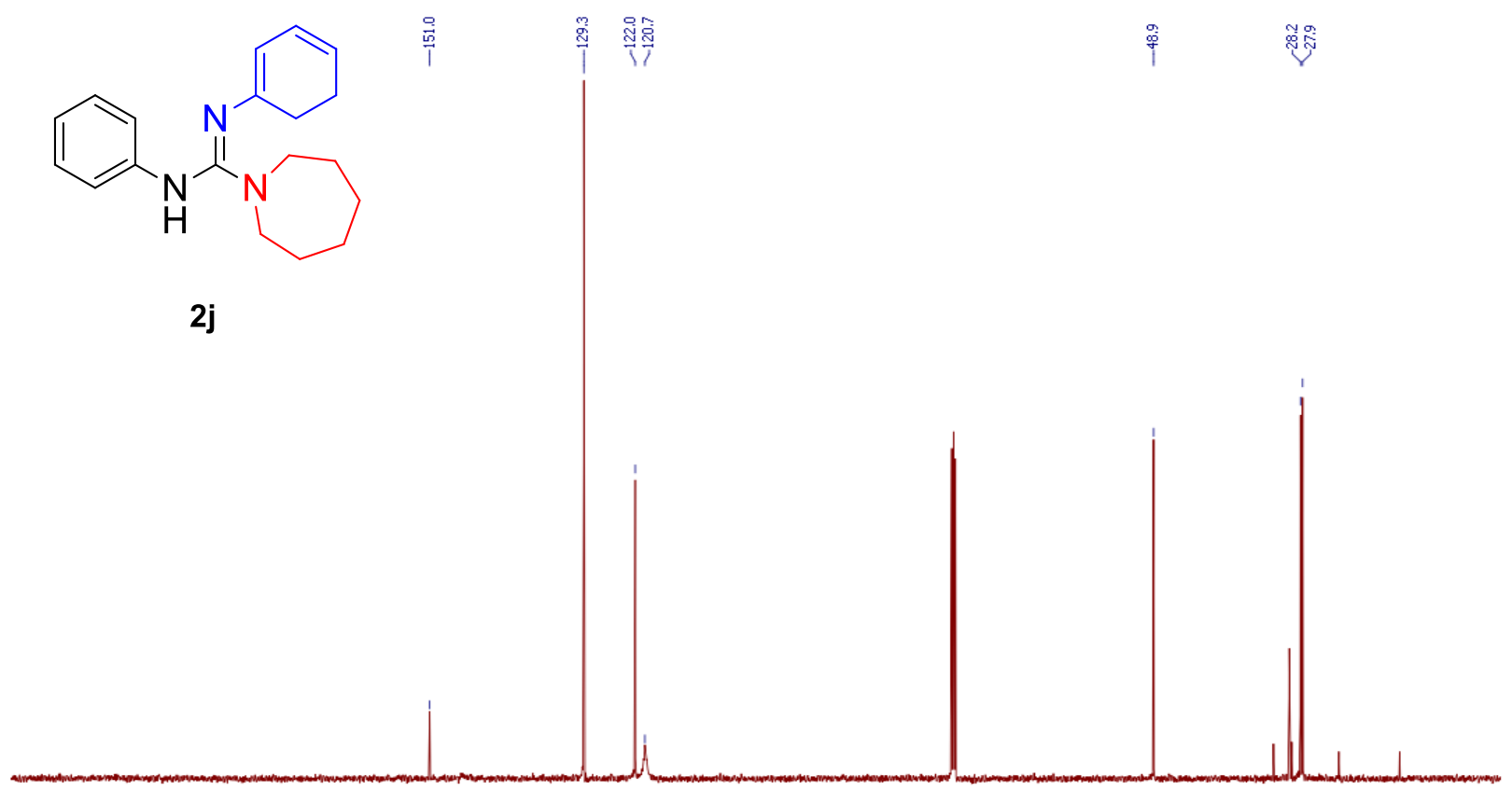

Figure S22. ${ }^{13} \mathrm{C}$ NMR spectrum of $\mathbf{2 j}\left(\mathbf{1 2 5} \mathrm{MHz}, \mathrm{CDCl}_{3}\right)$ 

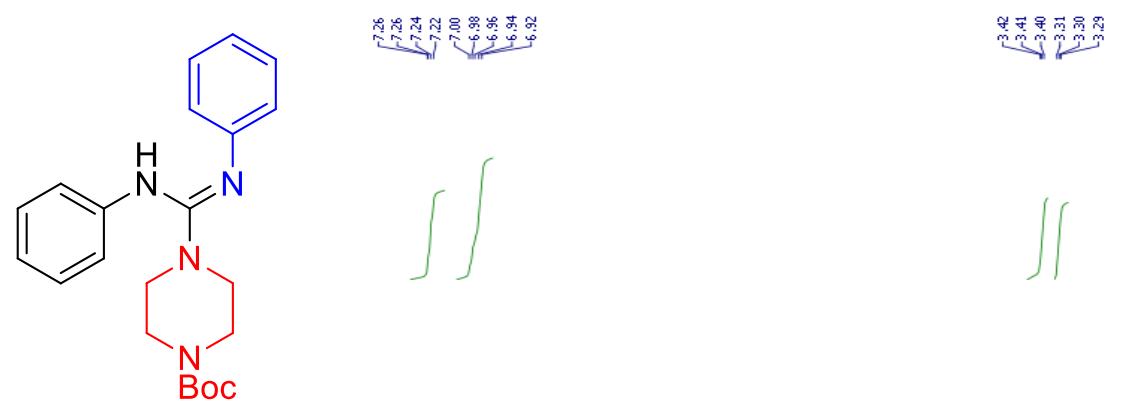

2k

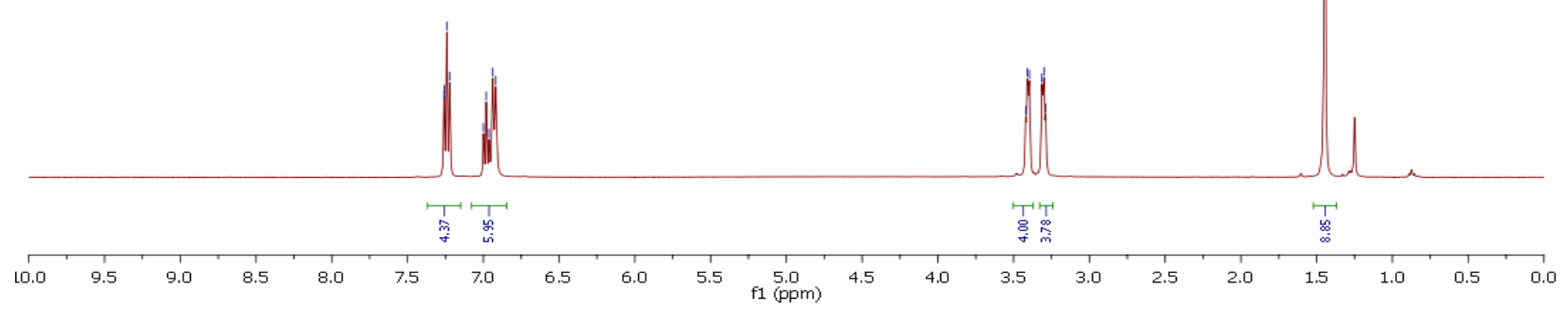

Figure S23. ${ }^{1} \mathrm{H}$ NMR spectrum of $2 \mathrm{k}\left(400 \mathrm{MHz}, \mathrm{CDCl}_{3}\right)$
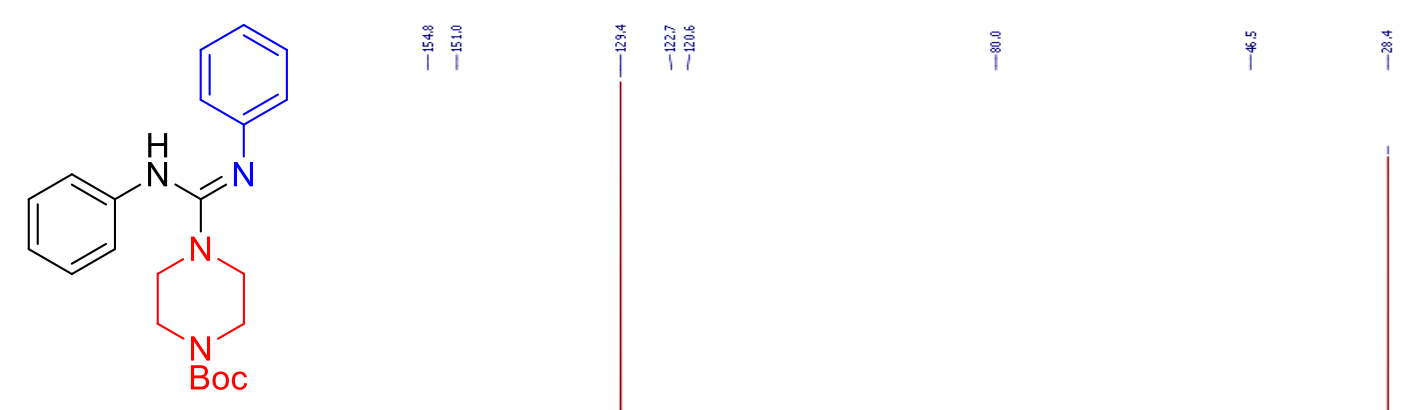

$2 k$
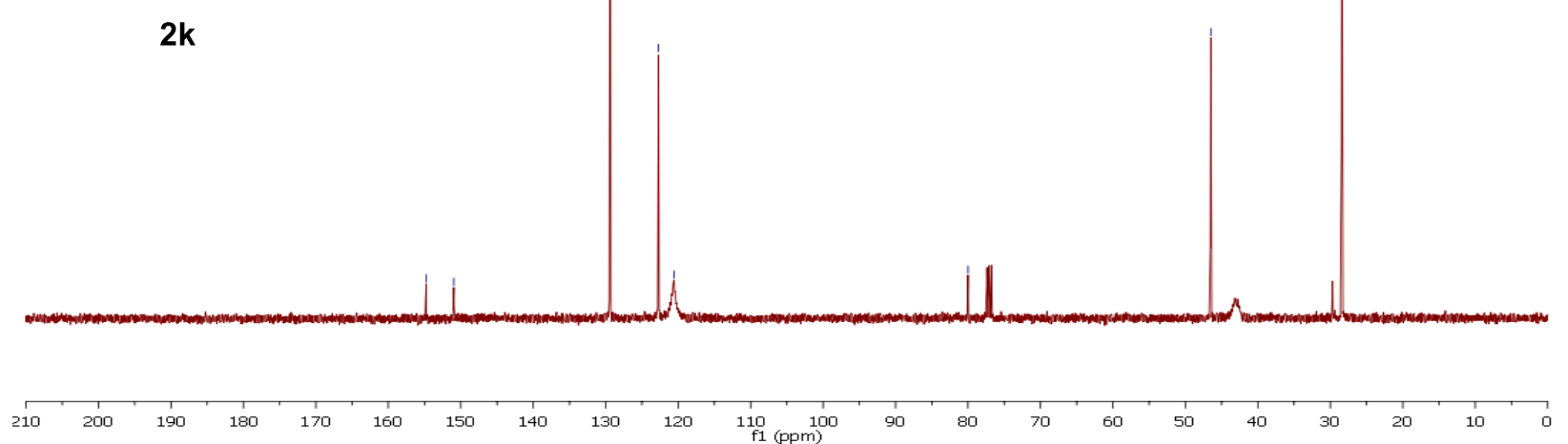

Figure S24. ${ }^{13} \mathrm{C}$ NMR spectrum of $\mathbf{2 k}\left(\mathbf{1 0 0} \mathbf{~ M H z}, \mathbf{C D C l}_{3}\right)$ 


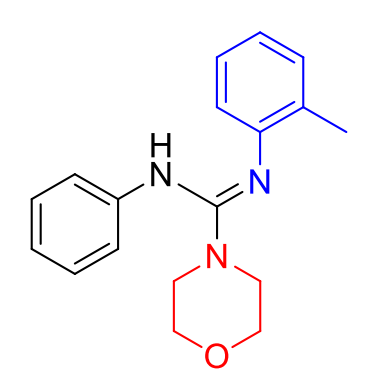

3b
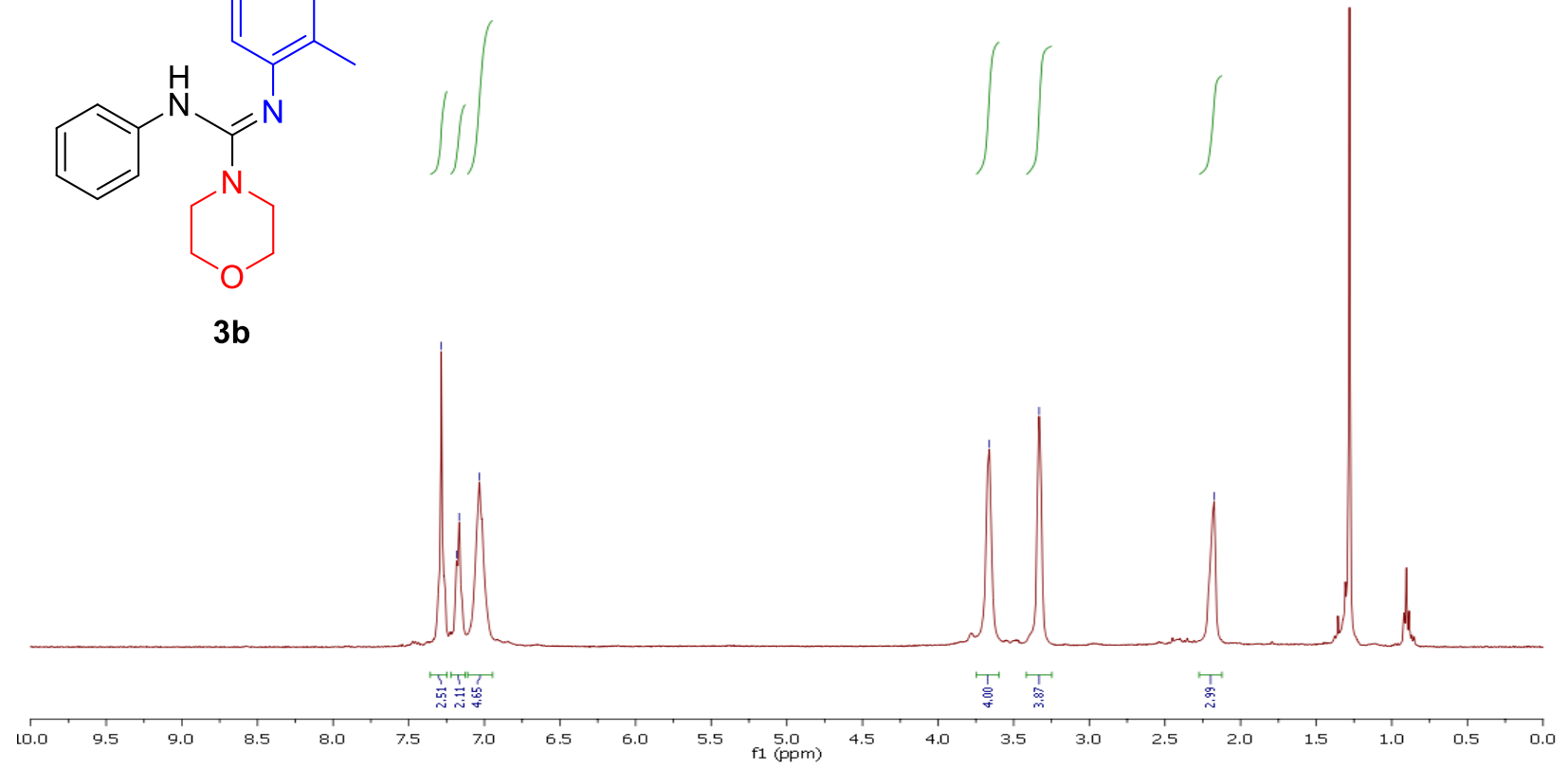

Figure S25. ${ }^{1} \mathrm{H}$ NMR spectrum of $3 \mathrm{~b}\left(400 \mathrm{MHz}, \mathrm{CDCl}_{3}\right)$<smiles>Cc1ccccc1/N=C(\Nc1ccccc1)N1CCOCC1</smiles>

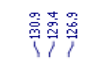

3b

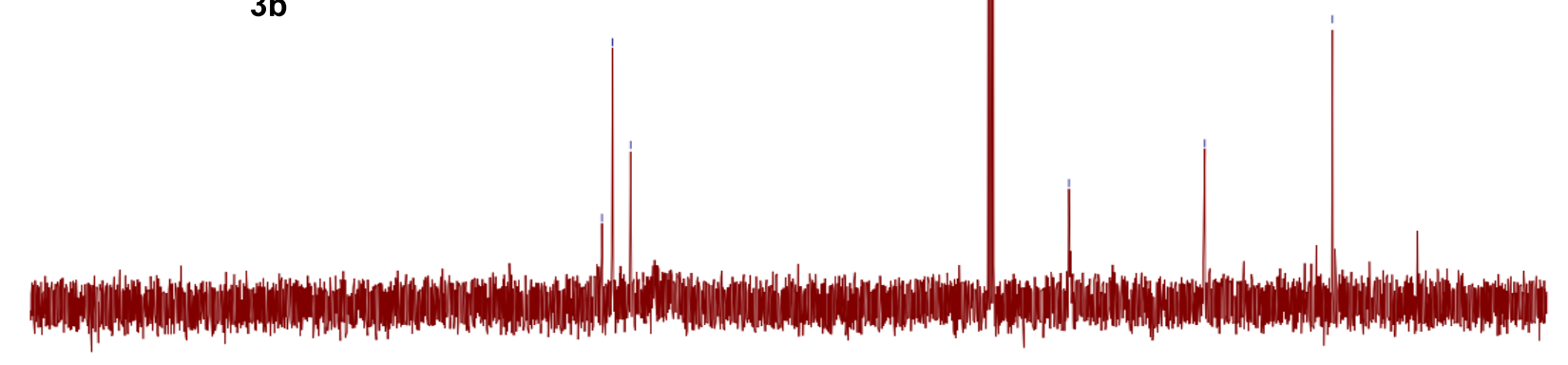

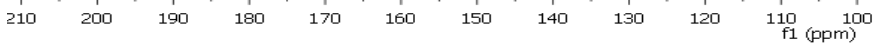

Figure S26. ${ }^{13} \mathrm{C}$ NMR spectrum of $\mathbf{3 b}\left(\mathbf{1 0 0} \mathrm{MHz}, \mathrm{CDCl}_{3}\right)$ 


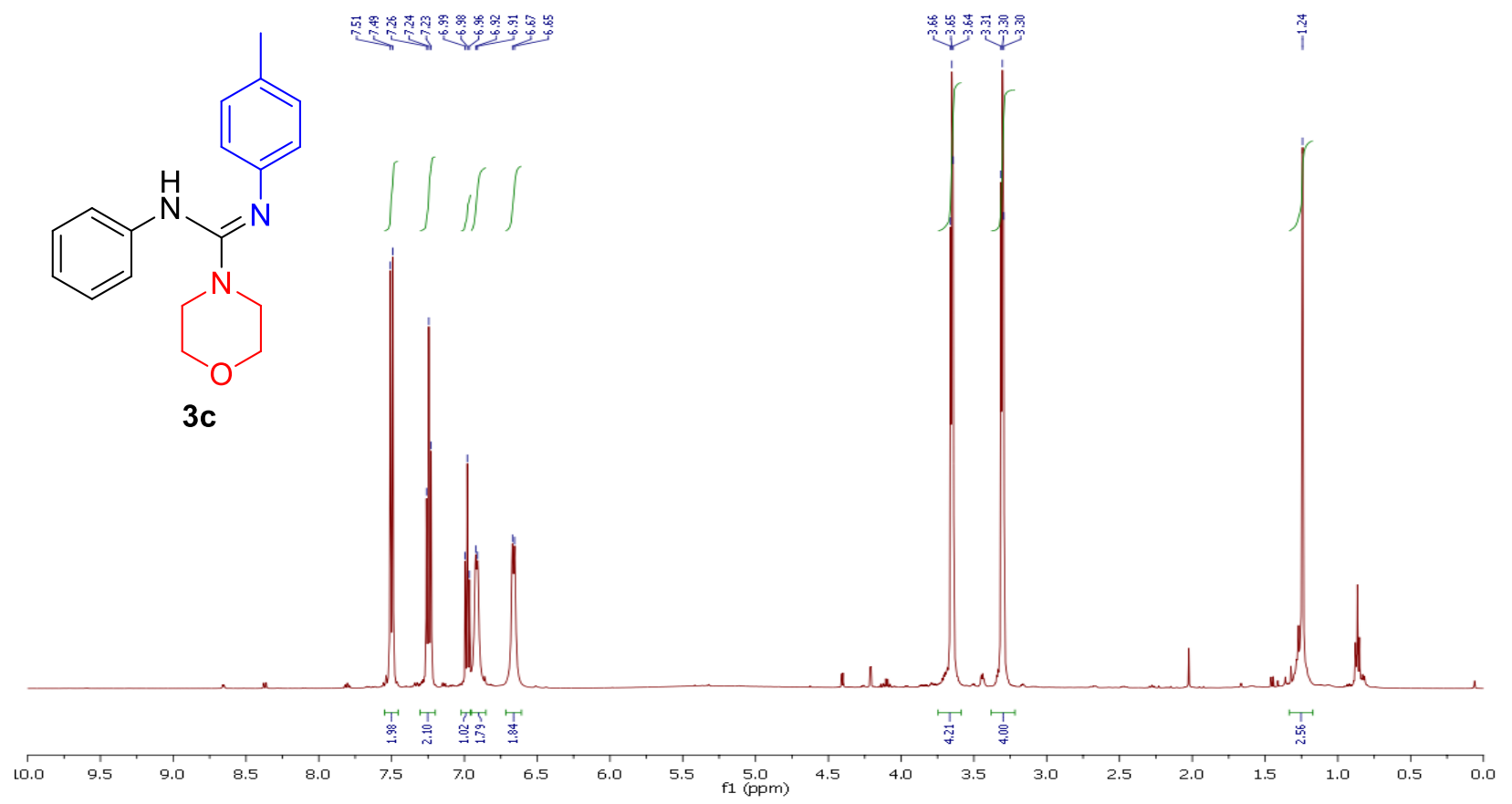

Figure S27. ${ }^{1} \mathrm{H}$ NMR spectrum of $\mathbf{3 c}\left(\mathbf{5 0 0} \mathrm{MHz}, \mathrm{CDCl}_{3}\right)$

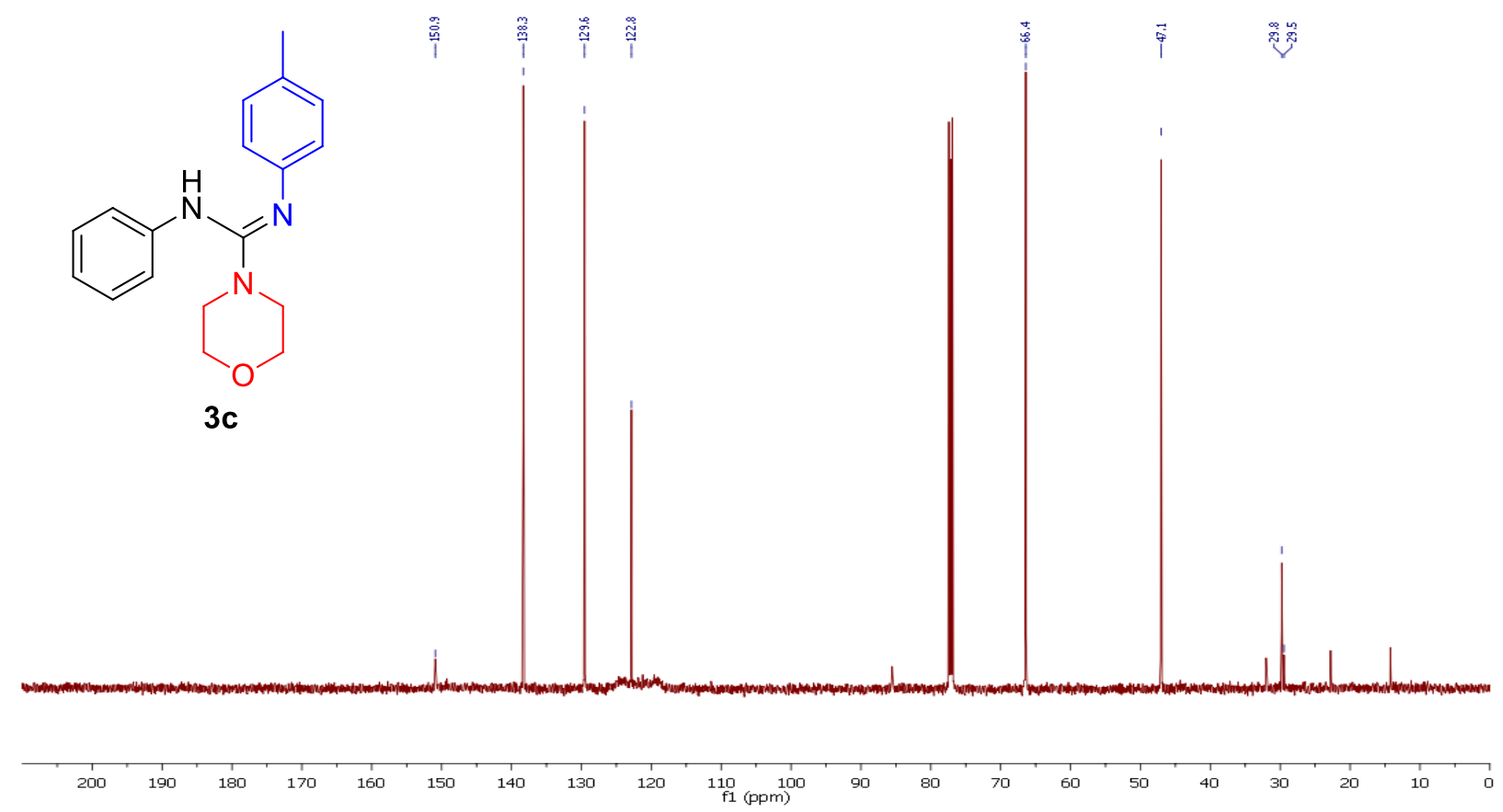

Figure S28. ${ }^{13} \mathrm{C}$ NMR spectrum of $\mathbf{3 c}\left(\mathbf{1 2 5} \mathrm{MHz}, \mathrm{CDCl}_{3}\right)$ 


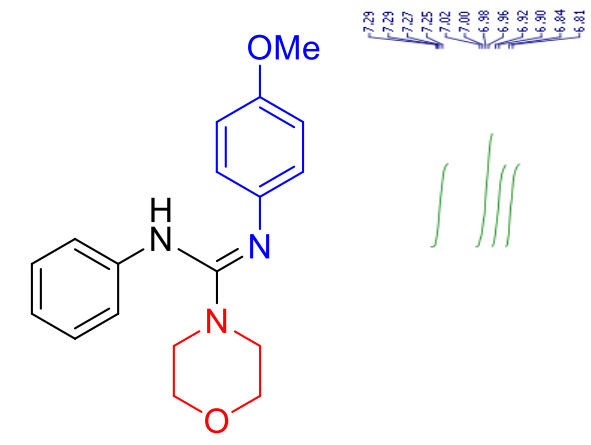

3d

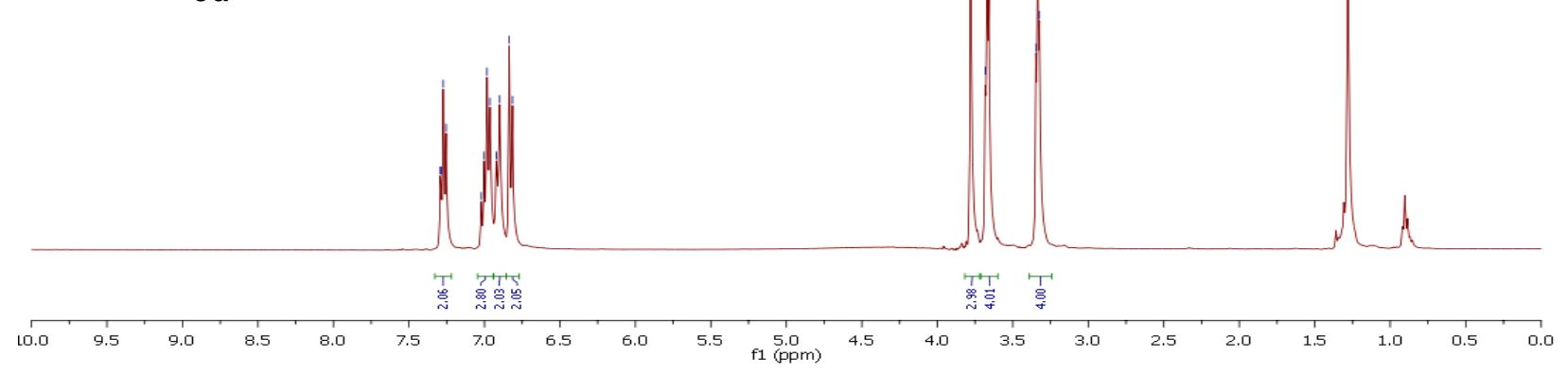

Figure S29. ${ }^{1} \mathrm{H}$ NMR spectrum of $\mathbf{3 d}\left(400 \mathrm{MHz}, \mathrm{CDCl}_{3}\right)$

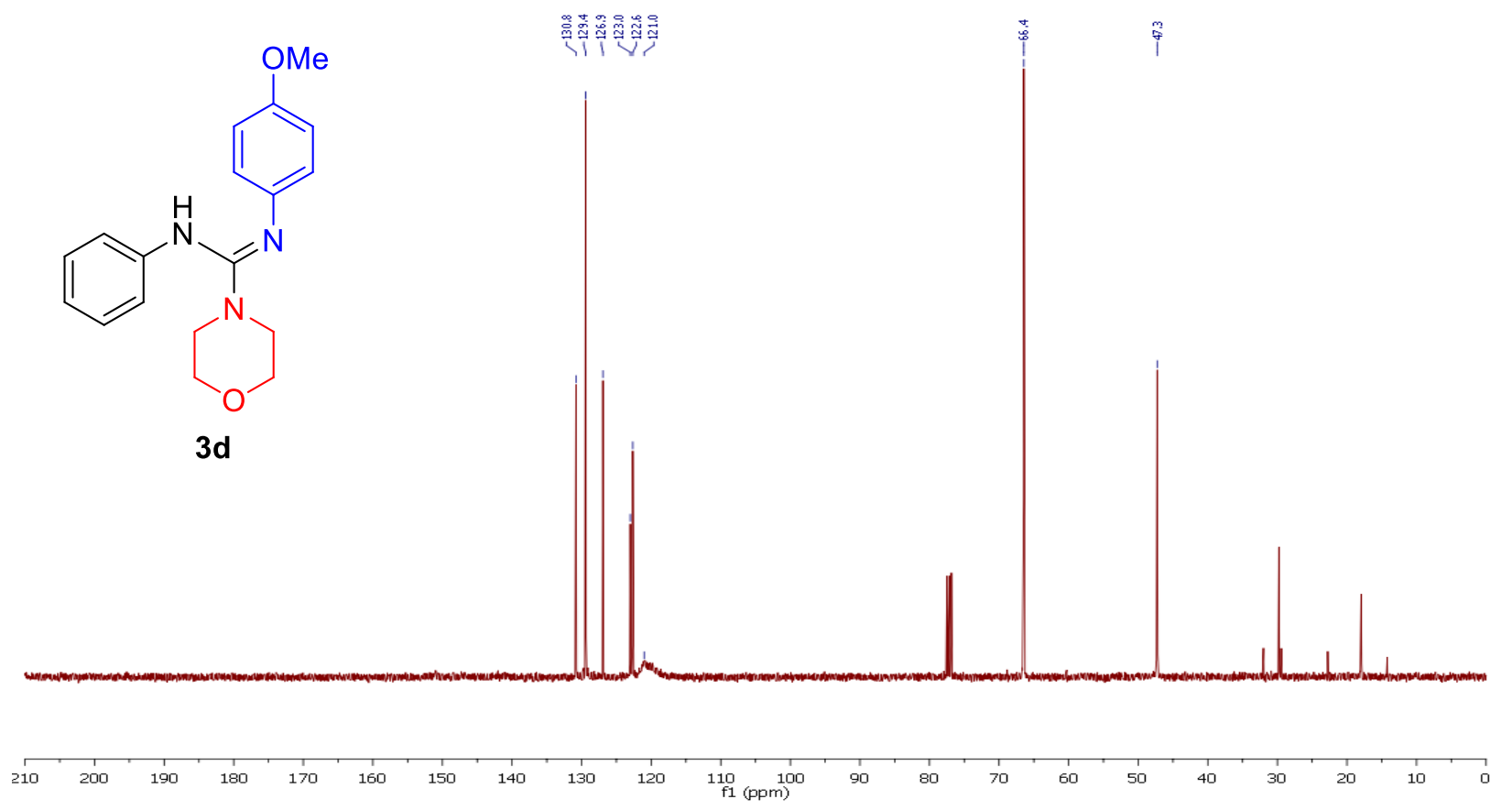

Figure S30. ${ }^{13} \mathrm{C}$ NMR spectrum of $\mathbf{3 d}\left(\mathbf{1 2 5} \mathrm{MHz}, \mathrm{CDCl}_{3}\right)$ 


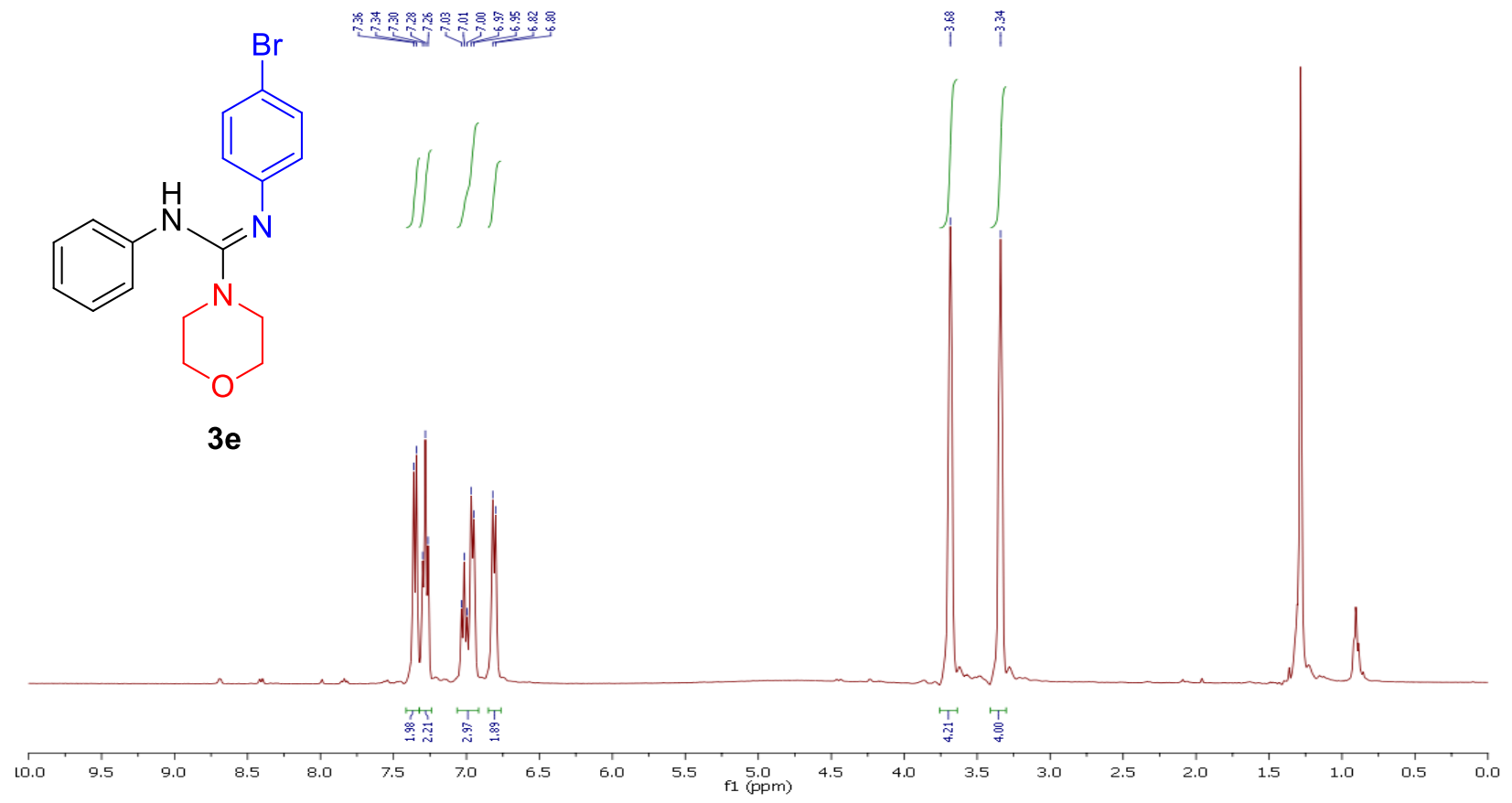

Figure S31. ${ }^{1} \mathrm{H}$ NMR spectrum of $3 \mathrm{e}\left(400 \mathrm{MHz}, \mathrm{CDCl}_{3}\right)$

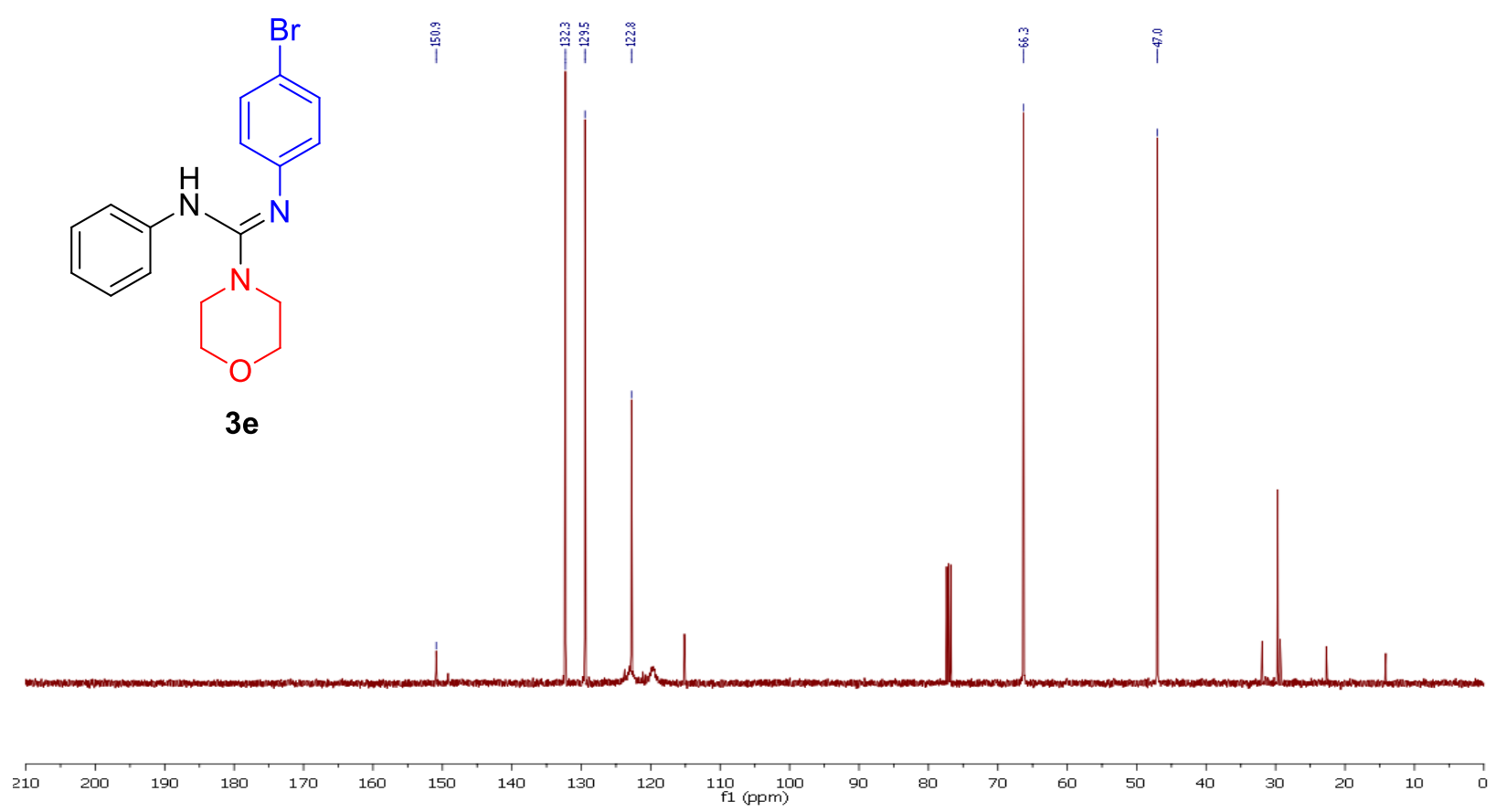

Figure S32. ${ }^{13} \mathrm{C}$ NMR spectrum of $\mathbf{3 e}\left(\mathbf{1 0 0} \mathbf{M H z}, \mathbf{C D C l}_{3}\right)$ 


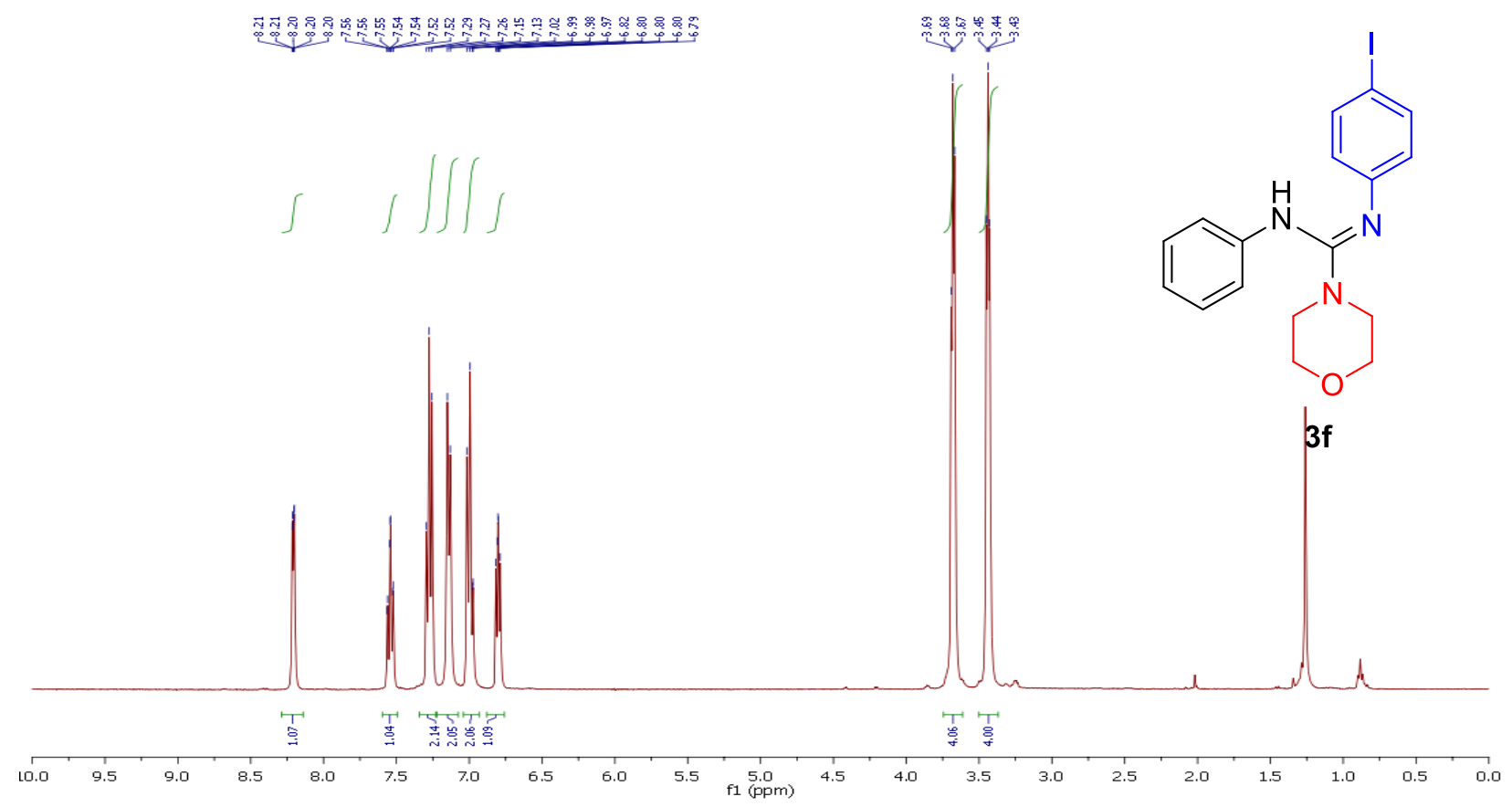

Figure S33. ${ }^{1} \mathrm{H}$ NMR spectrum of $3 f\left(400 \mathrm{MHz}, \mathrm{CDCl}_{3}\right)$
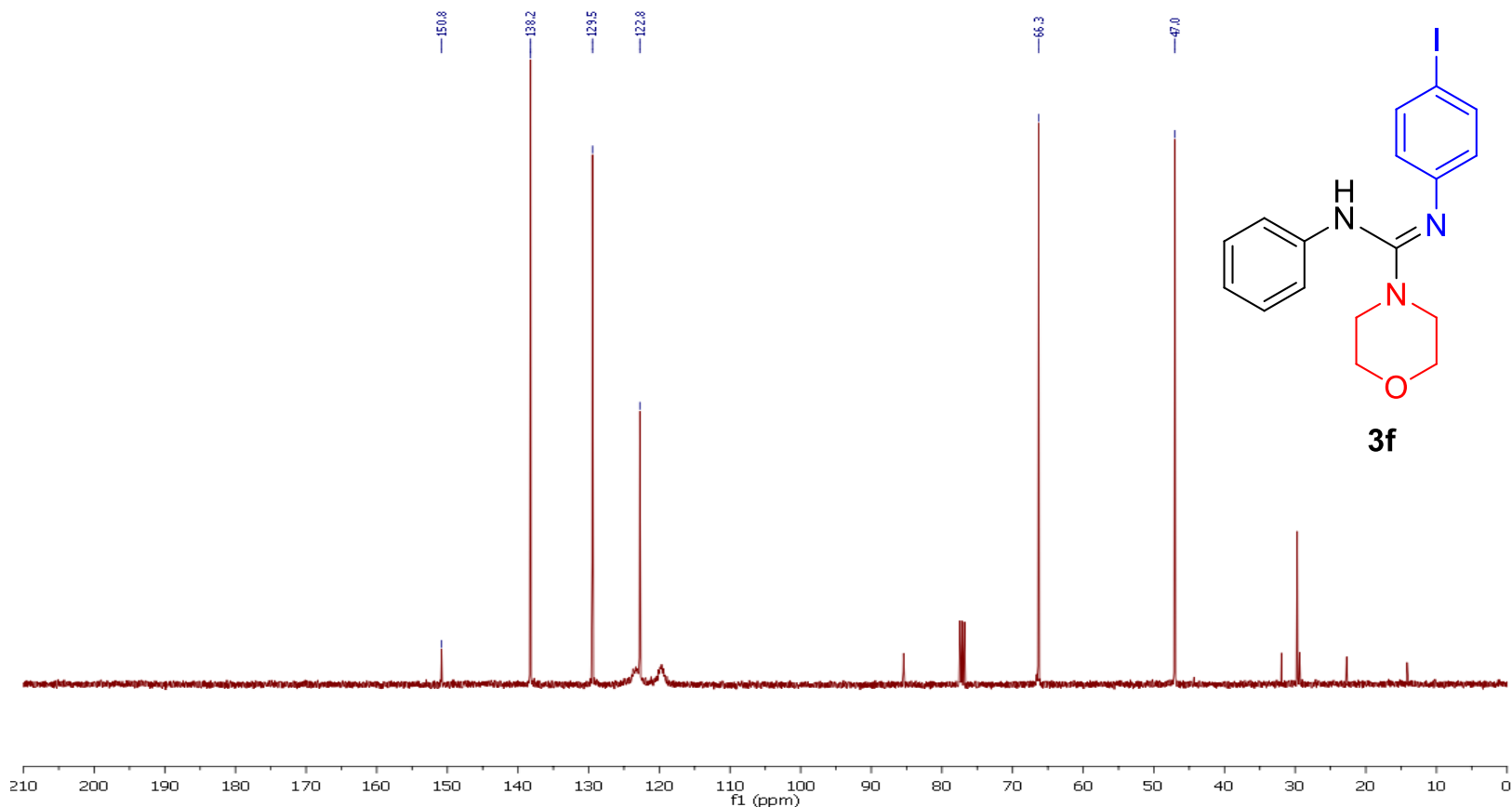

Figure S34. ${ }^{13} \mathrm{C}$ NMR spectrum of $\mathbf{3 f}\left(\mathbf{1 0 0} \mathrm{MHz}, \mathrm{CDCl}_{3}\right)$ 


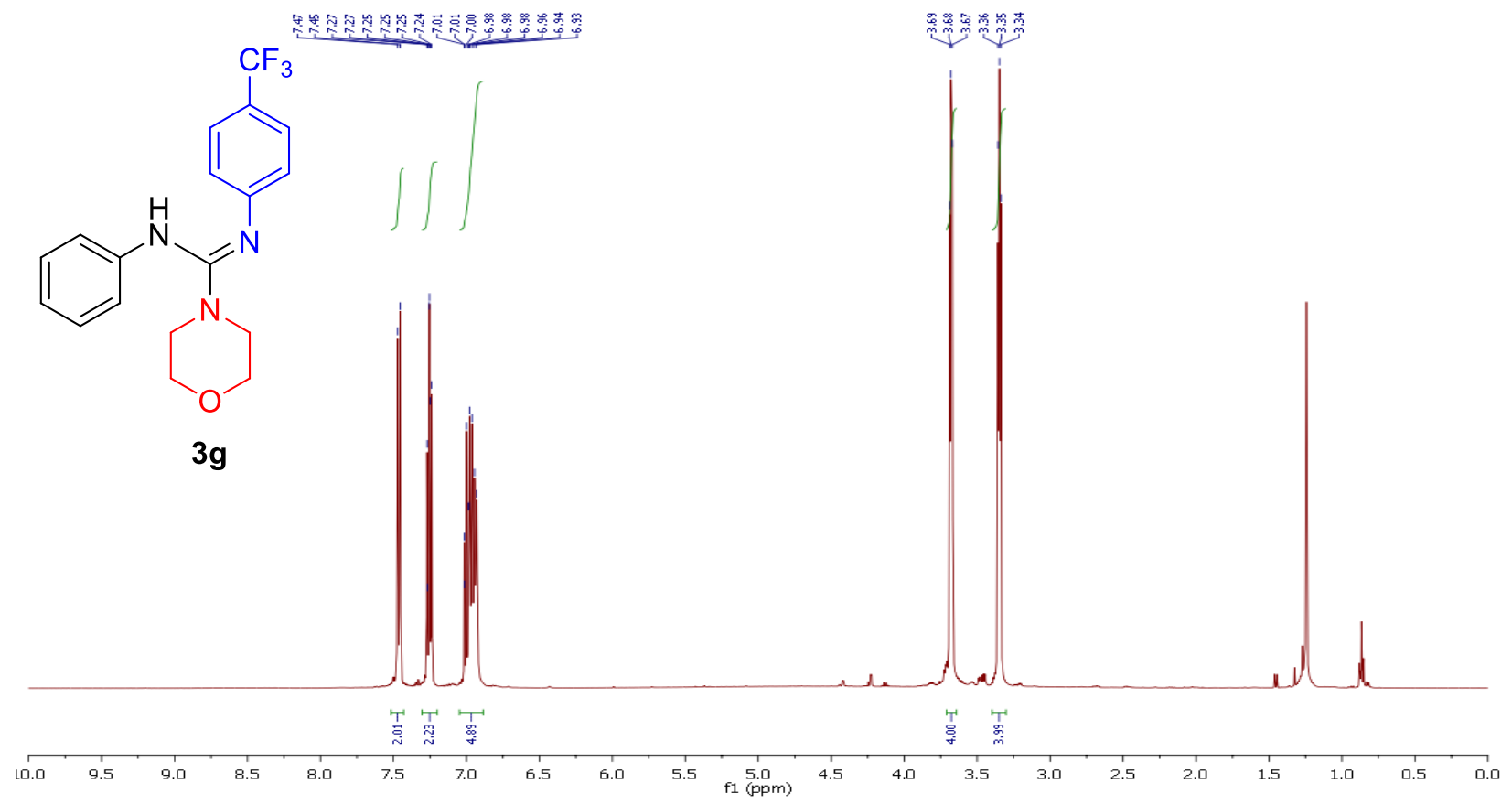

Figure S35. ${ }^{1} \mathrm{H}$ NMR spectrum of $3 \mathrm{~g}(500 \mathrm{MHz}, \mathbf{C D C l})$

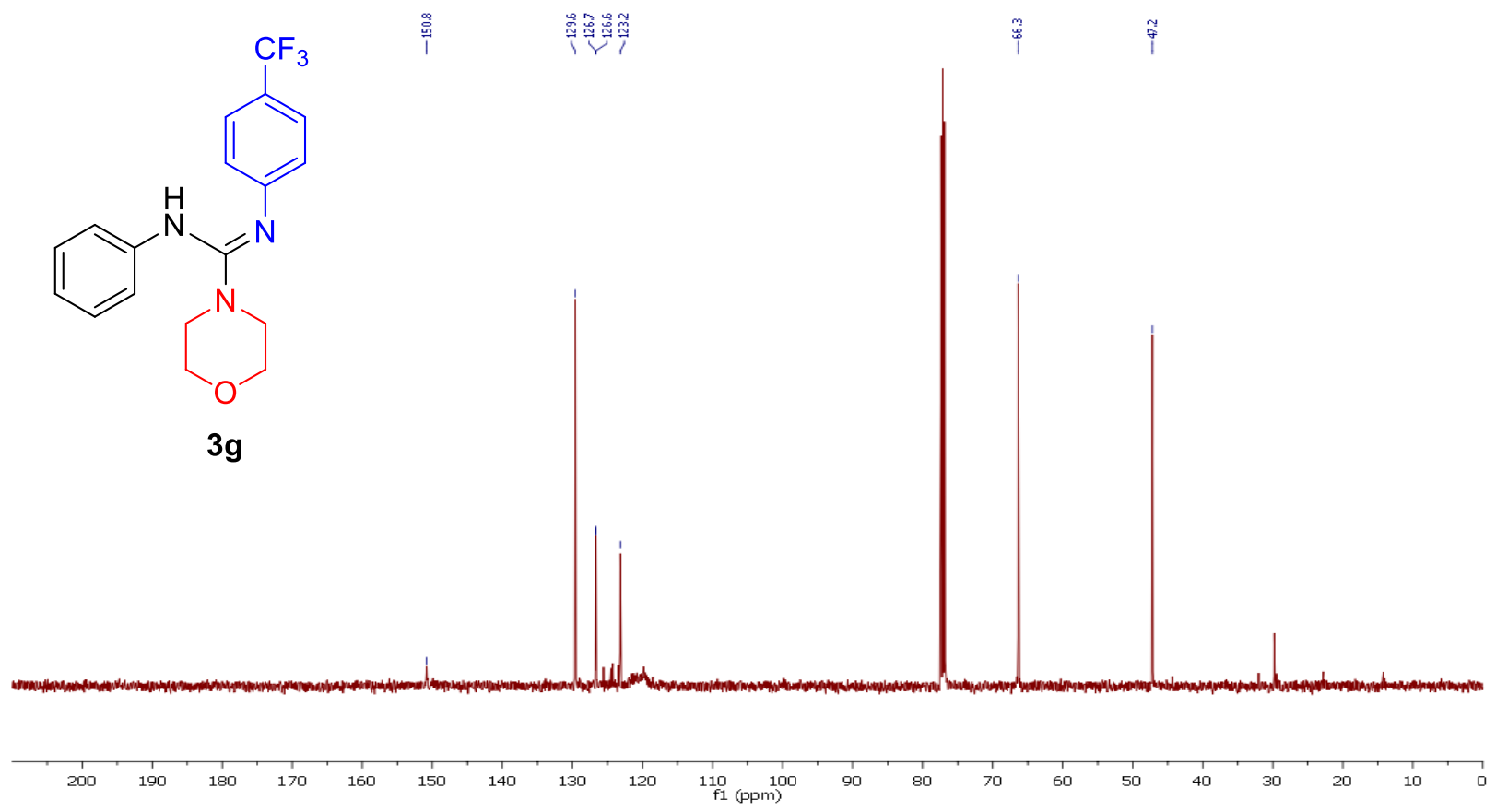

Figure S36. ${ }^{13} \mathrm{C}$ NMR spectrum of $3 \mathrm{~g}\left(\mathbf{1 2 5} \mathrm{MHz}, \mathrm{CDCl}_{3}\right)$ 

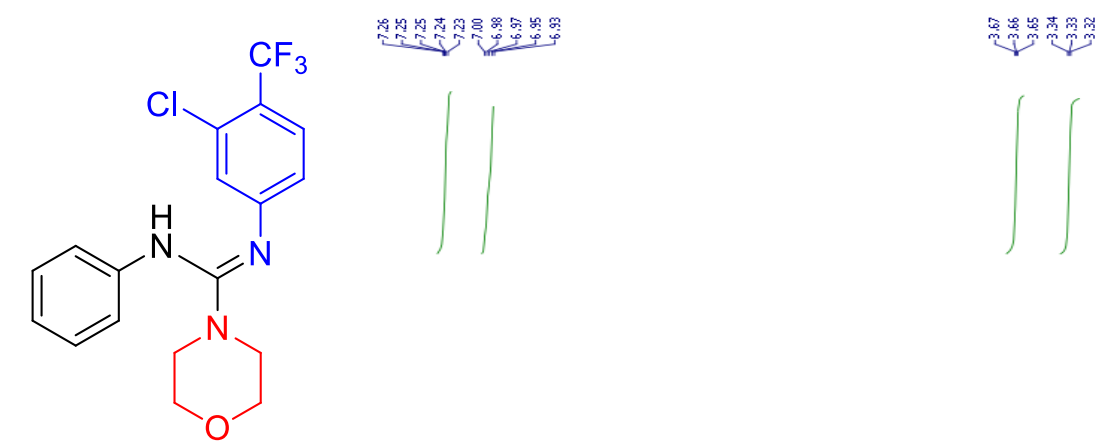

3h
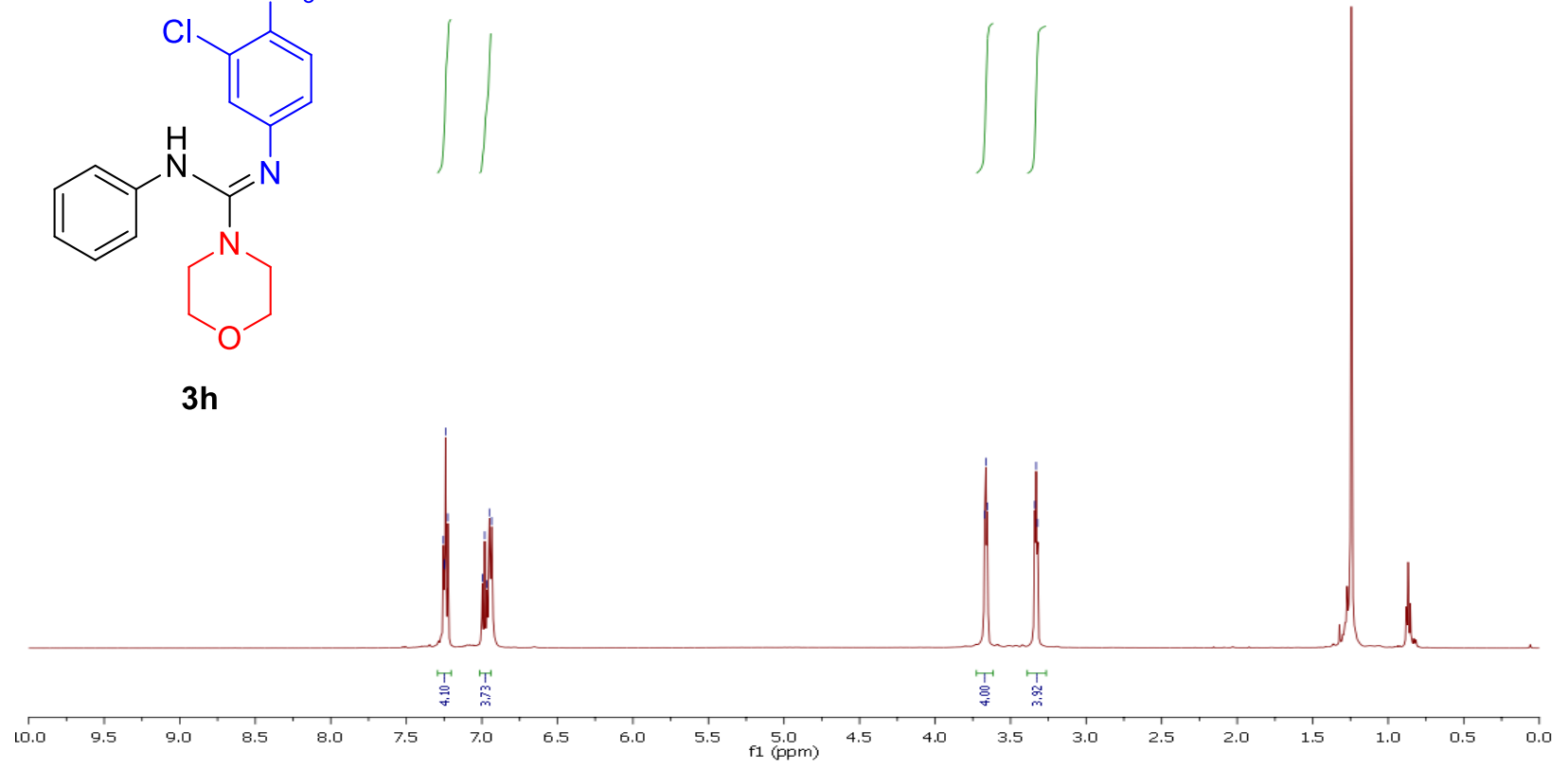

Figure S37. ${ }^{1} \mathrm{H}$ NMR spectrum of 3 h (500 MHz, CDCl3)

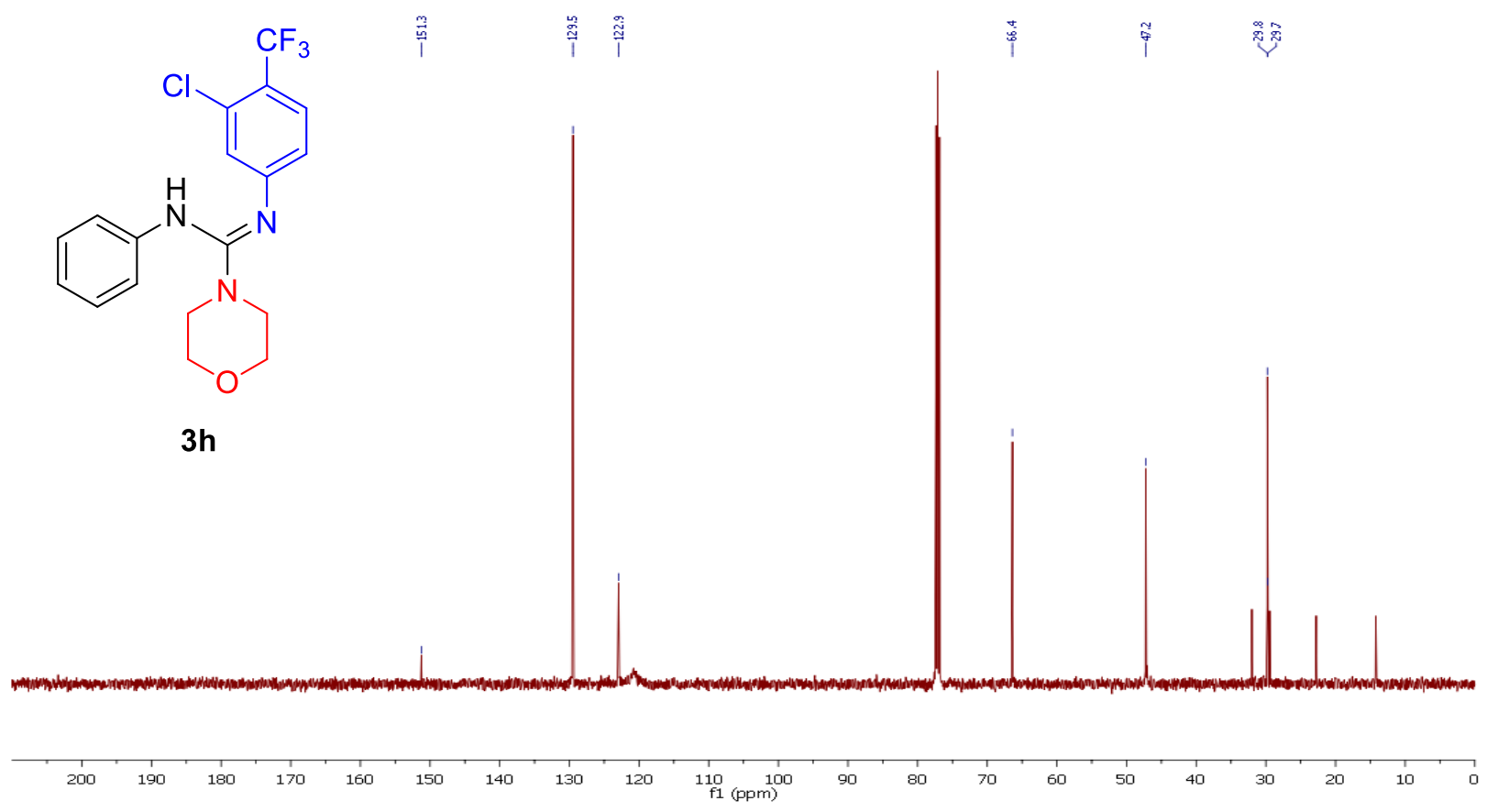

Figure S38. ${ }^{13} \mathrm{C}$ NMR spectrum of $3 \mathrm{~h}\left(\mathbf{1 2 5} \mathrm{MHz}, \mathrm{CDCl}_{3}\right)$ 


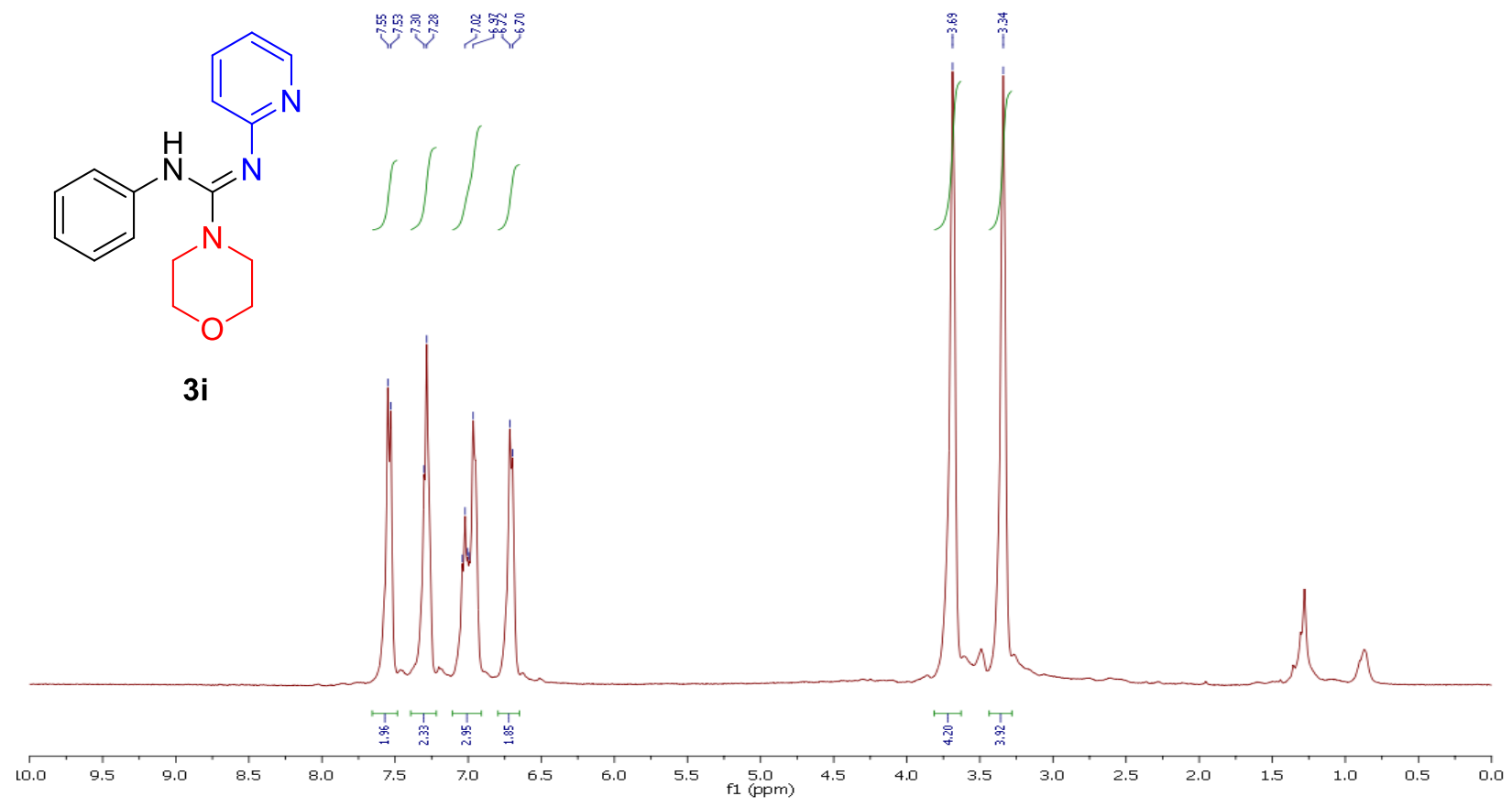

Figure S39. ${ }^{1} \mathrm{H}$ NMR spectrum of $3 \mathbf{i}\left(400 \mathrm{MHz}, \mathrm{CDCl}_{3}\right)$<smiles>c1ccc(N/C(=N/c2ccccn2)N2CCOCC2)cc1</smiles>

$3 \mathbf{i}$
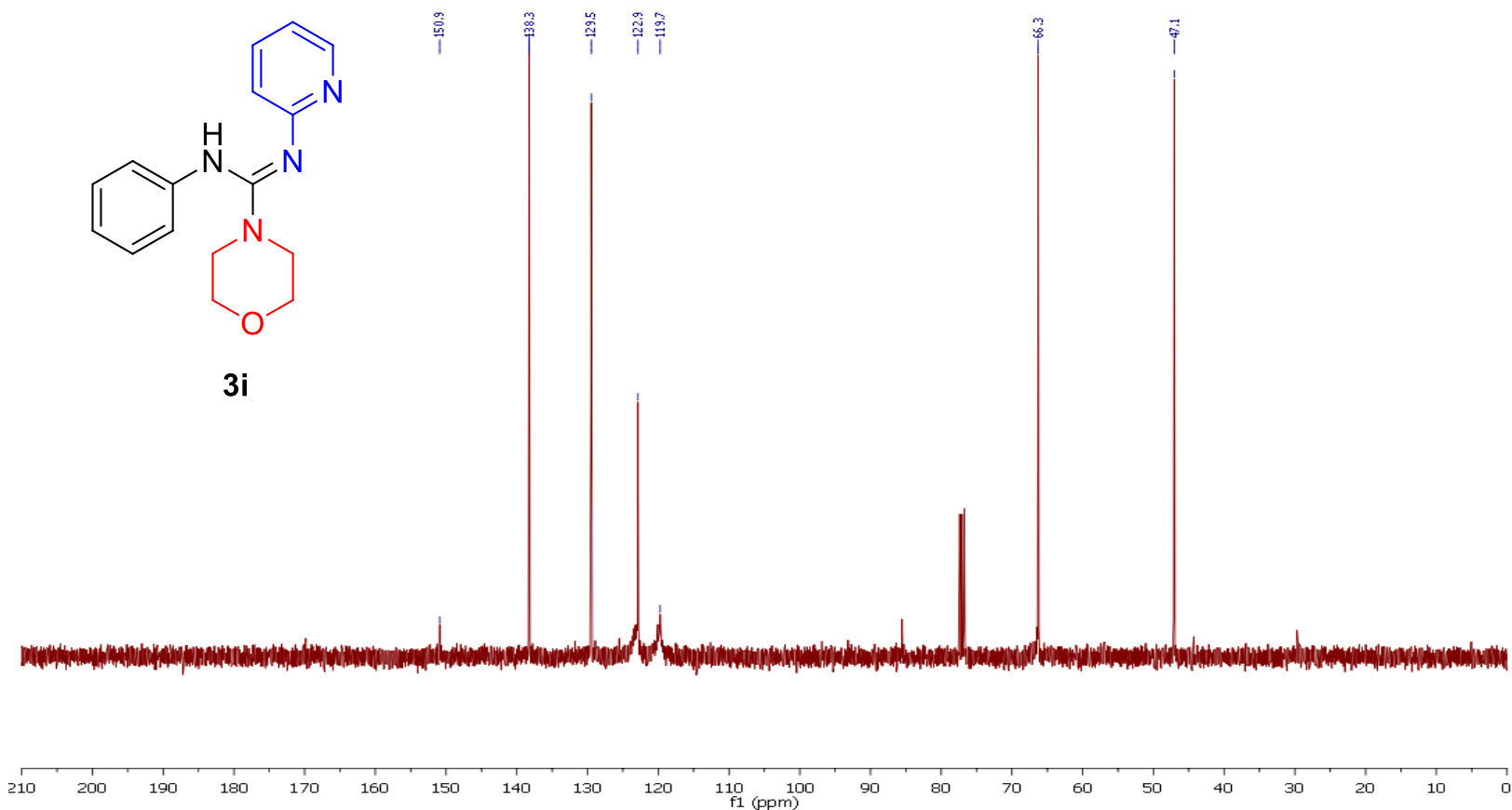

Figure S40. ${ }^{13} \mathrm{C}$ NMR spectrum of $3 \mathbf{i}\left(100 \mathrm{MHz}, \mathrm{CDCl}_{3}\right)$ 


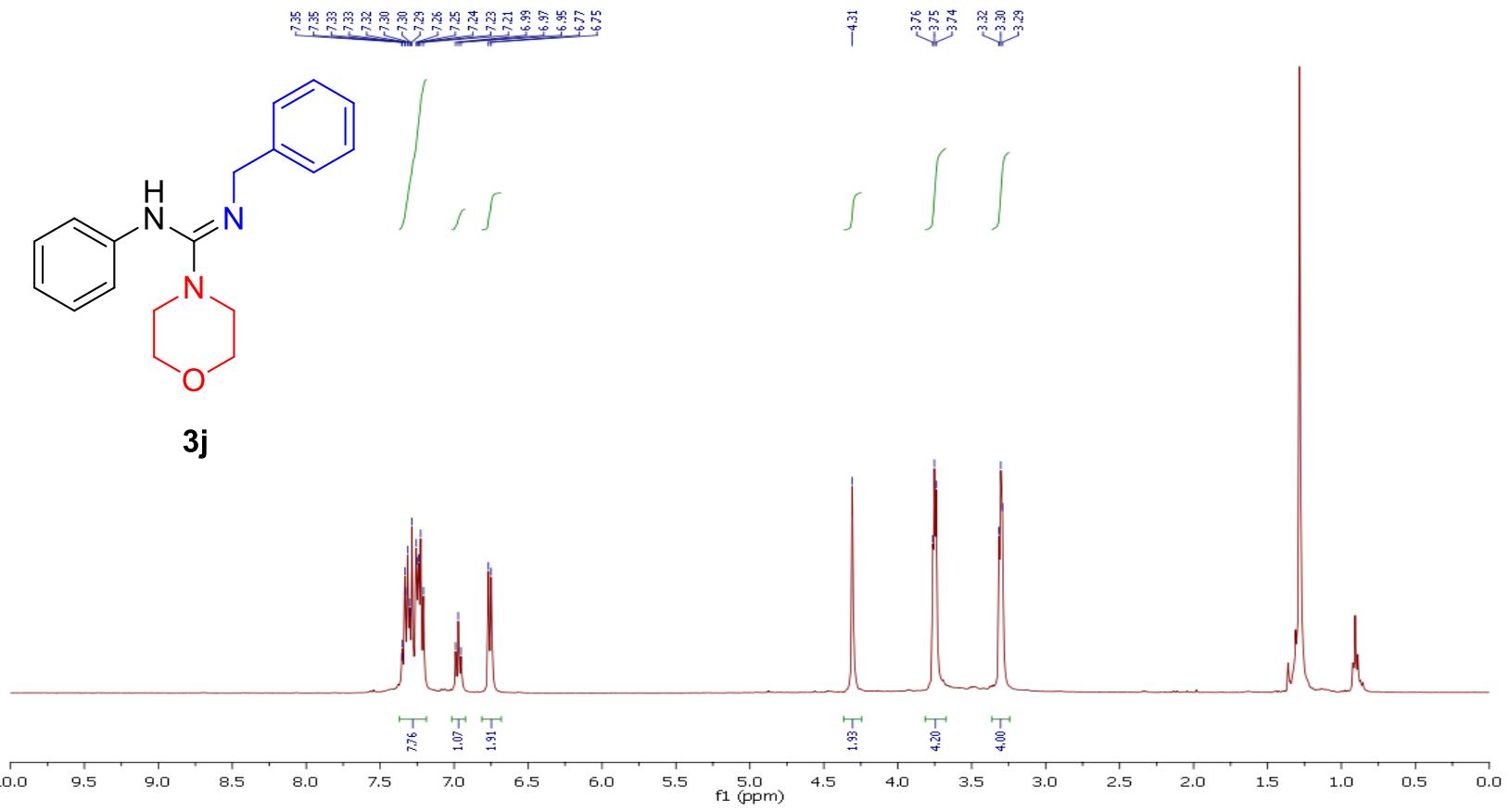

Figure S41. ${ }^{1} \mathrm{H}$ NMR spectrum of $\mathbf{3 j}$ (400 $\left.\mathbf{M H z}, \mathbf{C D C l} 3\right)$

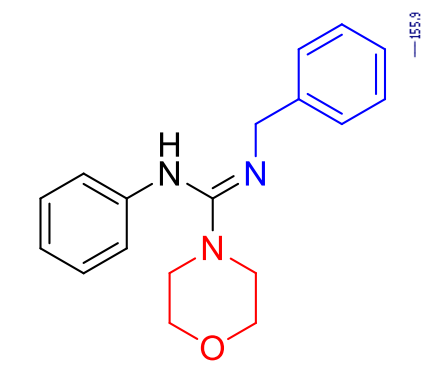

3j

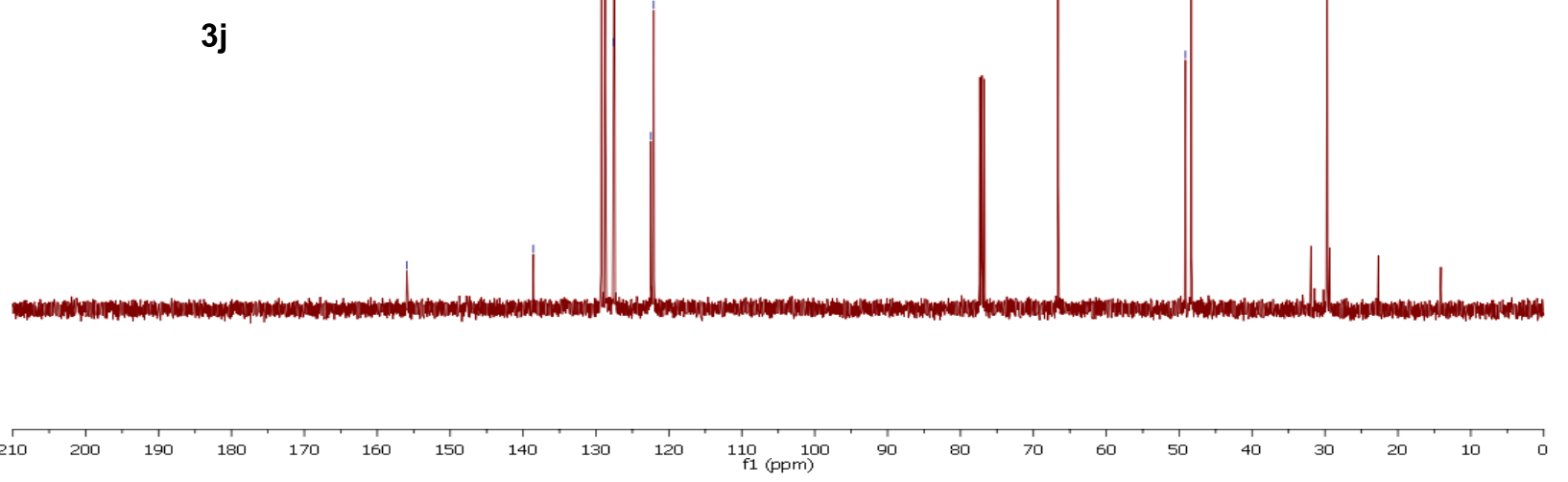

Figure S42. ${ }^{13} \mathrm{C}$ NMR spectrum of $\mathbf{3 j}\left(\mathbf{1 0 0} \mathbf{M H z} \mathbf{C D C l}_{3}\right)$ 


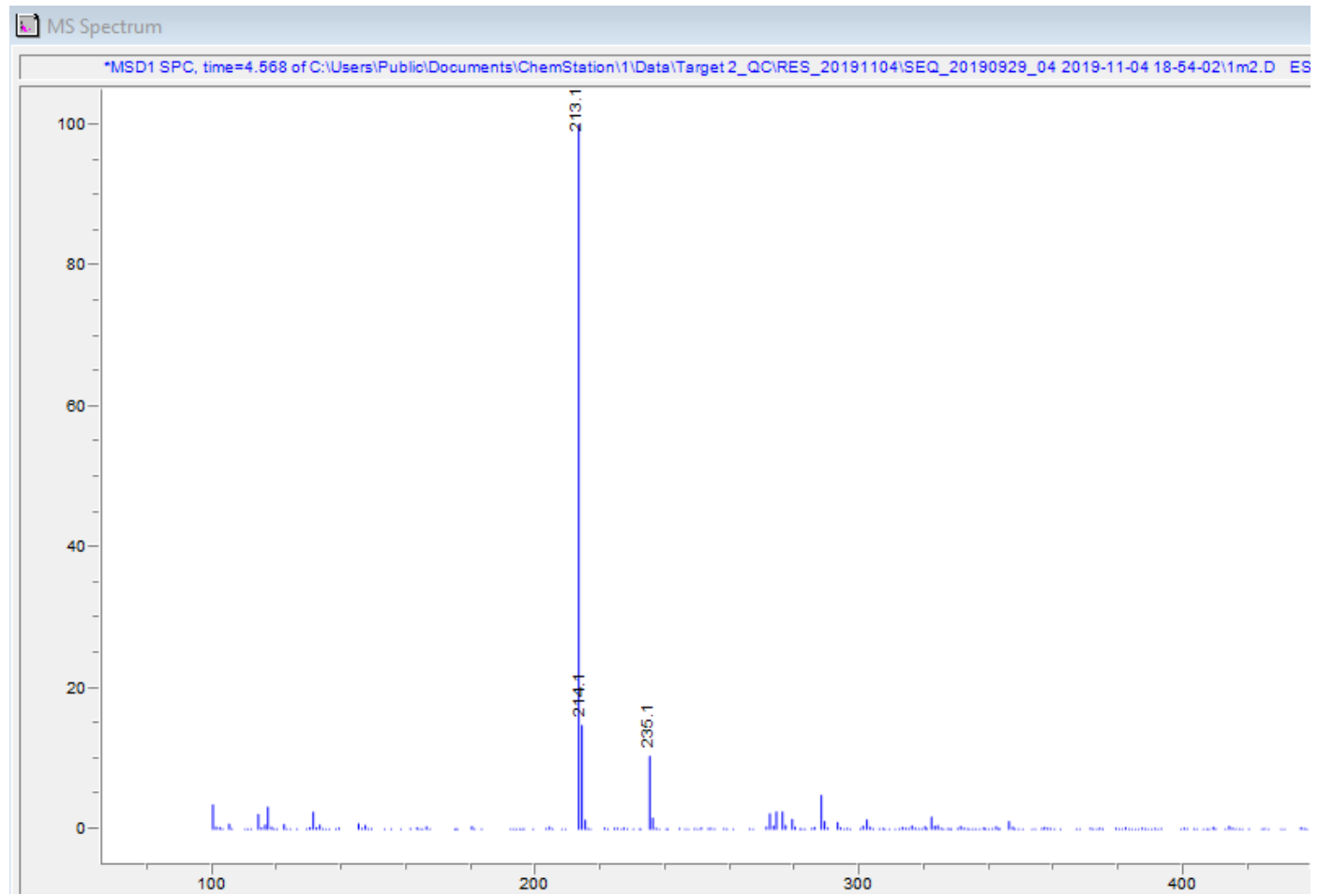

Figure S43. Mass spectrum of 5 


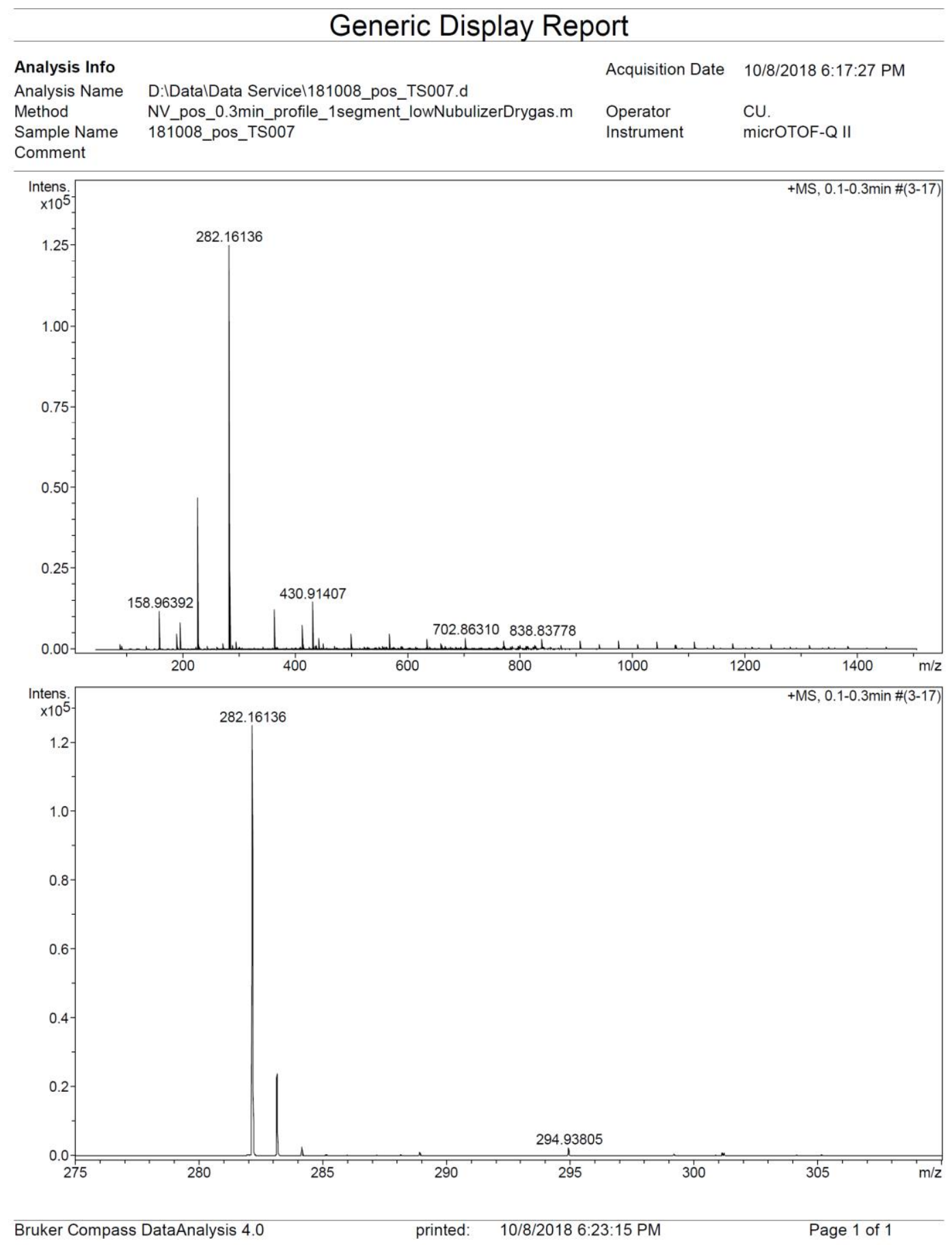

Figure S44. Mass spectrum of 2a 


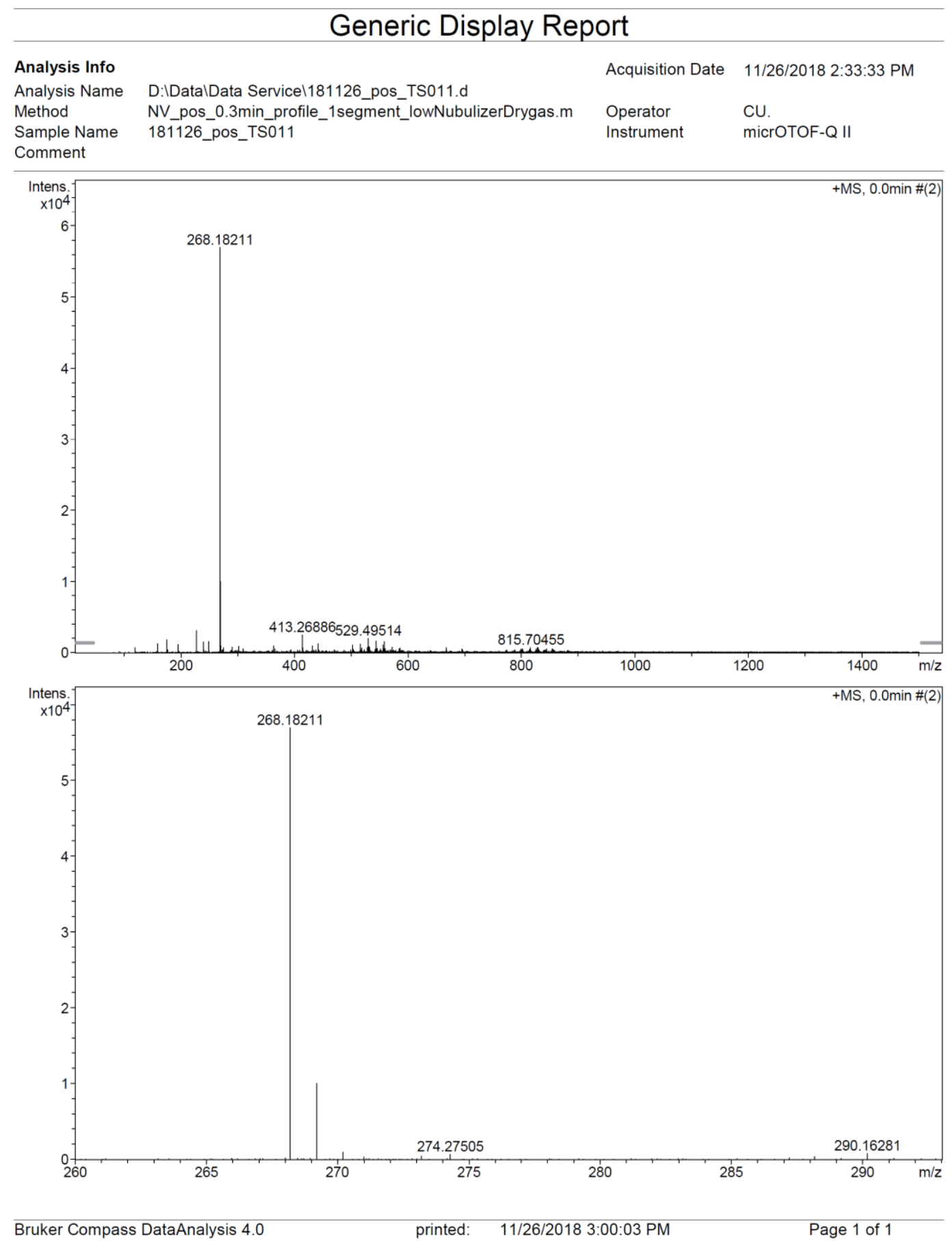

Figure S45. Mass spectrum of $\mathbf{2 b}$ 


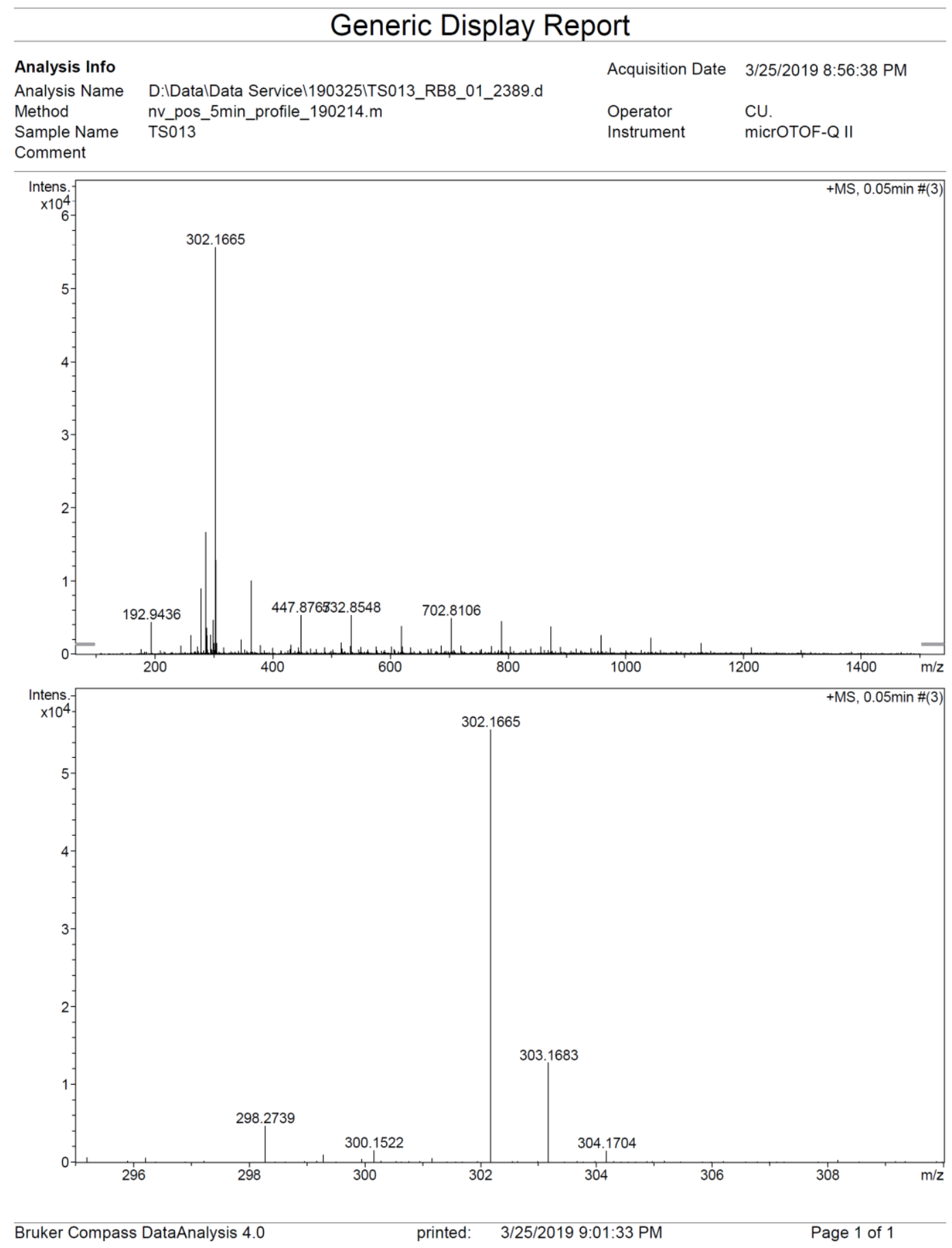

Figure S46. Mass spectrum of 2c 


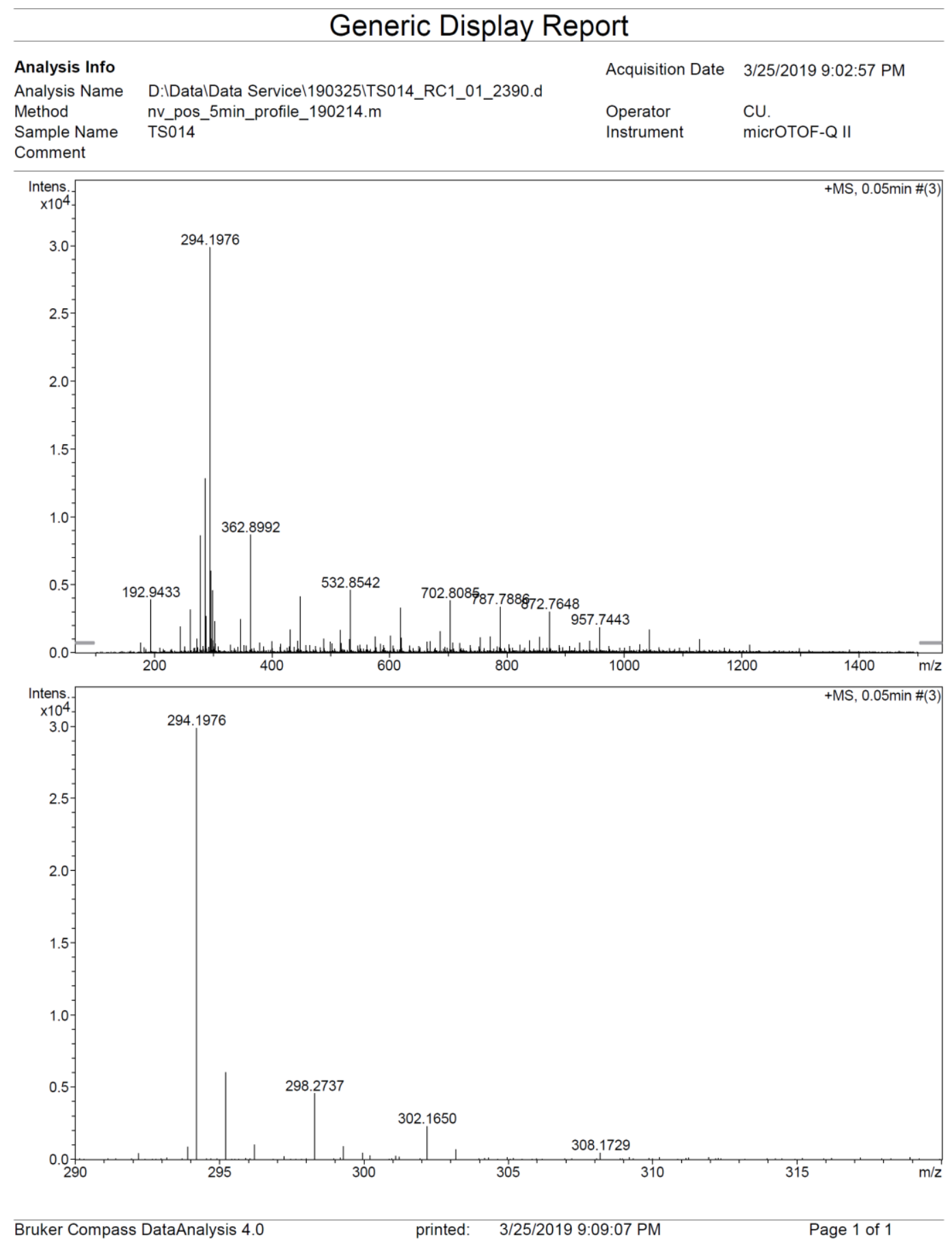

Figure S47. Mass spectrum of 2d 


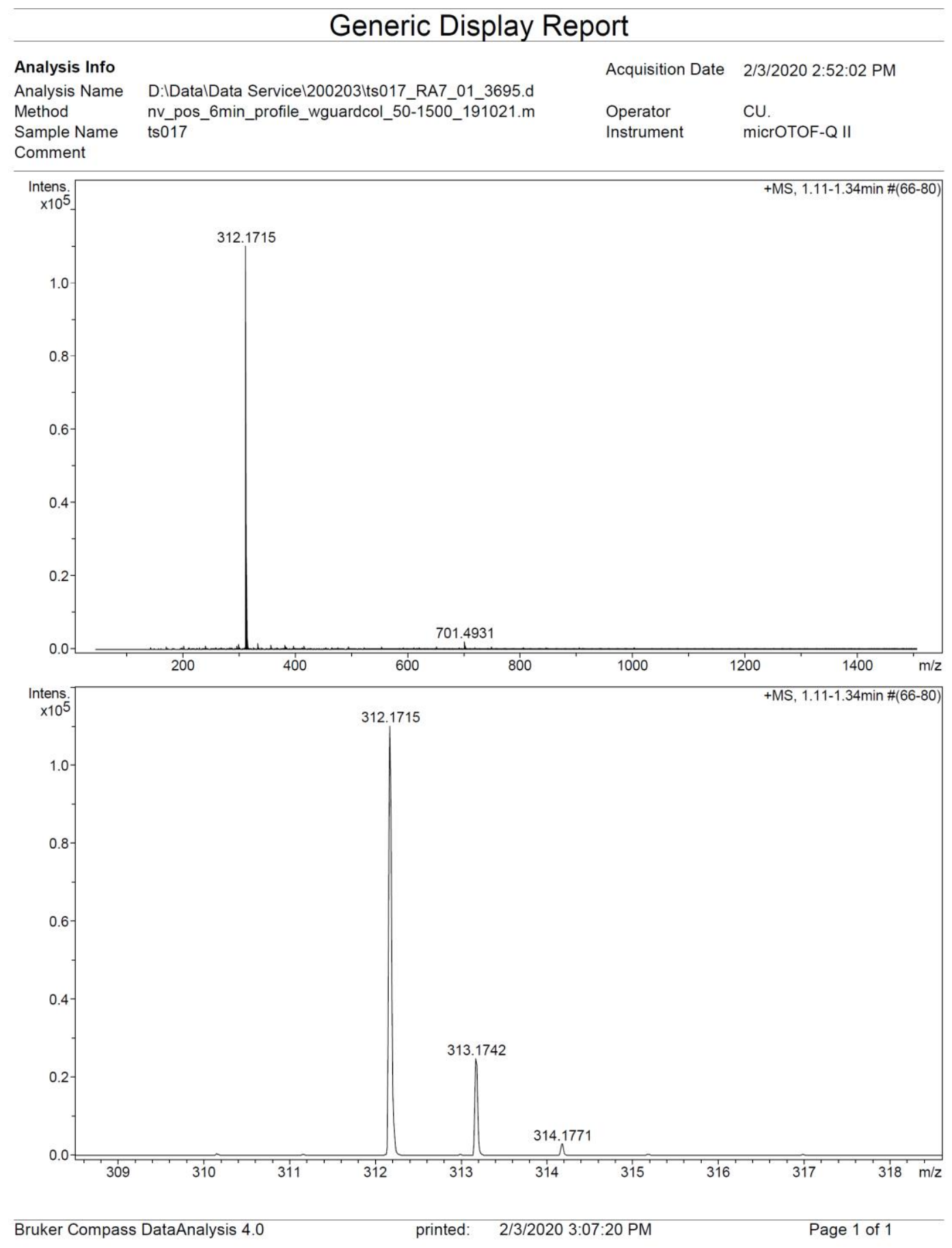

Figure S48. Mass spectrum of $2 \mathrm{e}$ 


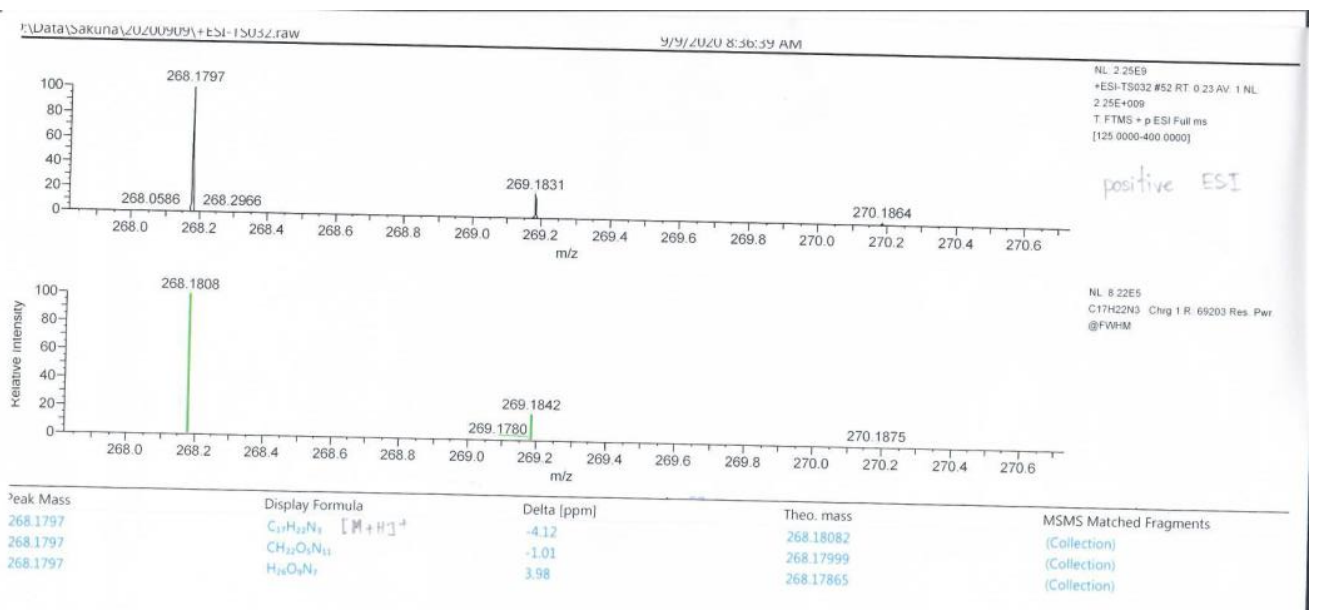

Figure S49. Mass spectrum of $\mathbf{2 g}$ 


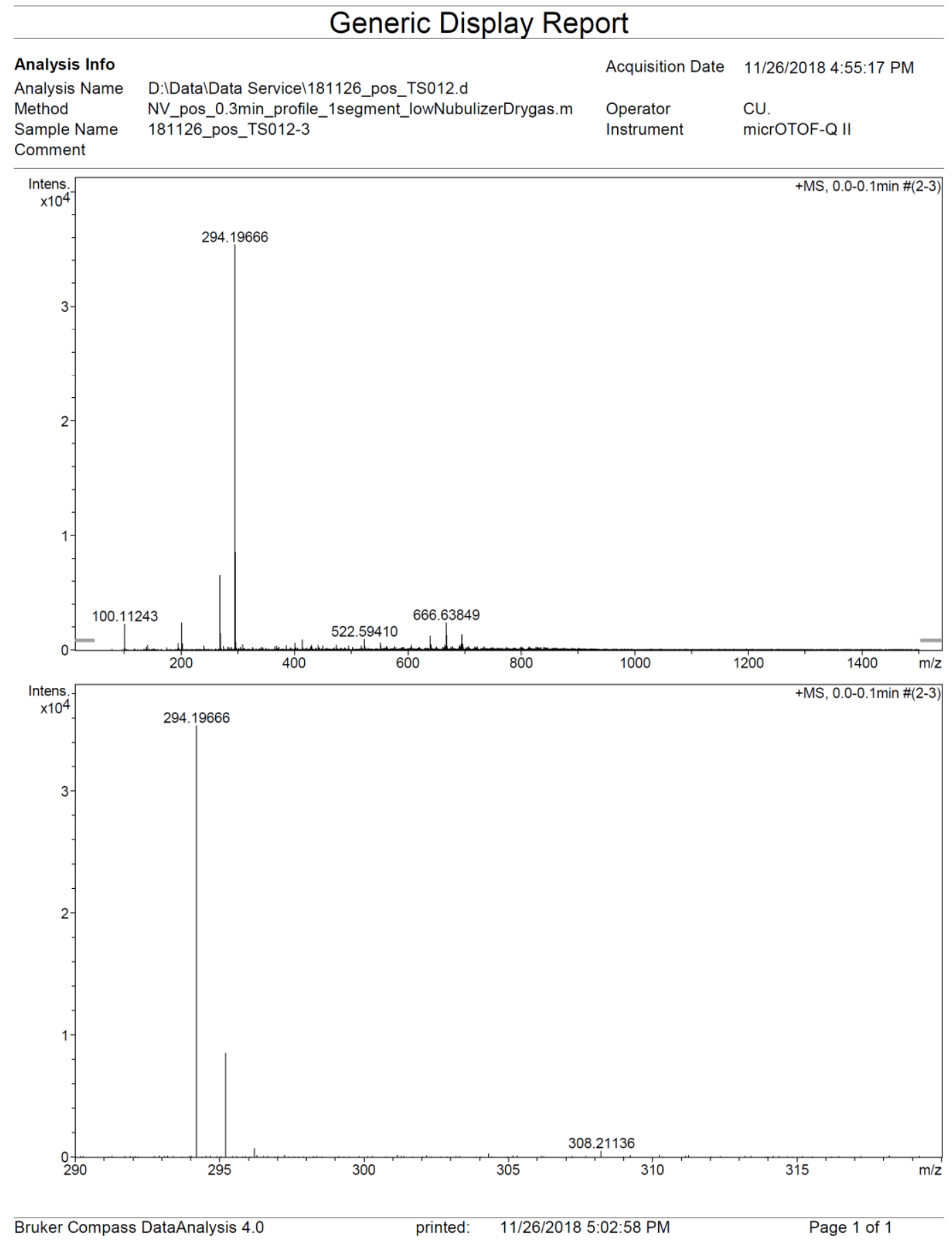

Figure S50. Mass spectrum of $\mathbf{2 h}$ 


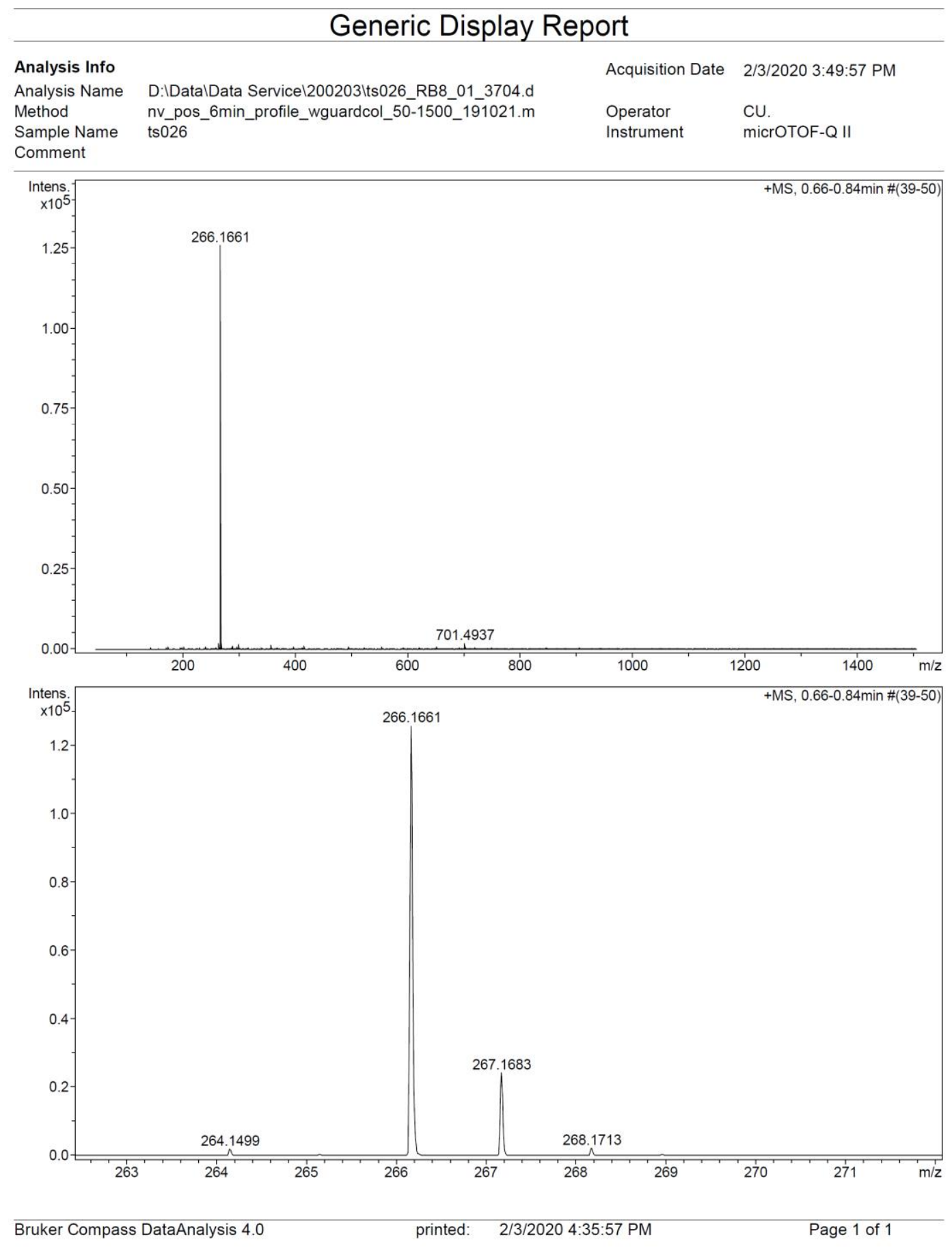

Figure S51. Mass spectrum of $\mathbf{2 i}$ 


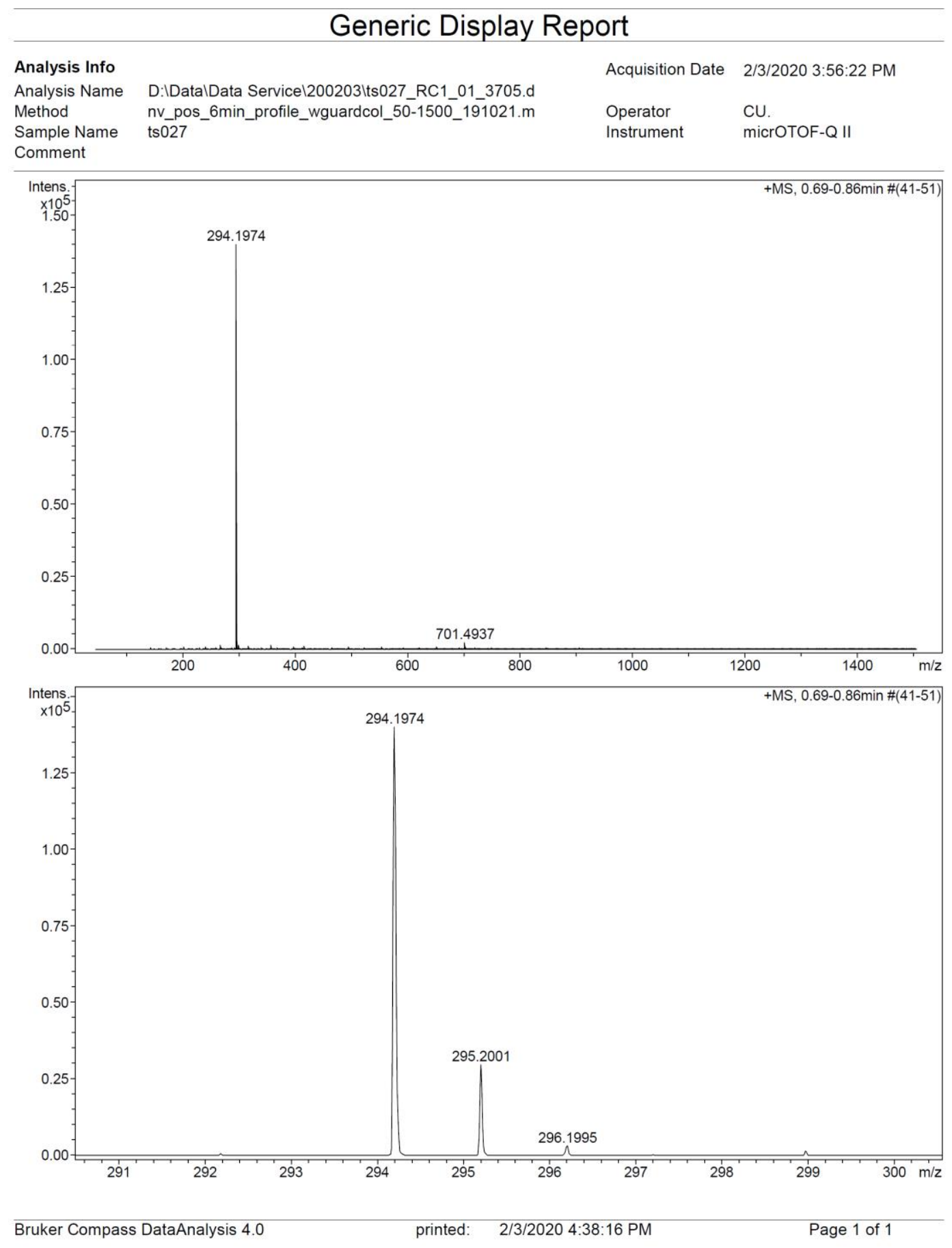

Figure S52. Mass spectrum of $\mathbf{2} \mathbf{j}$ 


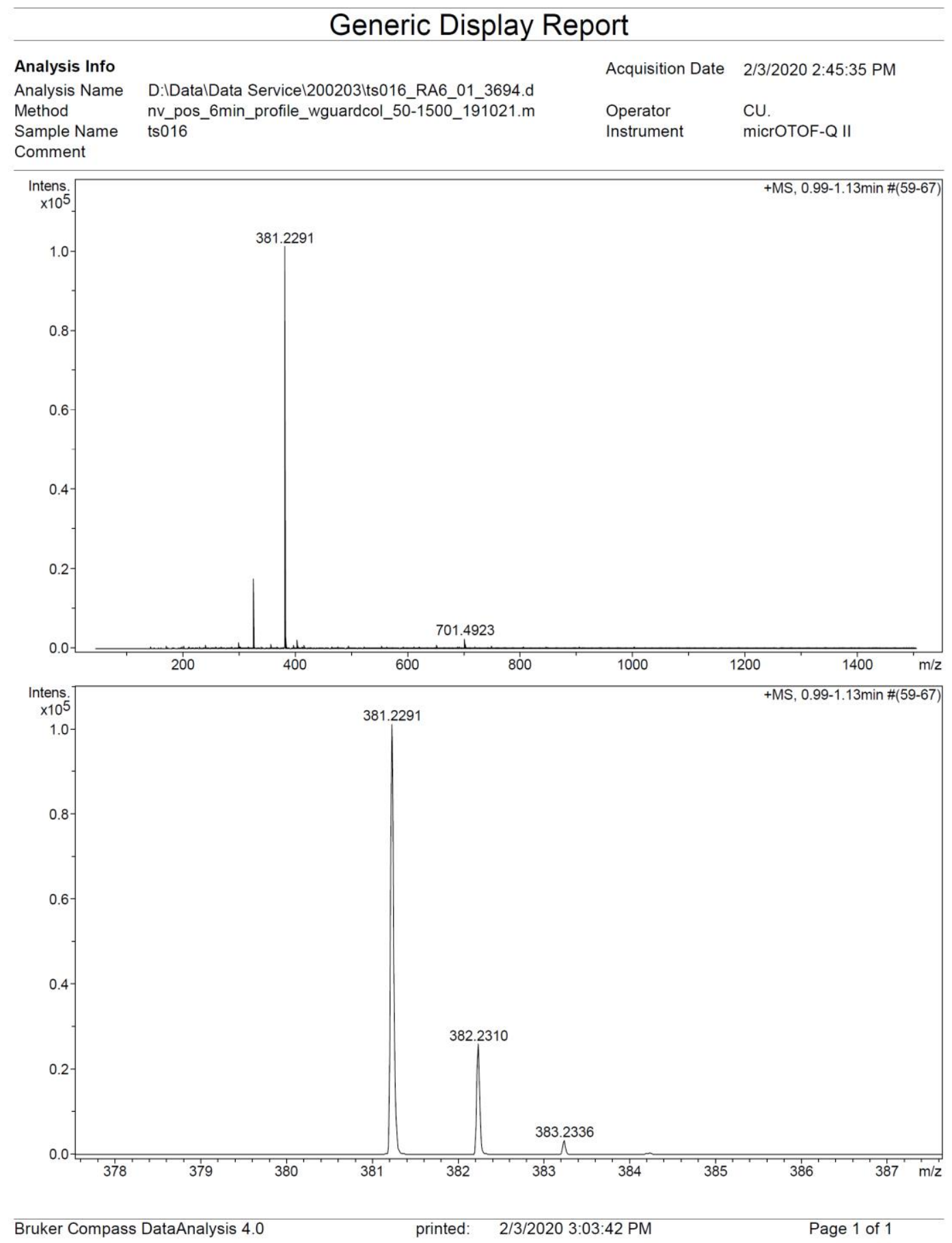

Figure S53. Mass spectrum of $2 \mathbf{k}$ 


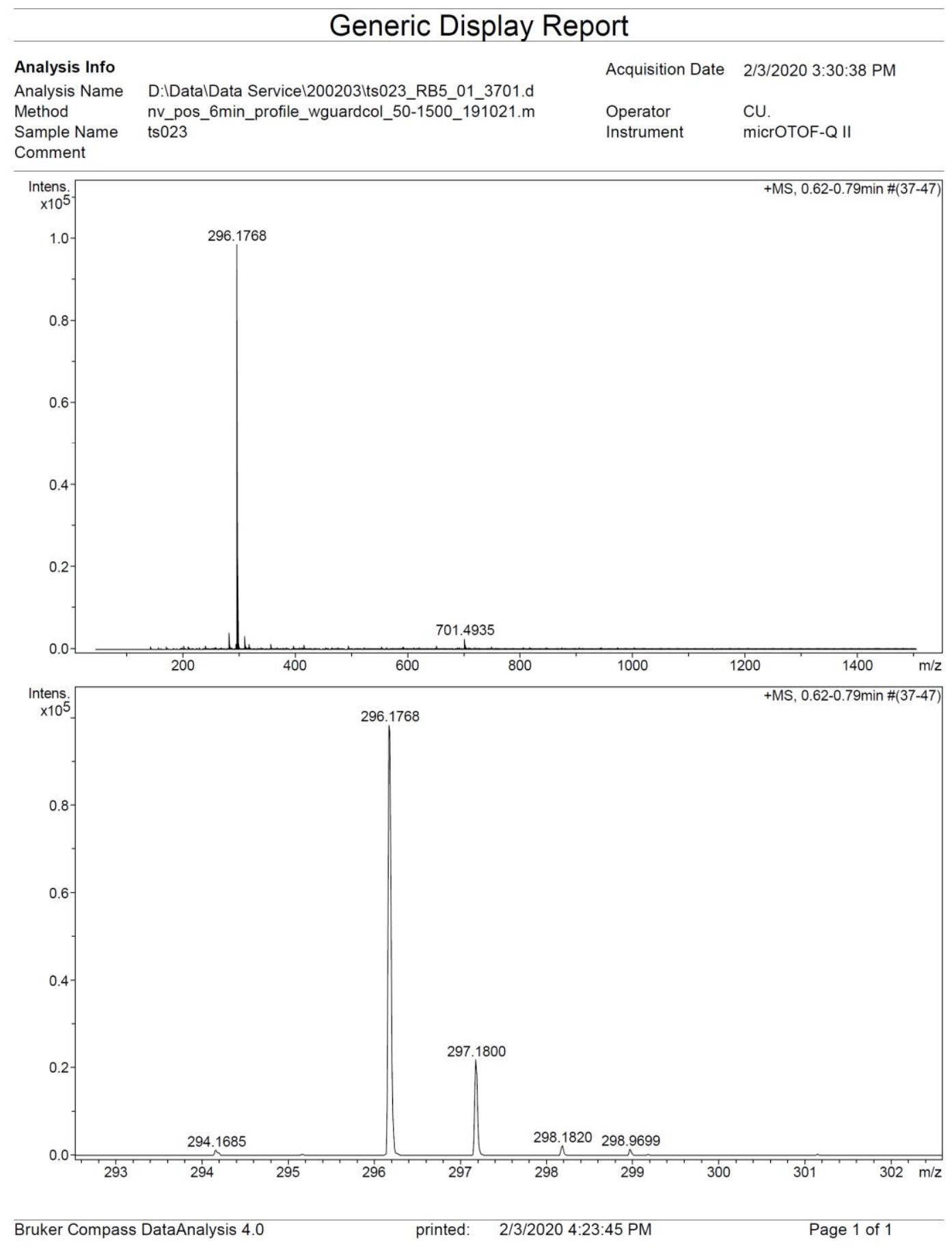

Figure S54. Mass spectrum of $\mathbf{3 b}$ 


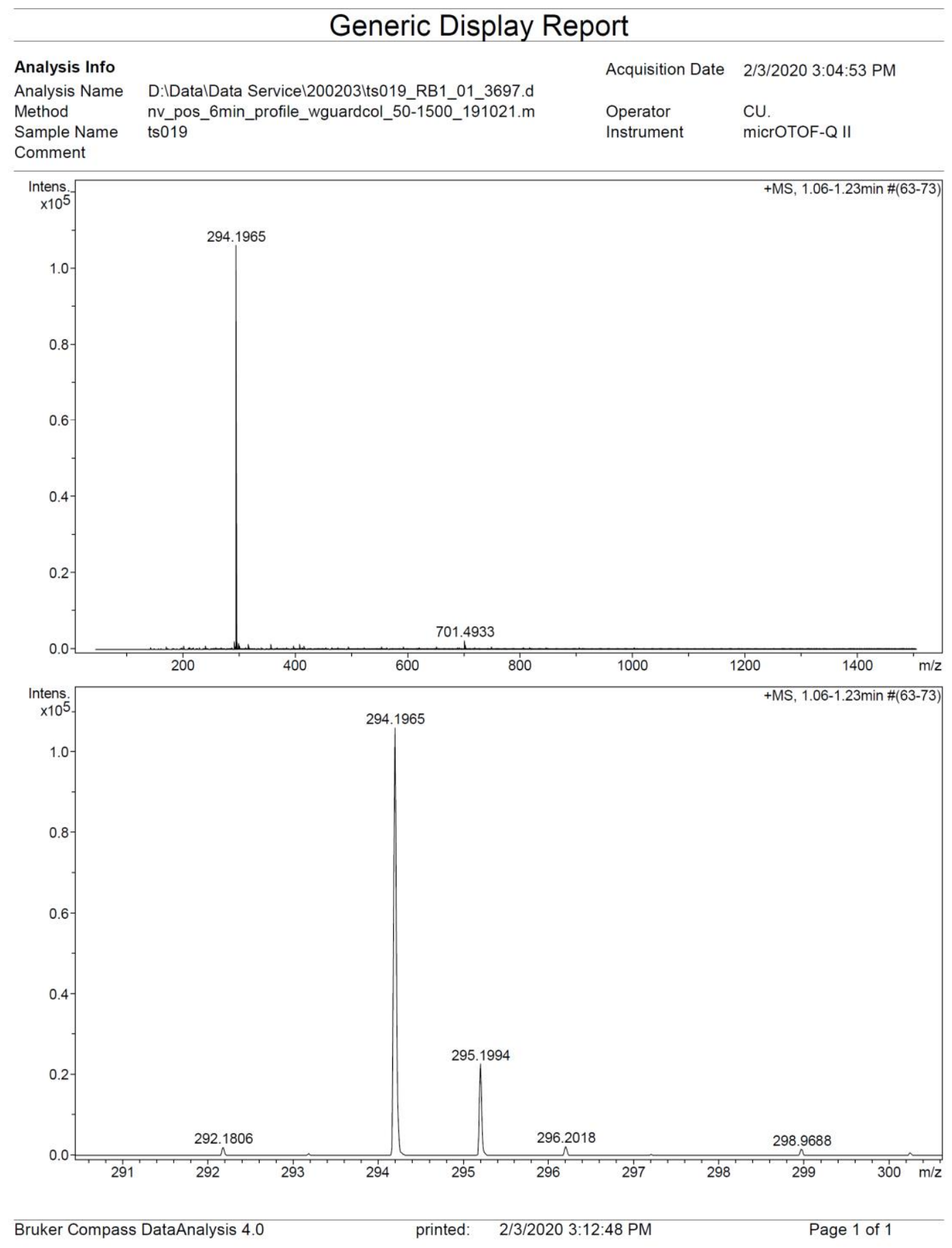

Figure S55. Mass spectrum of 3c 


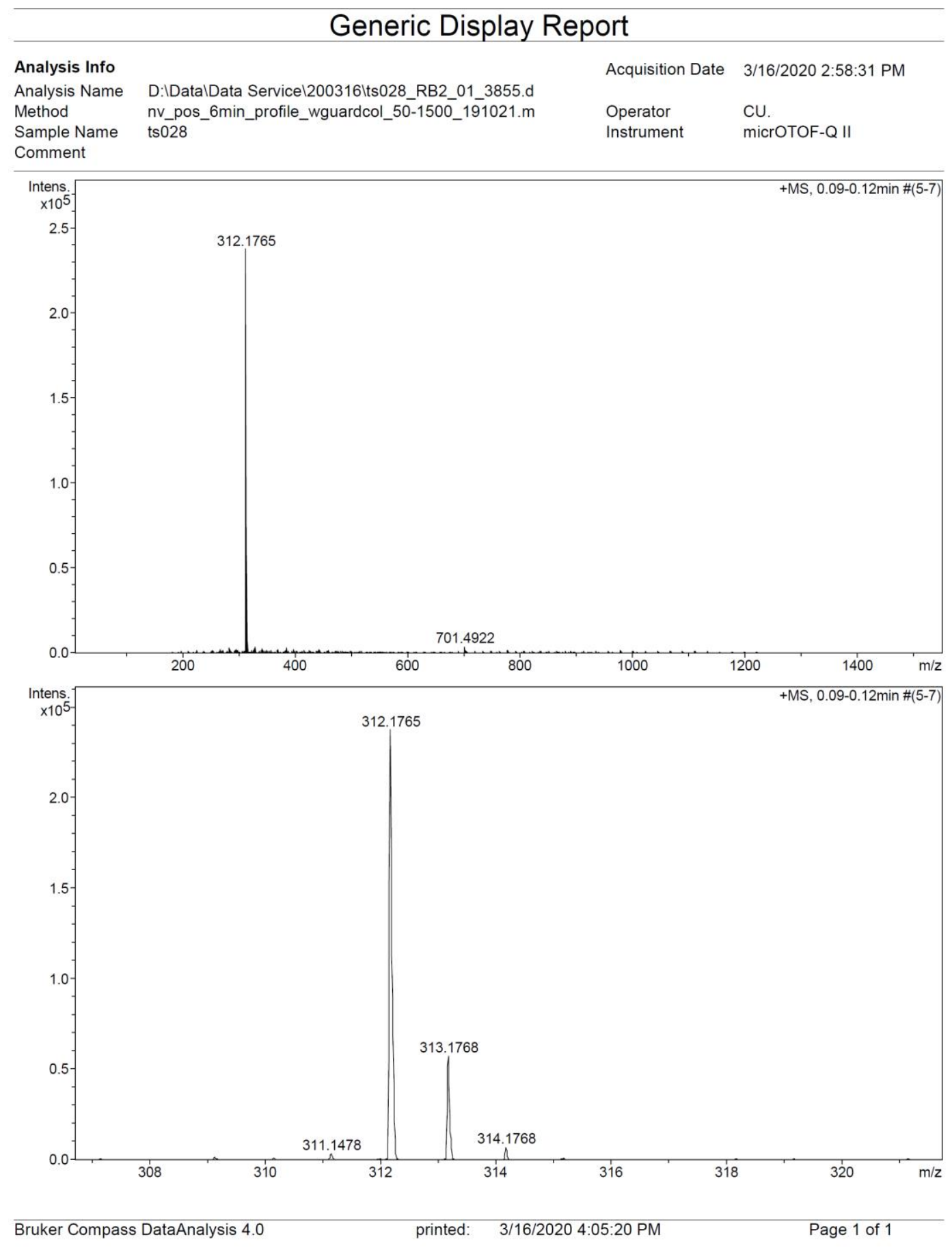

Figure S56. Mass spectrum of $\mathbf{3 d}$ 


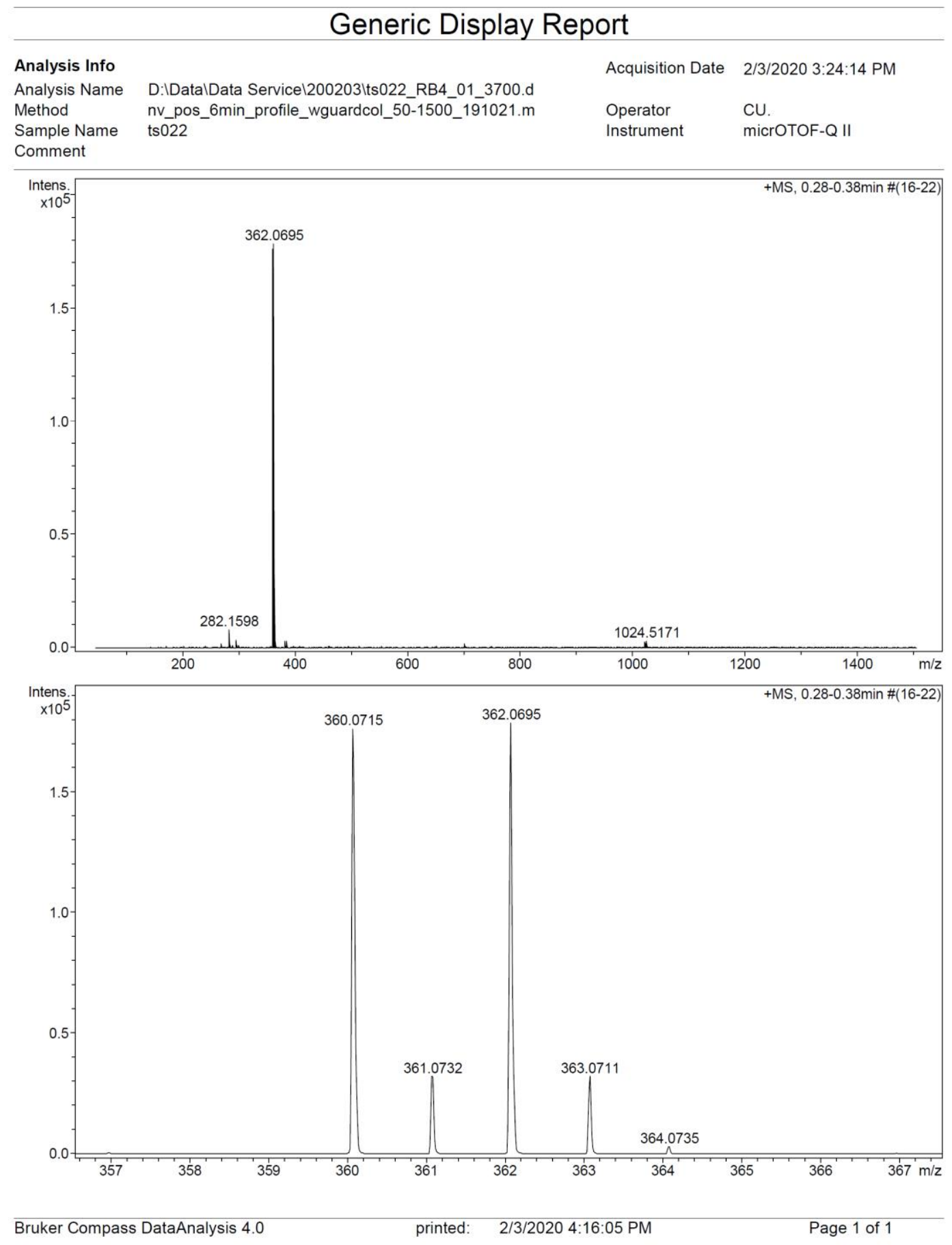

Figure S57. Mass spectrum of $\mathbf{3 e}$ 


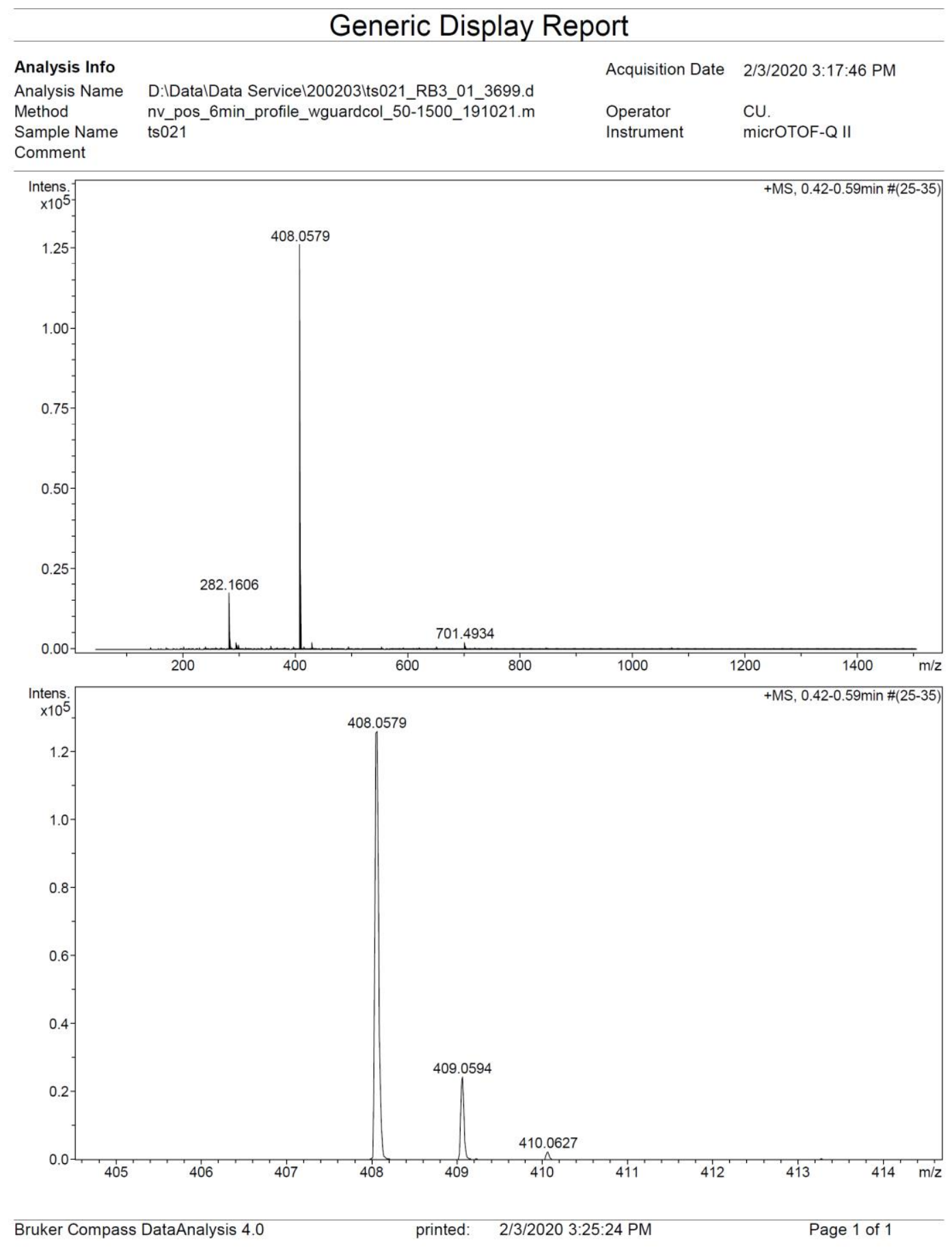

Figure S58. Mass spectrum of $\mathbf{3 f}$ 


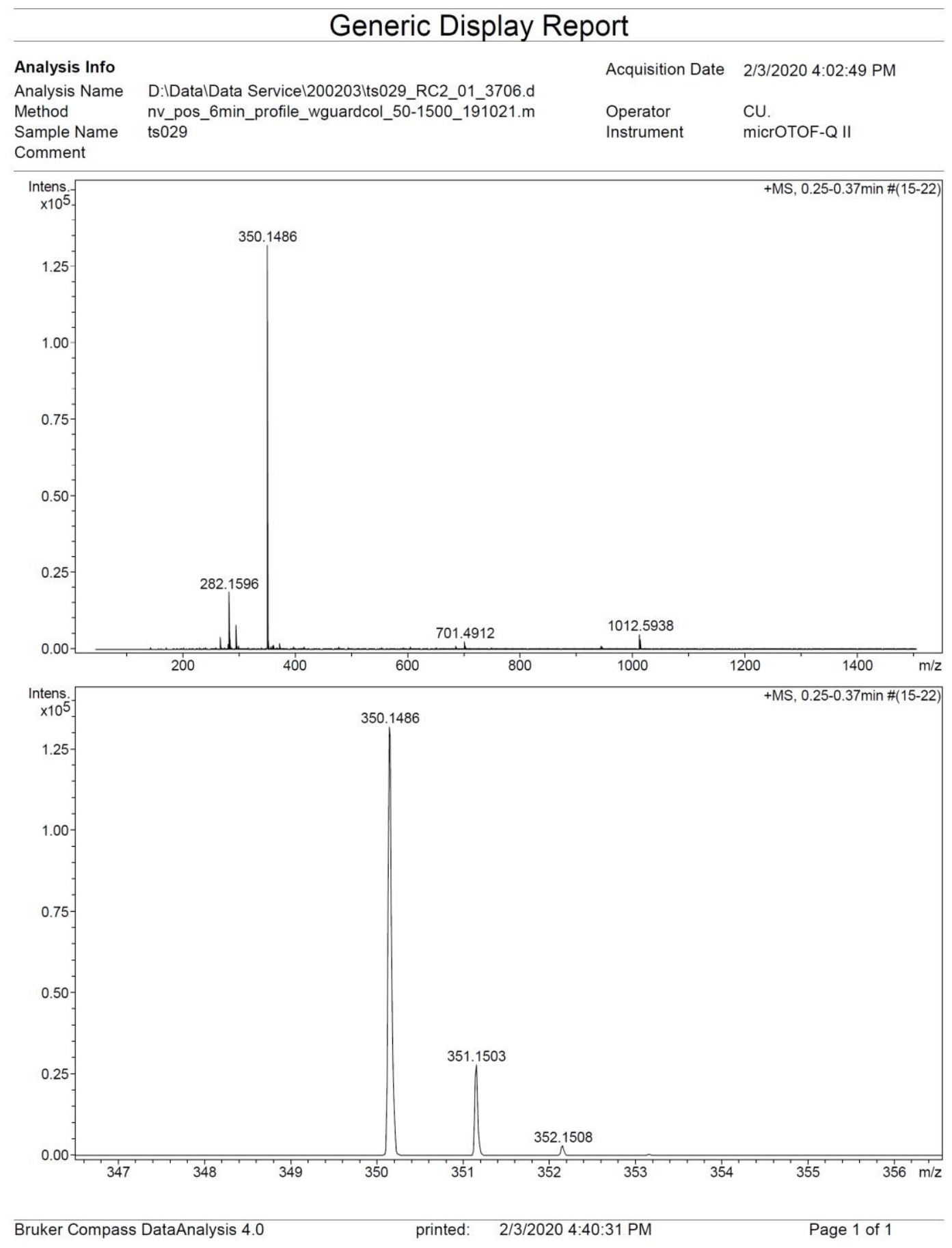

Figure S59. Mass spectrum of $\mathbf{3 g}$ 


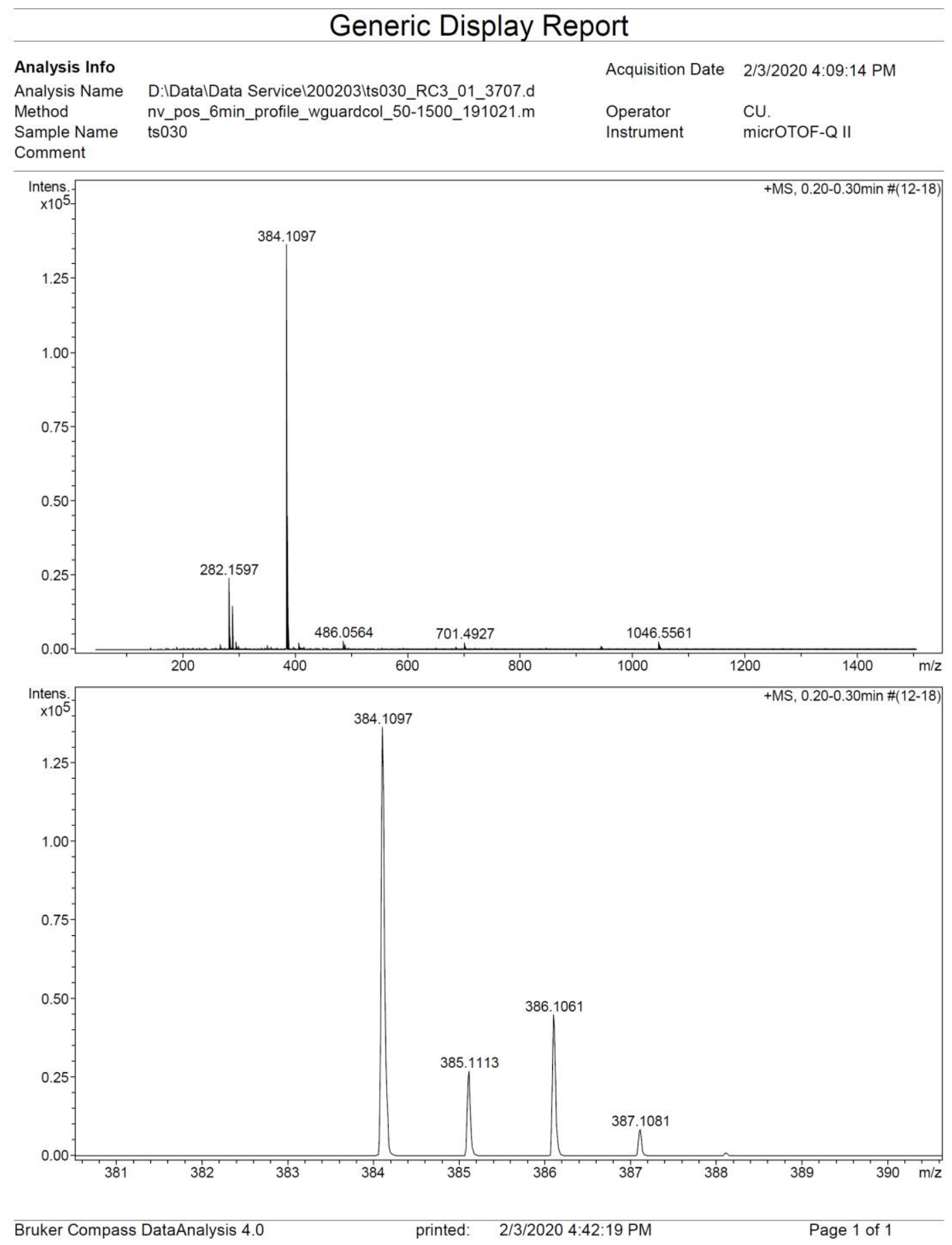

Figure S60. Mass spectrum of $\mathbf{3 h}$ 


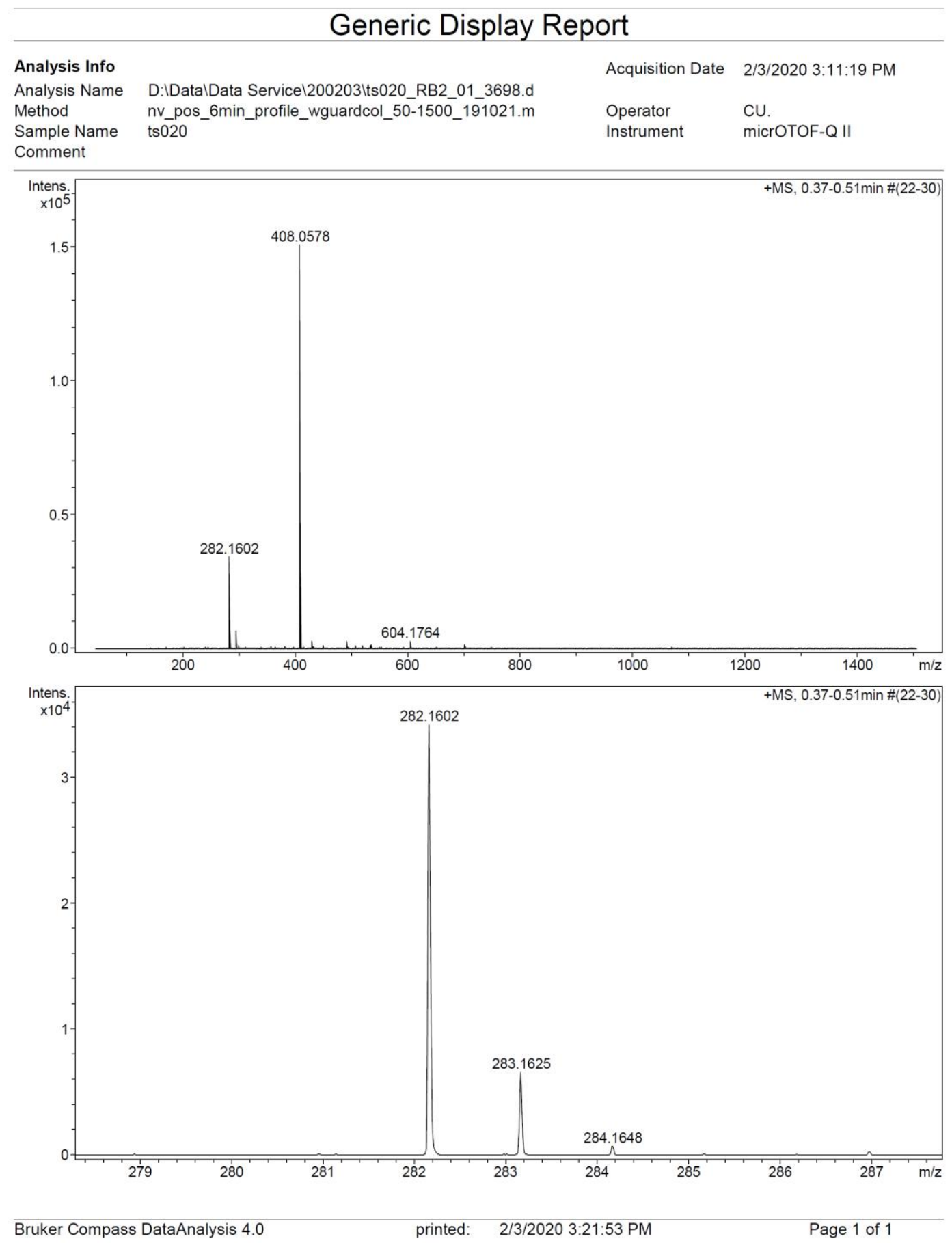

Figure S61. Mass spectrum of $\mathbf{3 i}$ 


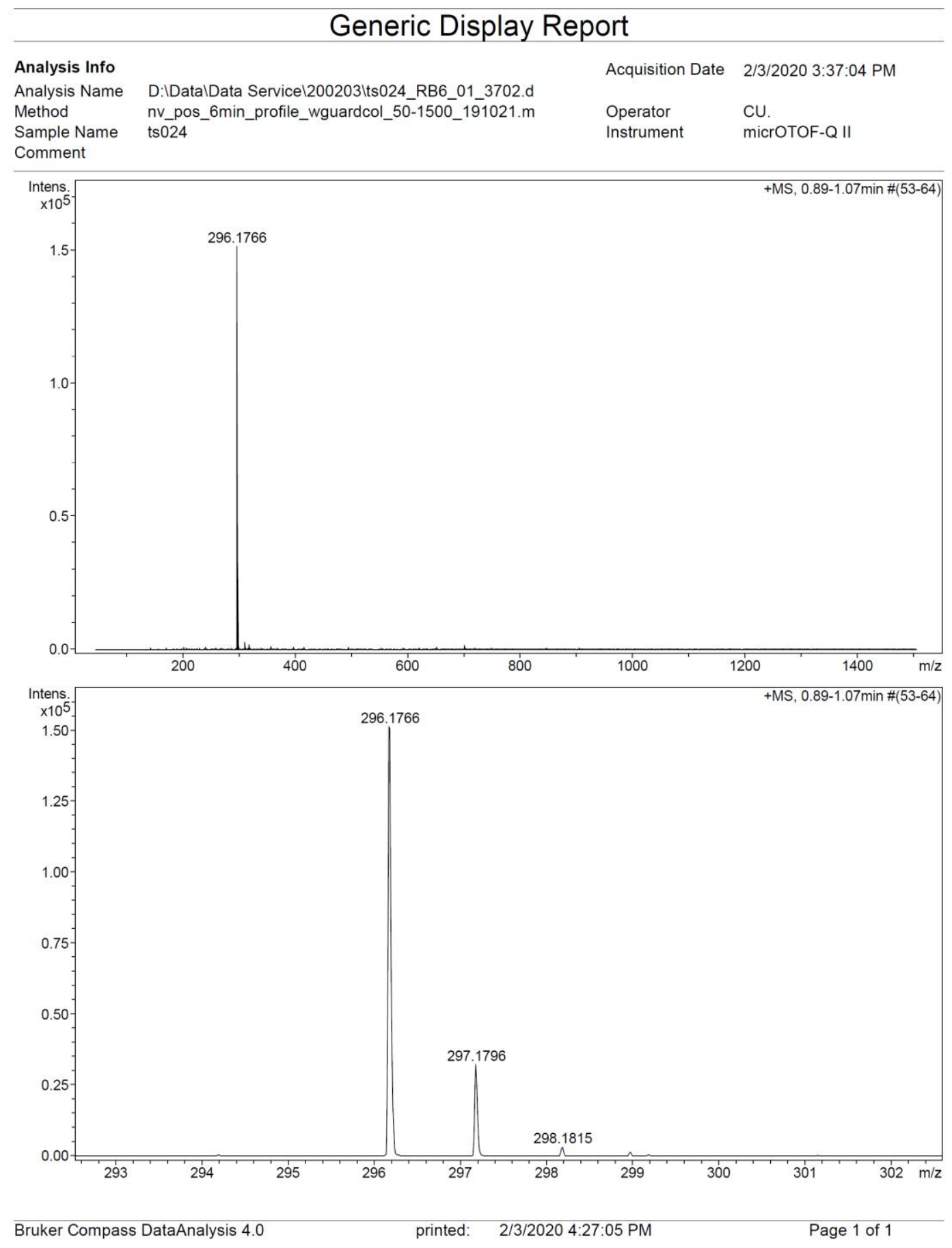

Figure S62. Mass spectrum of $\mathbf{3 j}$ 

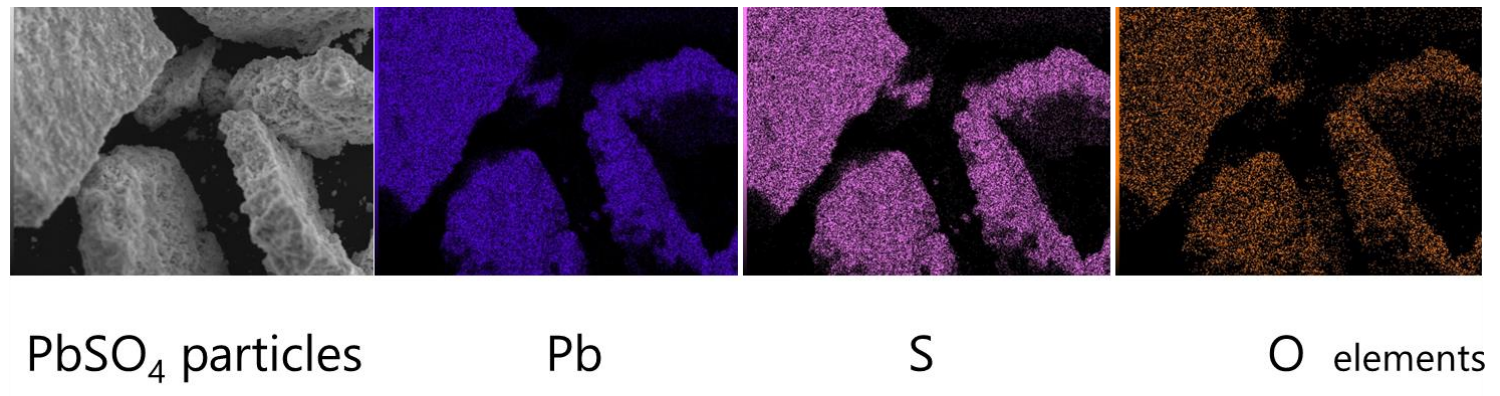

SEM image

EDX images

Figure S63. SEM and EDX images of $\mathrm{PbSO}_{4}$ particles

$\begin{array}{|lllllll|}\text { Formula mass\% } & \text { Atom\% } & \text { Sigma } & \text { Net } & \text { K ratio Line } & \\ \text { C } & 4.52 & 23.93 & 0 & 102354 & 0.0187257 & \mathrm{~K} \\ \mathrm{O} & 9.26 & 36.74 & 0.01 & 270588 & 0.1681221 & \mathrm{~K} \\ \mathrm{~S} & 4.74 & 9.39 & 0.01 & 792998 & 0.2136071 & \mathrm{~K} \\ \mathrm{As} & 9.19 & 7.79 & 0.13 & 141258 & 0.4097198 & \mathrm{~K} \\ \mathrm{~Pb} & 72.29 & 22.16 & 0.07 & 5357559 & 2.6174624 & \mathrm{M} \\ \text { Total } & 100 & 100 & & & & \end{array}$

Table S1. Quantitative analysis of $\mathrm{PbSO}_{4}$ particles by SEM/EDX 


\section{Reference}

[1] K. Ramadas, N. Janarthanan, R. Pritha, Synlett 1997, 1053-1054.

[2] K. Ramadas, N. Srinivasan, Tetrahedron Lett. 1995, 36, 2841.

[3] P. S. Dangate, K. G. Akamanchi, Tetrahedron Lett. 2012, 53, 6765-6767.

[4] L. Zhelyazkov, V. Baeva, Tr. Nauchnoizsled. Khim.-Farm. Inst. 1972, 7, 69-78.

[5] P. J. Bailey, K. J. Grant, L. A. Mitchell, S. Pace, A. Parkin, S. Parsons, Dalton 2000, 18871891.

[6] F. A. Cotton, L. M. Daniels, P. Huang, C. A. Murillo, Inorg. Chem. 2002, 41, 317-320.

[7] S. Cunha, M. T. Rodrigues, Tetrahedron Lett. 2006, 47, 6955-6956.

[8] M. Baeten, B. U. W. Maes, Adv. Synth. Catal. 2016, 358, 826-833. 